FERMILAB-TM-2553-E-PPD

\title{
Data Formatter Design Specification
}

\author{
Jamieson Olsen ${ }^{1}$, Tiehui Ted Liu ${ }^{1}$, Yasuyuki Okumura ${ }^{1,2}$ \\ ${ }^{1}$ Fermi National Accelerator Laboratory, Batavia, Illinois 60510, USA \\ ${ }^{2}$ University of Chicago, Chicago, Illinois 60637, USA
}

March 20, 2013

\begin{abstract}
The Fast TracKer (FTK) processor is an upgrade which adds a hardware-based tracking system to the ATLAS level-2 trigger system for high luminosity operation of the Large Hadron Collider (LHC), aimed for instantaneous luminosity of $3 \times 10^{34} \mathrm{~cm}^{-2} \mathrm{~s}^{-1}$ with 75 overlapping events per bunch crossing of $40 \mathrm{MHz}$. The FTK system will reconstruct tracks using data from the inner Pixel and SCT silicon detector at input rate up to $100 \mathrm{kHz}$ of level-1 trigger rate. In order to sustain the high input rate it is necessary to organize FTK as a set of independent engines, each working on a different region of the silicon tracker. The FTK system requires a layer of hardware to remap the ATLAS inner detector geometry to match the FTK $\eta-\phi$ towers. This hardware layer is the Data Formatter system. The Data Formatter system also performs clustering and data sharing for overlapping of neighboring $\eta$ - $\phi$ towers to avoid inefficiency due to the finite size of the beam luminous regions in $z$ coordinate and variety curvature of tracks. Based on the current design requirement and the need for future expansion capability, a full mesh Advanced Telecom Computing Architecture (ATCA) backplane interconnect is found to be a natural fit for the Data Formatter design. Our baseline design also works well as a general purpose FPGA-based processor board. The Data Formatter may prove useful in scalable systems where highly flexible, non-blocking, high bandwidth board to board communication is required. This specification note is aimed to present an overview of the current Data Formatter system design and show the data-driven bandwidth requirement study to support the current design.
\end{abstract}




\section{Contents}

1 Introduction $\quad 1$

2 The Inner Tracking Detector $\quad 1$

2.1 Layout of Pixel and SCT detectors . . . . . . . . . . . . . . . . . . 1

2.2 Readout Chains of Pixel and SCT detector . . . . . . . . . . . . . . 2

2.3 Expansion with Insertable B-Layer (IBL) . . . . . . . . . . . . . . . 2

3 The Fast Tracker and Data Formatter System 3

3.1 Overview of the Fast TracKer system and FTK tower structure . . . . . . . . . 3

3.2 The Data Formatter System . . . . . . . . . . . . . . . . . . . . 3

4 The Data Formatter System $\quad 4$

4.1 Conceptual Board-Level Design . . . . . . . . . . . . . . . . . 5

4.2 Data Sharing Paths . . . . . . . . . . . . . . . . . . 5

4.3 System-Level Design of Data Formatter System . . . . . . . . . . . . . . . . . 5

4.4 Input and Output Flexibility . . . . . . . . . . . . . . 6

5 Data Formatter Board $\quad \mathbf{8}$

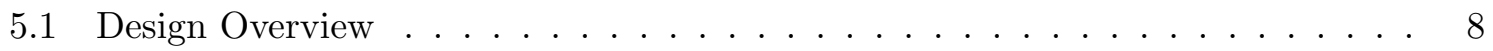

5.2 Core Processing Engines (FPGAs) $\ldots \ldots \ldots \ldots \ldots$

5.2 .1 Mezzanine Card Interface . . . . . . . . . . . . . . . . . . 9

5.2 .2 Fabric Interface . . . . . . . . . . . . . . . . . . . . 10

5.2 .3 Inter-FPGA link on board (Local Bus) . . . . . . . . . . . . . . 10

5.2 .4 RTM Interface . . . . . . . . . . . . . . . . . . . . . 10

5.2 .5 DDR3 Memory . . . . . . . . . . . . . . . . 10

5.3 Microcontroller . . . . . . . . . . . . . . . . . . . . 10

5.3 .1 IPMI Controller . . . . . . . . . . . . . . . . . . 11

5.3 .2 Ethernet Interface . . . . . . . . . . . . . . . . . . . . 12

5.3 .3 Flash Memory . . . . . . . . . . . . . . . . . . . 12

5.3 .4 FPGA Interface . . . . . . . . . . . . . . . . . . 12

5.3.5 Board Sensors and RTM Management . . . . . . . . . . . . . 13

5.3 .6 USB Serial Port . . . . . . . . . . . . . . . . . 13

5.3 .7 Microcontroller Software . . . . . . . . . . . . . . . . . 13

5.4 Clock Distribution . . . . . . . . . . . . . . . . . . . . . 13

5.4 Local Oscillators . . . . . . . . . . . . . . . . . . . . . 13

5.4 Backplane Clocks . . . . . . . . . . . . . . . . 13

5.5 Power Distribution . . . . . . . . . . . . . . . . . . . . 14

5.5 .1 Power Distribution Hierarchy . . . . . . . . . . . . . . . . 14

5.5 .2 Power Control Sequence . . . . . . . . . . . . . . . . . . . 14

5.6 Prototype Board . . . . . . . . . . . . . . . . . . 15

6 Mezzanine Cards $\quad 16$

6.1 Introduction . . . . . . . . . . . . . . . . . 16

6.2 FMC Connector . . . . . . . . . . . . . . . . . . . 17

6.3 Signal Levels and Bandwidth . . . . . . . . . . . . . . . . . . 17

6.4 Mezzanine Card Dimensions . . . . . . . . . . . . . . . . . . . 17

6.5 Power . . . . . . . . . . . . . . . . . . . . 18

6.6 Management and JTAG Interfaces . . . . . . . . . . . . . . . . . 18

6.7 Clocks . . . . . . . . . . . . . . . . . . 18 
6.8 Cluster Finder Mezzanine Card . . . . . . . . . . . . . . . . . . . . . . . 18

6.9 Prototype mezzanine card . . . . . . . . . . . . . . . . . . 19

7 Rear Transition Module $\quad 20$

7.1 Transceiver Modules . . . . . . . . . . . . . . . . . . 20

7.2 Mechanical Dimensions . . . . . . . . . . . . . . . . 20

7.3 RTM Power . . . . . . . . . . . . . . . . . . . . . . 21

7.4 Management Interface . . . . . . . . . . . . . . . . . . . . 21

7.5 Channel Assignments . . . . . . . . . . . . . . . . . . . . . 21

7.6 Prototype Board . . . . . . . . . . . . . . . . . 21

8 Bandwidth Requirements $\quad 22$

8.1 Hardware Configuration . . . . . . . . . . . . . . . . . . . 22

8.1.1 ROD - FPGA - FTK Tower Assignment . . . . . . . . . . . . . . . . 22

8.1 .2 Inter-Shelf Communication . . . . . . . . . . . . . . 24

8.2 Data Analysis . . . . . . . . . . . . . . . . . . . . . . 24

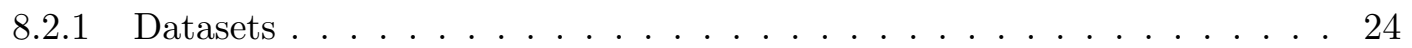

8.2.2 Number of Hits $\left(N_{\text {Pixel }}\right.$ and $\left.N_{\text {Strip }}\right) \ldots \ldots \ldots \ldots \ldots . \ldots \ldots$

8.2 .3 Input Data Volume. . . . . . . . . . . . . . . . . 25

8.2 .4 Output Data Volume . . . . . . . . . . . . . . . . . . 27

8.2 .5 Data Flow Routing Rules . . . . . . . . . . . . . . . . . 28

8.2 .6 Expected Number of Hits . . . . . . . . . . . . . . . . . . . . . . 29

8.2 .7 Bandwidth Requirements . . . . . . . . . . . . . . . . . 29

8.2.8 Extrapolation to Target LHC Operating Conditions . . . . . . . . . . . 30

8.2 .9 Bandwidth Requirement Summary . . . . . . . . . . . . . . . . 32

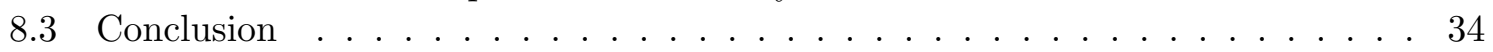

Appendix A System Analysis 35

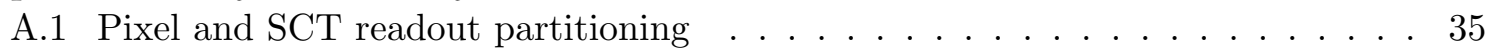

A.2 FTK $\eta-\phi$ Tower Partitioning . . . . . . . . . . . . . . . . . . . . . . . . 39

$\begin{array}{lll}\text { Appendix B AdvancedTCA Hardware Overview } & 40\end{array}$

B.1 Shelf . . . . . . . . . . . . . . . . . . . . . 40

B.2 Backplane . . . . . . . . . . . . . . . . . . . 40

B.3 Backplane Clocks . . . . . . . . . . . . . . . . . . . . . 41

B.4 Update Interface . . . . . . . . . . . . . . . . . . . . . . . . . . . . . . . . .

B.5 Intelligent Platform Management Interface . . . . . . . . . . . . . . . . . 41

B.6 Network Connectivity . . . . . . . . . . . . . . . . . . . . 42

B.7 Hub and System Controller Boards . . . . . . . . . . . . . . . 43

B.8 Power Supply . . . . . . . . . . . . . . . . . . . . . . . . . 43

$\begin{array}{lll}\text { Appendix C } & 44\end{array}$

C.1 FMC Pinout . . . . . . . . . . . . . . . . . . . . . . 44

C.2 FMC Connector . . . . . . . . . . . . . . . . . . 46

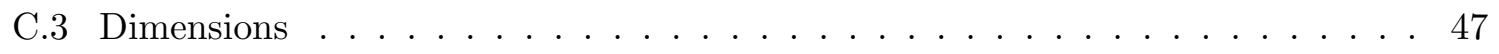

C.4 Bezel Detail . . . . . . . . . . . . . . . . . . 48

$\begin{array}{ll}\text { Appendix D RTM Details } & 49\end{array}$

D.1 Transceiver Numbering . . . . . . . . . . . . . . . . . . . . . . . 49

D.2 Transceiver Connector Port Assignments . . . . . . . . . . . . . . 50

D.3 Rear Panel Detail . . . . . . . . . . . . . . . . . . . . . . . . 52 
$\begin{array}{lll}\text { Appendix E Initial Firmware Study } & \mathbf{5 3}\end{array}$

E.1 Serial Transceivers . . . . . . . . . . . . . . . . . . . 53

E.2 Packet Switch Preliminaty Consideration . . . . . . . . . . . . . 53

$\begin{array}{lll}\text { Appendix F ROD IDs } & \mathbf{5 5}\end{array}$

F.1 132 Pixel RODs . . . . . . . . . . . . . . . . . . . 55

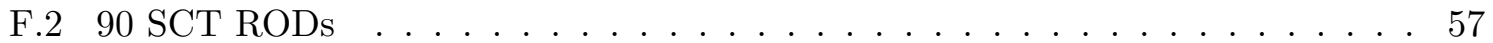

$\begin{array}{ll}\text { Appendix G FTK Tower ID } & 58\end{array}$

$\begin{array}{ll}\text { Appendix H SLINK - FPGA Assignments } & 59\end{array}$

$\begin{array}{lll}\text { Appendix I Pixel and SCT Data Format } & \mathbf{6 1}\end{array}$

I.1 S-Link Header and Trailer . . . . . . . . . . . . . . . . . . . 61

I.2 Pixel Raw Data . . . . . . . . . . . . . . . . . . . . . . . . . 62

I.3 SCT Raw Data . . . . . . . . . . . . . . . . . . . . 62

I.4 Number of SLINK Words . . . . . . . . . . . . . . . . . . . 63

$\begin{array}{lll}\text { Appendix J Tails in the Number of Hits per Module } & 65\end{array}$

$\begin{array}{ll}\text { Appendix K Downstream Data Flow } & 66\end{array}$

$\begin{array}{lll}\text { Appendix L } & \text { Data Flow Model } & \mathbf{7 0}\end{array}$

$\begin{array}{ll}\text { Appendix M Data Volume Luminosity Dependence } & 71\end{array}$

$\begin{array}{ll}\text { Appendix N Data Volume Monte Carlo Comparison } & 73\end{array}$

$\begin{array}{ll}\text { Appendix O Clustering } & \mathbf{7 5}\end{array}$

O.1 Output Data Volume . . . . . . . . . . . . . . . . . . . 75

O.2 Bandwidth Requirement . . . . . . . . . . . . . . . . . . 77

$\begin{array}{lll}\text { Appendix P } & \text { Unconstrained Data Volume Study } & \mathbf{7 8}\end{array}$

P.1 Data Sharing . . . . . . . . . . . . . . . . . . . . 78

P.2 Data Volume Study Results . . . . . . . . . . . . . . . . . . 79 


\section{List of Figures}

1 ATLAS Inner Detector Modules . . . . . . . . . . . . . . . . . . 2

2 FTK $\phi-\eta$ segmentation . . . . . . . . . . . . . . . . . . . 4

3 Data Formatter System . . . . . . . . . . . . . . . . . 5

4 Data Formatter conceptual board design . . . . . . . . . . . . . . . 6

5 Internal communication among the 64 FPGAs . . . . . . . . . . . . . 7

6 A 3 D representation of FPGA interconnects. . . . . . . . . . . . . 7

7 Data Formatter block diagram f . . . . . . . . . . . . . . 8

8 Data Formatter Board 3D layout . . . . . . . . . . . . . . . . . . 9

$9 \quad$ IPMC Microcontroller . . . . . . . . . . . . . . . . . . . . . . . 11

10 A typical shelf configuration . . . . . . . . . . . . . . . . . 12

11 Power distribution . . . . . . . . . . . . . . . . . . . . 14

12 Prototype Data Formatter board . . . . . . . . . . . . . . . . . 15

13 A mezzanine test card . . . . . . . . . . . . . . . . . . . 16

14 Rear Transition Module . . . . . . . . . . . . . . . . . . . . . . 20

15 Module sharing between FPGAs . . . . . . . . . . . . . . . . 23

16 Inter-shelf communication . . . . . . . . . . . . . . . . . . 24

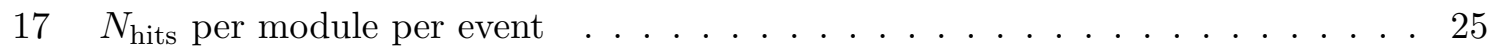

18 Number of hits per ROD . . . . . . . . . . . . . . . . . . 26

19 Summary of $N_{\text {hits }}$ to individual 64 FTK towers . . . . . . . . . . . . . . 27

20 Summary of $N_{\text {hits }}$ to individual 64 FTK towers, Pixel and SCT. . . . . . . . . 27

21 Summary of $N_{\text {hits }}$ to individual 64 AUXs and SSBs. . . . . . . . . . . . . . 28

22 Summary of $N_{\text {Pixel }}$ and $N_{\mathrm{SCT}}$ transferred in the DF system. . . . . . . . . 30

23 Input data format . . . . . . . . . . . . . . . . . . . 30

24 Summary of $N_{\text {words }}$ to individual 64 AUXs and SSBs. . . . . . . . . . . . 31

25 Summary of $N_{\text {words }}$ transferred in the DF system. . . . . . . . . . . . . . 31

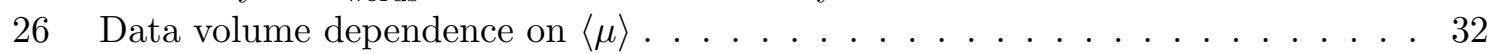

27 Number of Pixel hits as a function of $\langle\mu\rangle \ldots \ldots \ldots \ldots \ldots$

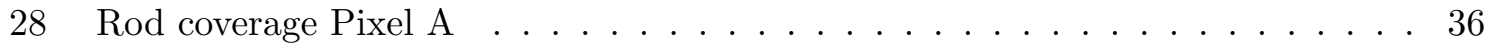

29 ROD coverage Pixel C . . . . . . . . . . . . . . . . 36

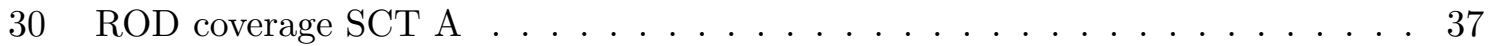

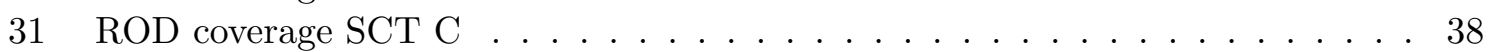

32 FTK tower $\eta$ boundaries . . . . . . . . . . . . . . . . . . . . . . 39

33 ATCA board and shelf . . . . . . . . . . . . . . . . . 40

34 Shelf Manager board . . . . . . . . . . . . . . . . . . . . . . 41

35 ATCA backplane connections . . . . . . . . . . . . . . . 43

36 ATCA system controller board . . . . . . . . . . . . . . . . . . . 44

37 FMC connector . . . . . . . . . . . . . . . . . . . . . 46

38 Mezzanine Card dimensions . . . . . . . . . . . . . . . . . . . 47

39 Mezzanine card and bezel location . . . . . . . . . . . . . . . . . 48

40 RTM transceivers . . . . . . . . . . . . . . . . . . . . . . . 49

$41 \quad$ Rear Panel Detail . . . . . . . . . . . . . . . . . . . . . . . . 52

42 Routing firmware overview . . . . . . . . . . . . . . . . . 54

43 Banyan network switch . . . . . . . . . . . . . . . . . . . . . . . . . . . . . 54

44 ROD event header . . . . . . . . . . . . . . . . . . . 61

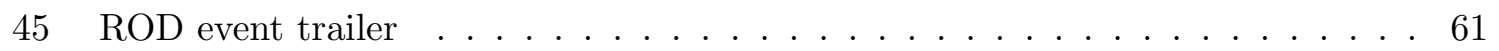

46 Pixel raw data . . . . . . . . . . . . . . . . . . . . . 62

47 Header words in Pixel output. . . . . . . . . . . . . . . . . . 62

48 SCT raw data . . . . . . . . . . . . . . . . . . . 63 
49 Header words in SCT output . . . . . . . . . . . . . . . . . . 63

50 SLINK words for Pixel RODs . . . . . . . . . . . . . . . . . . 63

51 SLINK words for for SCT RODs . . . . . . . . . . . . . . . . 64

$52 N_{\text {hits }}$ per module per event distribution $(\log$ scale $) \ldots \ldots \ldots \ldots$

53 Data sharing flowchart . . . . . . . . . . . . . . . . . 70

54 Pixel data volume dependence on pileup. . . . . . . . . . . . . . . 71

55 SCT data volume dependence on pileup. . . . . . . . . . . . . . . . . 72

56 Number of Pixel hits (barrel) _ . . . . . . . . . . . . . . . . . . 72

57 Number of SCT hits (barrel) $\ldots \ldots \ldots \ldots \ldots \ldots$. . . . . . . . . . . . 72

58 Pixel and SCT hits as a function of $\langle\mu\rangle \ldots \ldots \ldots \ldots \ldots$

59 Pixel and SCT hits as a function of $\langle\mu\rangle$, barrel layers . . . . . . . . . . . . 74

60 Module sharing between FPGAs . . . . . . . . . . . . . . . . 78

61 Summary of $N_{\text {hits }}$ and bandwidth, no module-ROD constraints. . . . . . . . . 79

$62 N_{\text {words }}$ transferred, no module-ROD cabling constraints. . . . . . . . . . 80

\section{List of Tables}

1 Data Formatter input readout links . . . . . . . . . . . . . . . . . 3

$2 \quad$ FPGA assignments . . . . . . . . . . . . . . . . . 22

3 Data sample summary . . . . . . . . . . . . . . . . . . 25

4 Number of hits per module . . . . . . . . . . . . . . . . 26

5 Average number of hits per ROD per event . . . . . . . . . . . . 26

6 Summary of $N_{\text {hits }}$ sent to the downstream. . . . . . . . . . . . . . 28

7 Summary of $N_{\text {hits }}$ transferred in the DF system. . . . . . . . . . . . . . . . 29

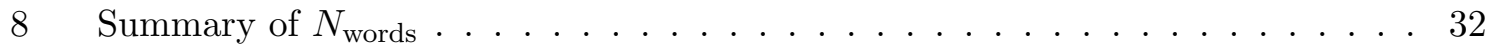

9 Summary of the expected $N_{\text {hits }}$ at the target LHC operating conditions. . . . . 33

$10 N_{\text {words }}$ and link bandwidth at target LHC operating conditions. . . . . . . . 34

11 Number of RODs for Pixel readout . . . . . . . . . . . . . . . . . . . . . . . . . . . . . 35

12 Number of RODs for SCT readout . . . . . . . . . . . . . . . . . 35

13 Pixel module count . . . . . . . . . . . . . . . . . . 36

14 SCT module count . . . . . . . . . . . . . . . . . . 36

15 FMC connector pinout . . . . . . . . . . . . . . . . . 45

16 RTM channel assignments J32/RP32 . . . . . . . . . . . . . . . . . . 50

17 RTM channel assignments J33/RP33 . . . . . . . . . . . . . . . . 51

18 FTK Tower ID and Tower Location. . . . . . . . . . . . . . . . . 58

19 ROD assignments for FTK towers C . . . . . . . . . . . . . . . 59

20 ROD assignment for FTK towers A . . . . . . . . . . . . . . . 60

21 Source IDs . . . . . . . . . . . . . . . . . . . . . . . . . 61

22 Tail components of the $N_{\text {hits }}$ per Pixel module . . . . . . . . . . . . . 65

23 Tail components of the $N_{\text {hits }}$ per SCT module per event . . . . . . . . . . 65

24 Output $N_{\text {hits }}$ to individual 32 FTK towers in C-Side. . . . . . . . . . . . 66

25 Output $N_{\text {hits }}$ to individual 32 FTK towers in A-Side. . . . . . . . . . . . . 67

26 Extrapolation of output $N_{\text {hits }}$ to individual 32 FTK towers in C-Side . . . . . . 68

27 Extrapolation of output $N_{\text {hits }}$ to individual 32 FTK towers in A-Side . . . . . . 69

28 Hits to AUX/SSB clustering $\mathrm{C}$ at $14 \mathrm{TeV}$ and $\langle\mu\rangle=70 \ldots \ldots$. . . . . . 75

29 Hits to AUX/SSB clustering A at $14 \mathrm{TeV}$ and $\langle\mu\rangle=70 \ldots \ldots$. . . . . 76

30 Expected $N_{\text {hits }}$ with clustering at high luminosity. . . . . . . . . . . . 77

$31 N_{\text {words }}$ and bandwidth requirements with clustering at high luminosity. . . . . . 77

$32 N_{\text {words }}$ and BW req. at $\sqrt{s}=14 \mathrm{TeV},\langle\mu\rangle=70.0$, no module-ROD constraints. 81 


\section{Introduction}

The Large Hadron Collider (LHC) at CERN will extend the frontiers of particle physics with its unprecedented high energy and luminosity. Inside the LHC, bunches with more than $10^{11}$ protons will collide every $25 \mathrm{~ns}$ to provide $14 \mathrm{TeV}$ proton-proton collisions at a design luminosity above $1 \times 10^{34} \mathrm{~cm}^{-2} \mathrm{~s}^{-1}$. The ATLAS (A Toroidal LHC ApparatuS) detector is a general purpose detector located at one of the collision points of the LHC to search new and very rare physics phenomena.

Since it is essential to increase the luminosity in order to conduct this type of study at ATLAS, improvement of the trigger system is necessary to enable reasonable reduction of the background online. The Fast TracKer (FTK) [3] is a trigger upgrade program that permits quick track reconstruction to provide the full track lists to the Level-2 algorithm with instantaneous luminosity of $3 \times 10^{34} \mathrm{~cm}^{-2} \mathrm{~s}^{-1}$ at a level-1 trigger rate up to $100 \mathrm{kHz}$. The FTK system is a massively parallel hardware-based processing engine for fast tracking based on inner Pixel and SCT silicon hits. In order to sustain the high input rate, it is necessary to organize FTK as a set of independent engines, each working on a different region of the silicon detector. Each FTK $\eta$ - $\phi$ tower region has its own "core processor" for a total 64 engines working independently. This segmentation generates some inefficiency at region boundaries due to the finite size of the beam's luminous region and the variety pf curvature for the low-momentum charged particle that must be removed by allowing a overlap region at the boundary.

The FTK system includes a Data Formatter system to remap the ATLAS inner detector geometry to match the FTK $\eta-\phi$ towers. The Data Formatter system also performs hitclustering and data sharing in overlap regions. Based on the current design requirements and the need for future expansion capabilities, a full mesh Advanced Telecom Computing Architecture (ATCA) backplane interconnect is found to be a natural solution for the Data Formatter design. We present the current design specification of the Data Formatter system, as well as the data-driven bandwidth requirement estimation study to support the design. This specification note consists of the following sections:

- ATLAS Inner Detector system (Section 2)

- Overview of Fast Tracker and concept of the Data Formatter system (Section 3)

- System-level design of the Data Formatter System (Section 4)

- Functions and design of the hardware components (Section 5, 6, 7)

- Data-driven bandwidth requirement estimation study (Section 8).

The early note of the Data Formatter hardware specification [1] is available to offer an historical look back at the early Data Formatter design process.

\section{The Inner Tracking Detector}

\subsection{Layout of Pixel and SCT detectors}

Figure 1 shows ATLAS Pixel and SCT silicon detectors. The Pixel detector is composed of three barrel layers (radius 50 to $123 \mathrm{~mm}$ ) and six end-cap disks (at $z=495 \mathrm{~mm}$ to $650 \mathrm{~mm}$ ). All of the 1,744 pixel modules are identical and consist of 46,080 readout channels (pixel sensor size is $400 \mu \mathrm{m} \times 50 \mu \mathrm{m}$ with thickness of $250 \mu \mathrm{m}$ ) per module for a total of 80.4 million readout channels. 


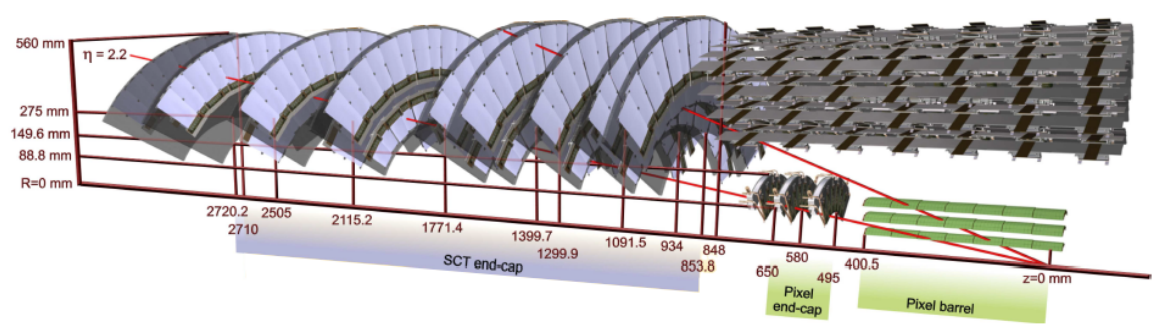

Figure 1: The ATLAS inner Pixel and SCT detector modules.

The SCT detector is composed of four barrel layers (radius 299 to $514 \mathrm{~mm}$ ) and six end-cap disks (at $z=853$ to $2720 \mathrm{~mm}$ ) to measure the charged particle positions with two stereo silicon strip layers. The SCT barrel module consists of 768 silicon strips $(63 \mathrm{~mm} \times$ 80 mum with thickness of $285 \mu \mathrm{m}$ ). Four SCT barrel layers are constructed from 2,112 sensors mounted to "stave" support structures. SCT end-cap modules are trapezoidal shaped and come in three varieties: inner modules measure $45 \mathrm{~mm}, 55 \mathrm{~mm}, 61 \mathrm{~mm}$ (inner width, outer width, length); middle modules measure $55 \mathrm{~mm}, 75 \mathrm{~mm}, 119 \mathrm{~mm}$; and outer modules measure $56 \mathrm{~mm}, 72 \mathrm{~mm}, 123 \mathrm{~mm}$. A total of 1,976 modules are used to construct the SCT end-cap disks.

\subsection{Readout Chains of Pixel and SCT detector}

Inner detector front end electronics are implemented in radiation-hardened application-specific integrated circuit (ASIC) devices which are mounted on the modules. These front end ASICs interface the silicon sensors and incorporate analog circuitry to amplify the signals and compare the signal level against a programmable threshold. Pixel ASICs store the "hit" pixel coordinate as well as time stamps (BCID, L1ID) and amplitude (time over threshold) in a buffer, which is read out following L1 trigger accept signal. The SCT ASICs store the "hit" strip address as well as time stamps and 3-bit timing information for previous, current, and next bunch crossing hit information. The data format and the contained information are summarized in Appendix I.

Chains of front end ASICs are connected over fiber optic links to the Readout Driver (ROD) electronics, which are located off-detector in USA15 in the ATLAS cavern. RODs receive serialized data from the detector after a L1 trigger accept and are responsible for de-serializing the data, error checking, local event building and data monitoring tasks. Each ROD services between 6 and 26 fiber input from pixel modules or up to 96 fiber inputs from SCT modules. Table 1 summarizes the number of modules and readout links for pixel and SCT detectors. The current system makes use of 222 fiber optic SLINK [16] cables which send the data to the Read-Out System (ROS) located downstream in the ATLAS DAQ system. Appendix A summarizes the details of the partitioning of the Pixel and SCT readout (module-ROD links). The number of readout links is planned to be increased to maintain the bandwidth for the higher hit occupancy after scheduled shutdown from 2013.

\subsection{Expansion with Insertable B-Layer (IBL)}

Plans are currently underway to install an "insertable B-layer" (IBL) pixel detector during the scheduled shutdown from 2013. The IBL consists of additional pixel modules arranged in a barrel layer near the beam pipe at a radius of approximately $34 \mathrm{~mm}$. A total of 224 modules 


\begin{tabular}{|l|l|l|l|}
\hline Sub-detector & Partition & Modules & ROLs \\
\hline \hline \multirow{4}{*}{ Pixel } & Barrel 0 & 286 & 44 \\
\cline { 2 - 4 } & Barrel 1 & 494 & 38 \\
\cline { 2 - 4 } & Barrel 2 & 676 & 26 \\
\cline { 2 - 4 } & End-Cap A & 144 & 12 \\
\cline { 2 - 4 } & End-Cap C & 144 & 12 \\
\hline \multirow{4}{*}{ SCT } & Barrels A & 1056 & 22 \\
\cline { 2 - 4 } & Barrels C & 1056 & 22 \\
\cline { 2 - 4 } & End-Cap A & 988 & 23 \\
\cline { 2 - 4 } & End-Cap C & 988 & 23 \\
\hline
\end{tabular}

Table 1: Data Formatter input readout links

will be mounted to 14 stave structures ${ }^{1}$.

\section{The Fast Tracker and Data Formatter System}

\subsection{Overview of the Fast TracKer system and FTK tower structure}

The Fast TracKer (FTK) system finds and fits tracks using the inner detector silicon layers for every event that passes the level-1 trigger decision at rates up to $100 \mathrm{kHz}$. The FTK receives the SLINK outputs from Pixel and SCT RODs, which are duplicated at the output stage of ROD modules.

In order to sustain a $100 \mathrm{kHz}$ level-1 trigger rate, FTK is organized as a set of independent engines arranged in $\eta$ - $\phi$ towers. The first step is to divide the detector into 16 sectors in $\phi$ coordinate as shown in Figure 2(a). The sectors have overlapping regions to avoid intrinsic inefficiency due to variation curvature of charged tracks at the boundary regions. The second step is to divide the $\eta$ range into four intervals: "C-Side Endcap", "C-Side Barrel", "A-Side Barrel" and "A-Side Endcap" as shown in Figure 2(b). The overlap in $\eta$ takes into account the size of finite size of the beam's luminous region in $z$.

Each FTK $\eta-\phi$ tower core processor consists of a track finder stage followed by two sequential stages of track fitters. The track finder uses pattern recognition associative memories to quickly find track candidates in coarse resolution roads. Roads which match the selection criteria are then analyzed in the track fitting stages using full resolution silicon hits. The extracted track parameters are reported to the level-2 trigger algorithm. The track finder and the first stage of track fitter make use of subset of silicon layers, while all layers are used in the final fitting to maximize fake track rejection. Using two track fitting stages results in a good balance between resource usage and tracking performance. Appendix A summarizes the details of the partitioning of the FTK tower structure.

\subsection{The Data Formatter System}

The FTK system runs the tracking algorithms using Pixel and SCT silicon hits which are sent from the RODs on SLINK fibers. Before the Pixel and SCT hits are used by the FTK core crates they must be remapped and repackaged into symmetric $\eta$ - $\phi$ towers. Data duplication is required in the overlap regions at the tower boundaries. The FTK system proposal includes a hardware-based Data Formatter system at the input of the FTK system to remap input data to match the FTK $\eta$ - $\phi$ tower structure. Considering the high level-1 trigger rate up

\footnotetext{
${ }^{1} 14$ RODs will be used to read out the module data, and 112 ROLs will be implemented where 56 links are used for ROS, and the other 56 for FTK.
} 


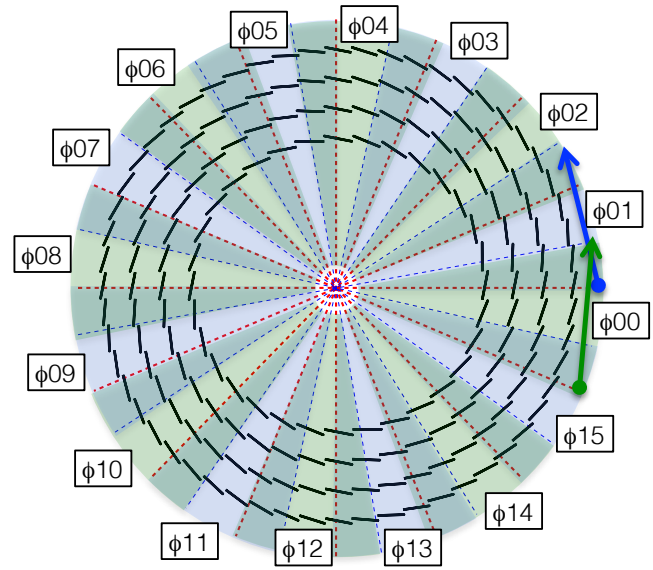

(a)

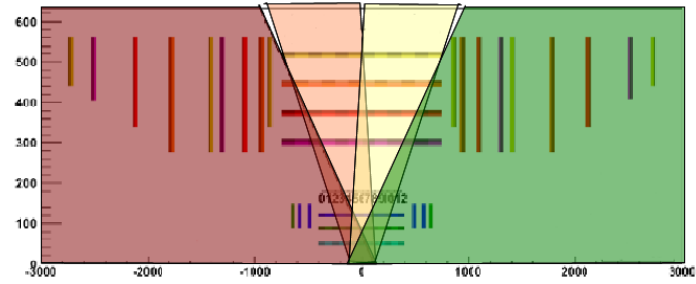

(b)

Figure 2: (a) The 16 FTK $\phi$ sectors. Note overlapping is implemented at the boundary to avoid inefficiency due to finite curvature of low momentum tracks. As example the coverage of the $\phi 00$ sector and $\phi 01$ sector are shown with green and blue arrows respectively in the figure. (b) The Four FTK $\eta$ regions. Note the significant overlap in the high occupancy central barrel regions.

to $100 \mathrm{kHz}$ and high hit occupancy of the inner detectors, the Data Formatter system is required to support massive bandwidth in the system. Recognizing that the module-ROD mapping will likely change over time, it is critical to select a backplane technology that is robust and flexible enough to handle upstream hardware configuration changes and allow for future expansion.

The Data Formatter system is required to satisfy following design requirements at the maximum level-1 trigger rate of $100 \mathrm{kHz}$ and with the maximum luminosity of $\mathcal{L}=3 \times$ $10^{34} \mathrm{~cm}^{-2} \mathrm{~s}^{-1}$ :

- Receive data from the RODs

- Remap the ATLAS inner detector geometry to match the FTK $\eta-\phi$ towers

- Send data to the 64 downstream FTK processors

- Maintain flexibility to handle upstream hardware configuration changes

In addition to the above requirements the Data Formatter system also performs clustering of Pixel and SCT hits. The conceptual diagram of the Data Formatter system is shown in Figure 3.

We have extensively analyzed and simulated data flow in the Data Formatter system. Based on the performance criteria and design requirements we conclude that a full mesh Advanced Telecom Computing Architecture (ATCA) backplane is natural fit for the Data Formatter design.

\section{The Data Formatter System}

The full mesh ATCA backplane is central to the Data Formatter system design. In the full mesh ATCA backplane all boards in the shelf are directly connected using multiple high speed serial links. Since all backplane links are point-to-point there is no need for bus arbitration, maximizing data transfer efficiency and minimizing system latency. Field programmable gate 


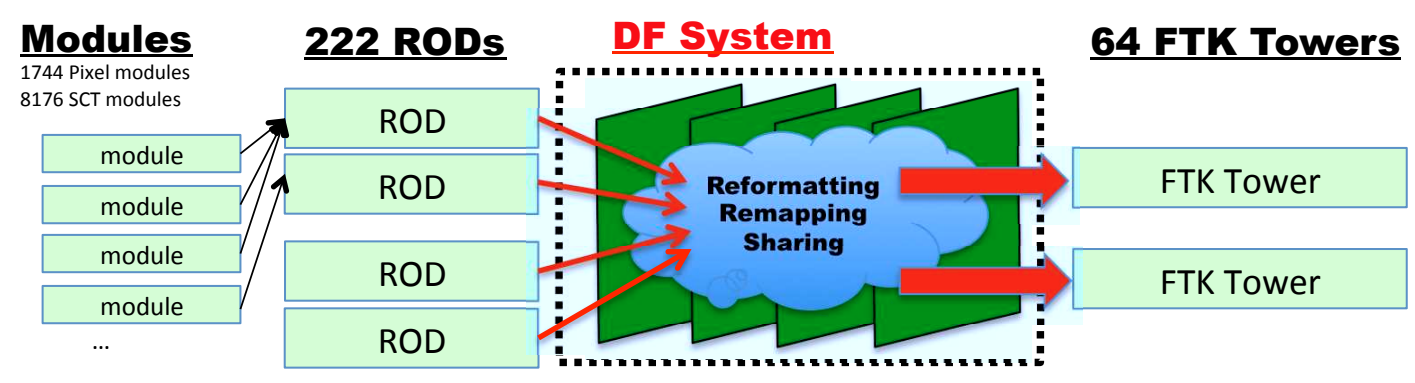

Figure 3: Data from 222 RODs to the Data Formatter and are sent to downstream 64 FTK towers after clustering, remapping, reformatting, and sharing.

array (FPGA) devices are used for the Data Formatter "processors". One FPGA is assigned for each FTK $\eta$ - $\phi$ tower, and 64 FPGAs are used in total. Each of the 64 Data Formatter FPGAs receive data from upstream RODs, share data over the ATCA backplane and intershelf links, and finally send data downstream to the FTK core crates. In following sections we introduce the conceptual design of the Data Formatter boards and how data flow influences the overall system level design.

\subsection{Conceptual Board-Level Design}

Figure 4 shows the conceptual design of the Data Formatter board. Fiber links from the Pixel and SCT RODs are received on mezzanine cards. The mezzanine cards contain FPGAs which are used to run cluster finding algorithms. Clusters from the mezzanine card are fed into two large FPGAs on the Data Formatter board (yellow lines). These two FPGAs share data over an on-board local bus (orange line), the full mesh backplane (pink line), and bidirectional optical fiber links driven by the rear transition module (RTM) (green line). Fiber optic transceivers on the RTM are used to send data downstream to the FTK core processor crates as well (blue line).

\subsection{Data Sharing Paths}

The Data Formatter system is equipped with three types of internal communication paths to connect the 64 FPGAs in the system. The first path is a local bus which connects the FPGAs on the same Data Formatter board (Figure 5(a)). The second path is the full mesh fabric interface in the ATCA backplane. All FPGAs in the shelf are directly connected over high speed serial links in the full mesh backplane (see Figure 5(b)). Data Formatter boards are organized in order to minimize data sharing across the backplane. There are, however, cases where FPGAs must share data across shelf boundaries. Inter-shelf communication occurs using fiber optic transceivers on the RTM (Figure 5(c)). Using the three types of communication links each FPGA has a path to all other FPGAs in the system.

\subsection{System-Level Design of Data Formatter System}

The Data Formatter system will consist of four 14-slot ATCA shelves, 32 main boards, 64 FPGAs, 32 RTMs, and up to 128 mezzanine cards. Data Formatter FPGAs use integrated multi-gigabit serial transceivers to interface to the ATCA backplane and fiber transceivers on the RTM. The FPGA serial transceivers are rated for speeds up to $10 \mathrm{~Gb} / \mathrm{s}$. Figure 6 illustrates the substantial connectivity achievable with the Data Formatter system. Each line 


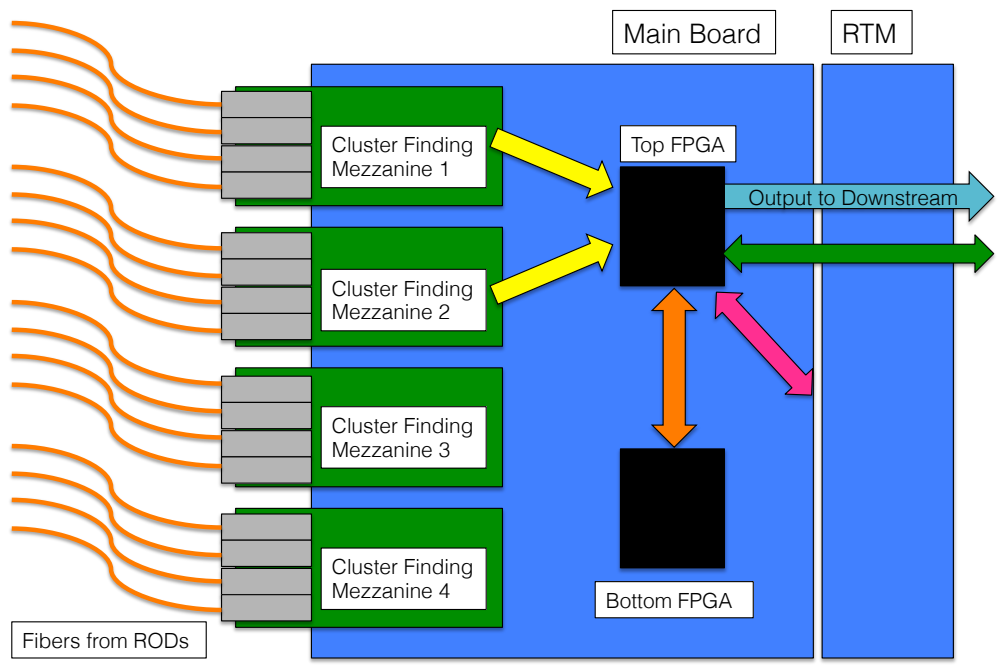

Figure 4: Data Formatter conceptual board design. Each Data Formatter board supports up to four cluster finder mezzanine cards and two FPGAs. The RTM board contains fiber optic transceivers for sending data downstream to the FTK core processor crates.

in the diagram represents a serial link up to $10 \mathrm{~Gb} / \mathrm{s}$. Inter-shelf links shown in this figure accurately reflect the results of our bandwidth study (refer to Section 8.1.2).

\subsection{Input and Output Flexibility}

The Data Formatter system will support up to 128 mezzanine cards. Each mezzanine card will support up to four SFP+ optical transceivers for a total of 512 input links. This is significantly more than the current 222 optical fibers from Pixel and SCT RODs which leaves room for future expansion. If more than 512 input links are required then additional Data Formatter boards and mezzanine boards may be installed. RTM boards support up to eight QSFP + and six SFP+ optical transceivers; half of the transceivers are directly connected to the Top FPGA and the other half are directly connected to the Bottom FPGA. Optical or copper serial transceiver modules may be installed in any SFP+ or QSFP+ location on the RTM. 


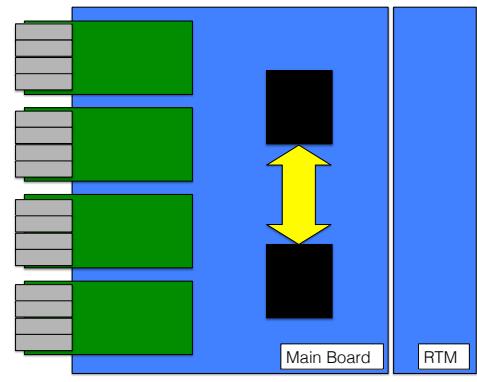

(a)

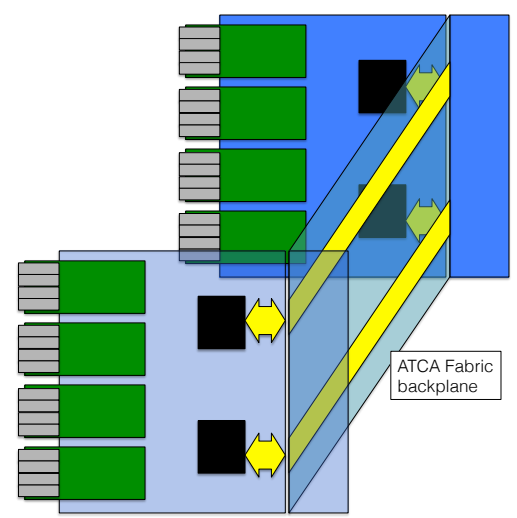

(b)

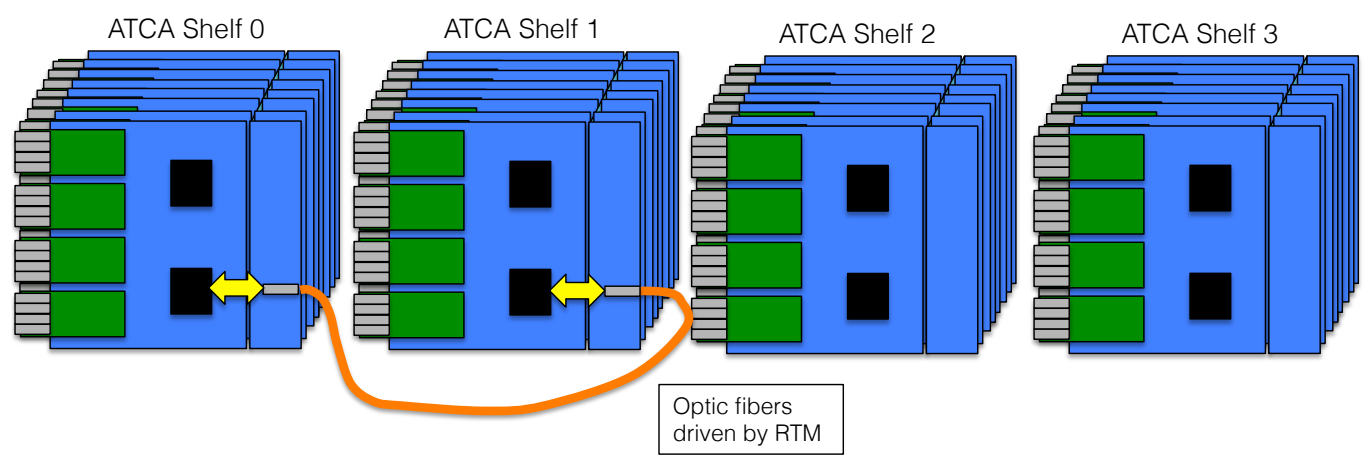

(c)

Figure 5: Three types of communication in the Data Formatter. (a) A local bus connects the two FPGAs on the board. (b) All FPGAs in the shelf are directly connected using the ATCA backplane fabric interface. (c) Inter-Shelf communication uses RTM transceivers.

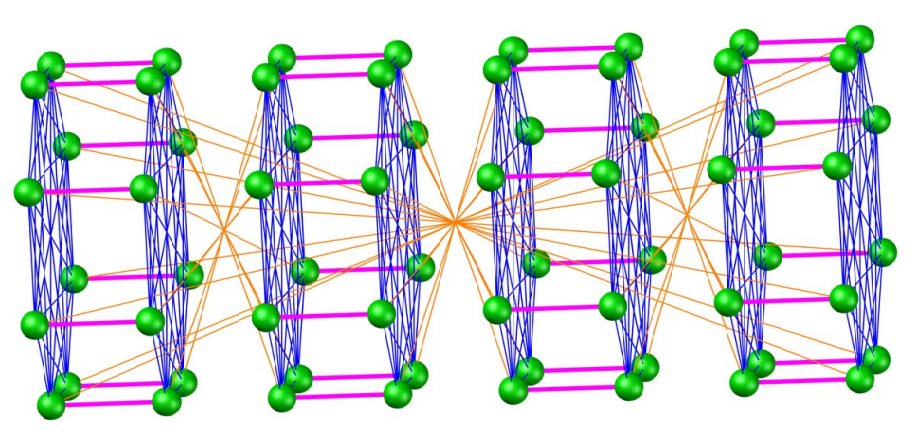

Figure 6: A 3D representation of FPGA interconnects in the Data Formatter system. 64 FPGAs (green) are connected through the ATCA backplane Fabric Interface (blue), local buses (purple) and inter-shelf links (orange). Each FPGA uses one inter-shelf link. This diagram assumes 4 shelves with 8 Data Formatter boards per shelf. For this picture, the initial fiber connection introduced in Section 8.1.2 is shown. An animated version is available [13]. 


\section{Data Formatter Board}

\subsection{Design Overview}

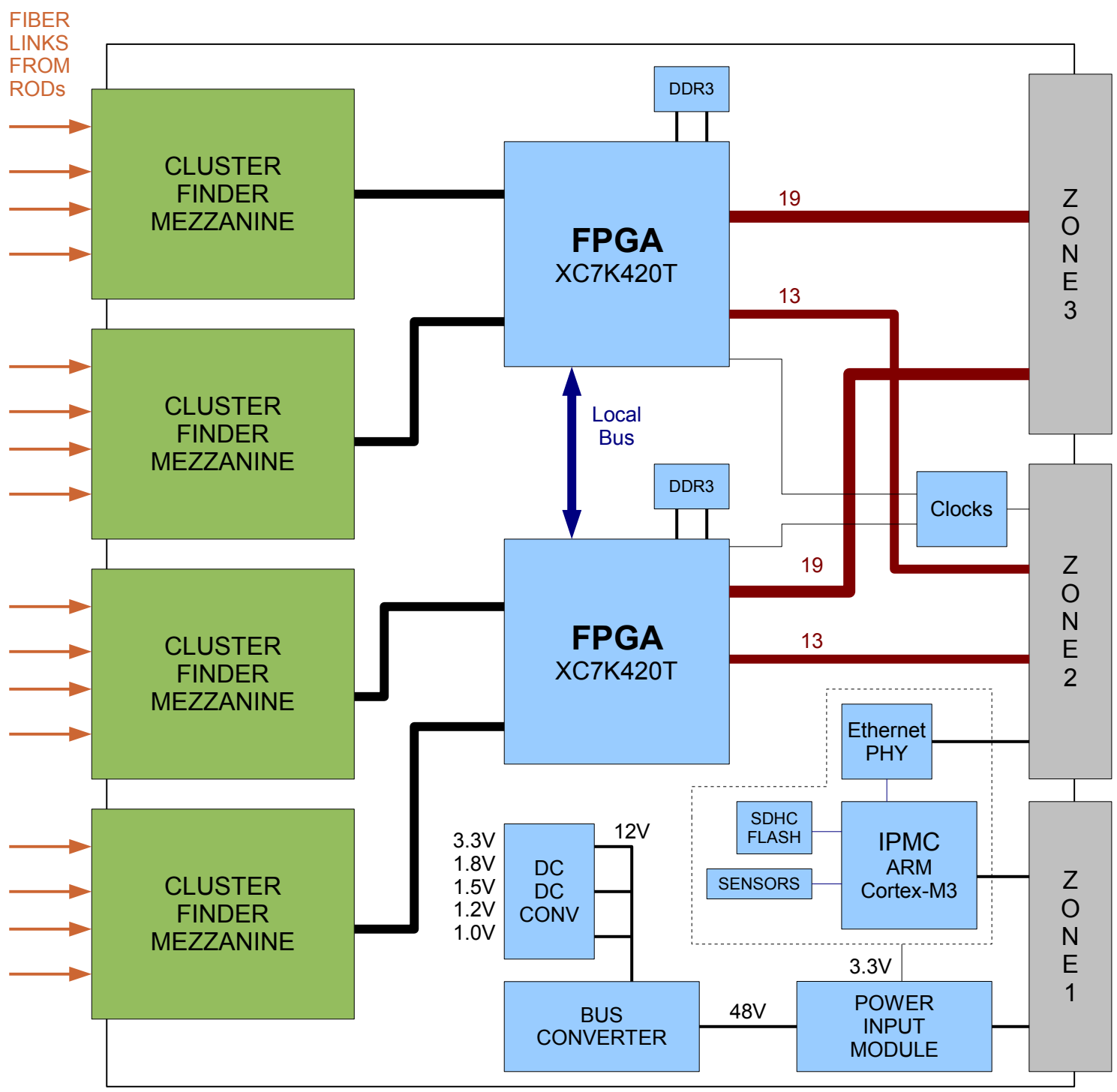

Figure 7: The Data Formatter board block diagram.

The section introduces functions and design details of the Data Formatter board. The Data Formatter block diagram is shown in Figure 7. As described in Section 4, two FPGAs (Section 5.2) are mounted on each Data Formatter board so as to cover two FTK $\eta-\phi$ towers. FPGAs are the Data Formatter's processing engine: they receive cluster data from the mezzanine cards, share data with other FPGAs (i.e. other FTK $\eta-\phi$ towers), and send the data downstream to the FTK core processor crates. Additional circuitry on the Data Formatter board is used to power and configure the FPGAs, implement slow controls and monitoring, provide clocks and synchronization, and support the ATCA system management protocol. 


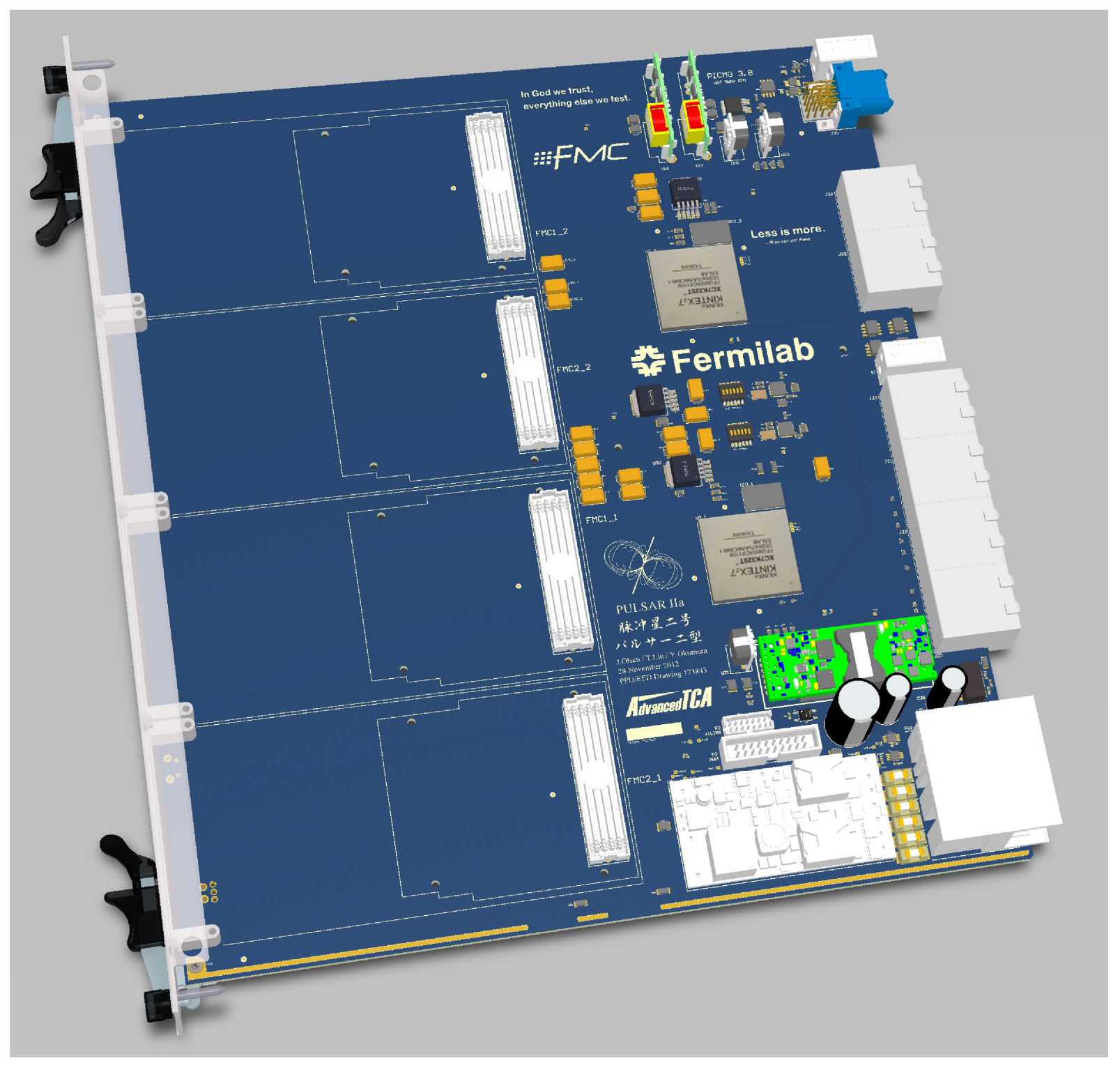

Figure 8: The Data Formatter Board 3D model in Altium layout software.

\subsection{Core Processing Engines (FPGAs)}

Two large FPGAs form the processing core of the Data Formatter board. These two FPGAs are symmetric and have identical pinouts. The Xilinx Kintex XC7K420T FPGA features 32 high-speed serial (GTX) transceivers which support data rates up to $12.5 \mathrm{~Gb} / \mathrm{s}^{2}, 420 \mathrm{k}$ logic cells, 400 IO pins, and 4Mbytes of internal dual port RAM and FIFOs. Firmware studies for the FPGAs are mentioned in Appendix E.

\subsubsection{Mezzanine Card Interface}

Fiber optic links from the Pixel and SCT RODs are received on the Mezzanine Cards. Each FPGA connects to two Mezzanine Cards as shown in Figure 7. The Mezzanine Card interface uses the FMC (VITA 57) standard and is based on low voltage differential pair (LVDS) signals. Refer to Section 6 for details on this interface.

\footnotetext{
${ }^{2}$ The speed depends on choice of FPGAs varying from $6.6 \mathrm{~Gb} / \mathrm{s}$ to $12.5 \mathrm{~Gb} / \mathrm{s}$
} 


\subsubsection{Fabric Interface}

The ATCA backplane full mesh Fabric Interface supports communication between two FPGAs (i.e. FTK $\eta-\phi$ towers) assigned to the different boards in the same shelf. These connections are shown with dark red lines linking the FPGAs to Zone-2 connector in Figure 7. FPGAs use their internal high speed serial transceivers (GTX) to communicate over the Fabric Interface. In a full mesh ATCA backplane all slots are directly connected with dedicated channels. Each channel consists of four full-duplex ports. Each backplane port is rated for up to 10Gbps.

Data Formatter boards use two of the four available ports on the ATCA full mesh backplane. The Top FPGA connects to port 0 of fabric channels 1 through 13 (see Appendix B and [5]). The bottom FPGA connects to port 1 of fabric channels 1 through 13 . This means that Data Formatter boards may be installed in slots 3-14. All top FPGAs are directly connected and all bottom FPGAs are directly connected through the backplane Fabric Interface.

An efficient, lightweight yet robust communication protocol will be used on the serial links connecting FPGAs. We are currently evaluating the Aurora protocol developed by Xilinx.

\subsubsection{Inter-FPGA link on board (Local Bus)}

The local bus on the board supports communication between two FPGAs (i.e. FTK $\eta-\phi$ towers) assigned to the same boards. A pair of local buses connects the two FPGAs on each Data Formatter board. The local bus is shown with blue lines linking the two FPGAs in Figure 7. The bus consists of LVDS differential pairs, and the maximum data transfer rate of each line varies from $1.2 \mathrm{Gbps}$ to $1.6 \mathrm{Gbps}$, depending on the FPGA choice. The number of pairs of the differential LVDS is still flexible and to be determined so that the bandwidth satisfies the given requirement for high luminosity runs.

\subsubsection{RTM Interface}

The RTMs support data transmission downstream to the FTK processors and data sharing across shelf boundaries. The connection to RTM is shown with red lines linking the FPGAs to Zone-3 connector in Figure 7. Each FPGA has 19 GTX transceivers reserved for RTM connections. 17 GTX channels are reserved for data transmission downstream to FTK, and 2 GTX channels are reserved for inter-shelf communication. RTM optical and copper pluggable transceivers support bidirectional data rates up to $10 \mathrm{~Gb} / \mathrm{s}$ for a maximum bandwidth of $380 \mathrm{~Gb} / \mathrm{s}$ per RTM. Refer to Section 7 for the RTM design details.

\subsubsection{DDR3 Memory}

Each FPGA has 835 36k-bit internal BlockRAMs which may be configured as FIFOs ROMs, single-port RAM, or dual-port RAM. In the event more memory is required for event buffering an external DDR3 memory device is included in the design. This DDR3 memory chip is $256 \mathrm{MBytes}$ and has a 16 bit data bus operating at $800 \mathrm{Mb} / \mathrm{s}$ for a maximum data rate varying from $6.6 \mathrm{~Gb} / \mathrm{s}$ to $12.5 \mathrm{~Gb} / \mathrm{s}$, depending on the FPGA speed grade. The effective data rate will vary due to DDR3 signaling overhead but is expected to be nearly $70 \%$ of maximum for block sequential transfers and $40 \%$ for random access. The Xilinx Memory Interface Generator (MIG) tool creates a firmware interface for the DDR3 memory chip.

\subsection{Microcontroller}

A small microcontroller is used for various board management functions such as slow controls, board monitoring, FPGA configuration, and ATCA shelf management. The microcontroller 


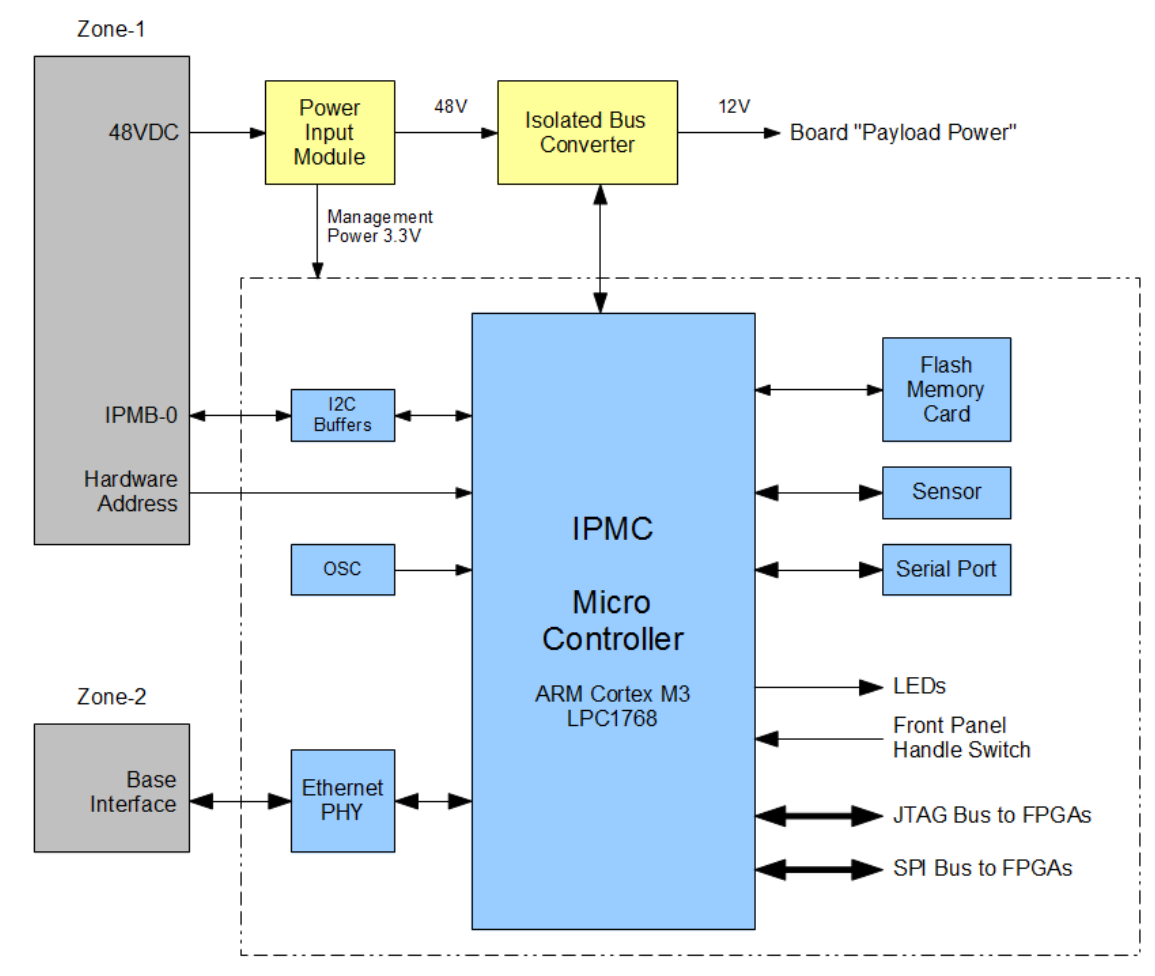

Figure 9: The Data Formatter microcontroller and associated circuitry.

communicates with the ATCA shelf manager boards through the Zone-1 connector. The Zone2 connector Base Interface enables the microcontroller to interface with Ethernet networks via the hub board located in slot 1. Microcontroller connections are shown in Figure 9.

An ARM Cortex-M3 microcontroller has been selected for use on the Data Formatter board. This microcontroller runs at up to $100 \mathrm{MHz}$ and features $512 \mathrm{kB}$ Flash program memory, $64 \mathrm{kB}$ RAM, an Ethernet MAC interface, and various on-board peripherals such as I2C, RS232 UARTs, and SPI.

The microcontroller implements the ATCA Intelligent Platform Management Interface (IPMI) and the slow controls interface (See Appendix B.5 for more details).

\subsubsection{IPMI Controller}

ATCA hardware achieves high availability through a robust hardware management scheme. Redundant shelf manager boards communicate with boards and components over redundant I2C buses. The IPMI controller (IPMC, see Appendix B) is used to report board telemetry data to the shelf manager and coordinates the hot swap power sequencing.

The microcontroller has three I2C buses. The first two I2C buses are used for the IPMI buses (IPMB). The third I2C bus connects to a temperature sensor, the main power bus converter, and the RTM.

A commercial IPMC software reference design is available, however this code is closedsource and has expensive licensing terms. A group of Engineers from various HEP laboratories have formed an ad-hoc committee and we are working towards a simple, modular and deviceindependent open-source solution.

The IPMI protocol includes support for firmware updates. However, the "remote update" functionality should not be used for downloading FPGA firmware because the I2C data rates are very slow, on the order of $100 \mathrm{kbps}$. 


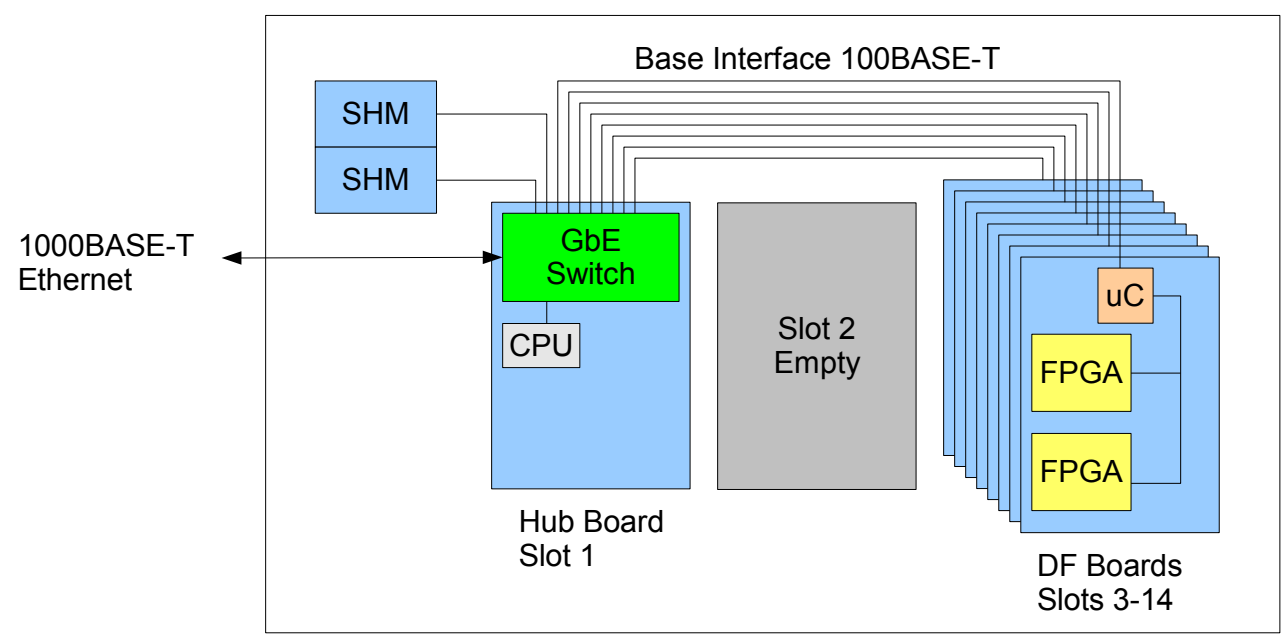

Figure 10: A typical shelf configuration. The Slot 1 Base Interface hub connects the Shelf Manager boards and the Data Formatter board microcontrollers and provides one or more external Gigabit Ethernet ports. Note that the high speed Fabric Interface is not shown here. An optional CPU on the hub may be used as a "crate controller" if required.

\subsubsection{Ethernet Interface}

The microcontroller features a hardware-based 100BASE-T Ethernet MAC which enables the use of TCP-IP protocols such as WWW, Telnet and FTP. This interface is intended for downloading FPGA firmware and slow controls. Initial testing with our Cortex-M3 development board has demonstrated FTP transfer rates of up to $2 \mathrm{MB} / \mathrm{s}$.

A 100BASE-T Ethernet PHY chip (National DP83848) connects the microcontroller to the Base Interface channel 1 on the Zone-2 connector. Note that Base Interface channel 2 is not used. This means that an ATCA hub board (or "switch blade") may be installed only in slot 1 as shown in Figure 10.

\subsubsection{Flash Memory}

A removable micro-SDHC flash memory card is used for local file storage. FPGA firmware image files will be downloaded over the Base Interface Ethernet interface and stored on the flash card. Alternatively the flash card may be removed from the Data Formatter and inserted into a laptop the files transferred directly to the card. The flash card uses the FAT32 file system and supports long file names.

\subsubsection{FPGA Interface}

The microcontroller drives the JTAG bus which connects to the two main FPGAs. The JTAG bus is used for programming the FPGAs. After the FPGAs are programmed the microcontroller uses an SPI bus to access internal registers and buffers. The maximum frequency of the SPI bus clock and JTAG clock is $50 \mathrm{MHz}$. It should be noted that this interface is intended for SLOW controls and monitoring. It is not intended for high speed data transfers. 


\subsubsection{Board Sensors and RTM Management}

The microcontroller's third I2C bus connects to a sensor chip (LTC2990) which contains monitors ambient air temperature and the $+3.3 \mathrm{~V}$ management power supply. This $\mathrm{I} 2 \mathrm{C}$ bus also connects to the 12VDC bus converter, which monitors input and output voltages, currents, and temperature. Finally the I2C bus connects to the RTM management connector J30.

\subsubsection{USB Serial Port}

The microcontroller serial port is used for debugging and configuring the network parameters. A small USB to RS232 interface chip (Silabs CP2102) is used and a micro-USB connector is provided on the Data Formatter front panel. The serial port is configured as $115.2 \mathrm{k}$ baud, 8 data bits, 1 stop bit, no parity.

\subsubsection{Microcontroller Software}

The microcontroller will need to switch efficiently between many different tasks such as: IPMI protocol handling, TCP-IP networking, slow monitor management, flash card file systems and general bit-banging on the I/O pins. The ARM community has a wide selection of smallfootprint, efficient, real-time operating systems (RTOS) that are ideal for our application. We have purchased the KEIL MDK-Professional tools and are developing our software around KEIL RTX RTOS kernel.

\subsection{Clock Distribution}

Data Formatter boards are data driven and essentially do not require synchronizing to a master clock. However, we have built in provisions to synchronize Data Formatter boards across the ATCA backplane.

\subsubsection{Local Oscillators}

The Kintex FPGAs require extremely clean clocks to drive the high speed serial transceivers (GTX). Two clocks are generated locally using high quality precision oscillators and programmable low-jitter PLL-based clock buffers.

The main clock is $200 \mathrm{MHz}$ and is intended for general purpose clocking in the FPGAs. This clock also drives the DDR3 controller in the FPGA. An additional clock generator drives two reference clocks to the FPGA GTX refclk input pins.

\subsubsection{Backplane Clocks}

Shelf level synchronization may be achieved using the ATCA backplane Synchronization Interface, which consists of 6 differential clock signals bused to all slots. The first 4 clocks (CLK1A, CLK1B, CLK2A, and CLK2B) are reserved for standard telecom frequencies. Backplane clocks CLK3A and CLK3B are user-defined and are connected to the Data Formatter FPGAs through multi-point LVDS transceivers. (A Data Formatter FPGA may act has the clock master and drive these clock lines.)

If shelf-wide synchronization with the trigger DAQ is required there are a few options. One option is to develop a mezzanine card with a CERN TTC receiver on it. This special mezzanine card could be plugged into an available Data Formatter board and the firmware could pass the DAQ timing signals through the FPGA to the backplane bus. Another option would be to develop a simple TTC receiver ATCA board and install this special board in slot 2 . 


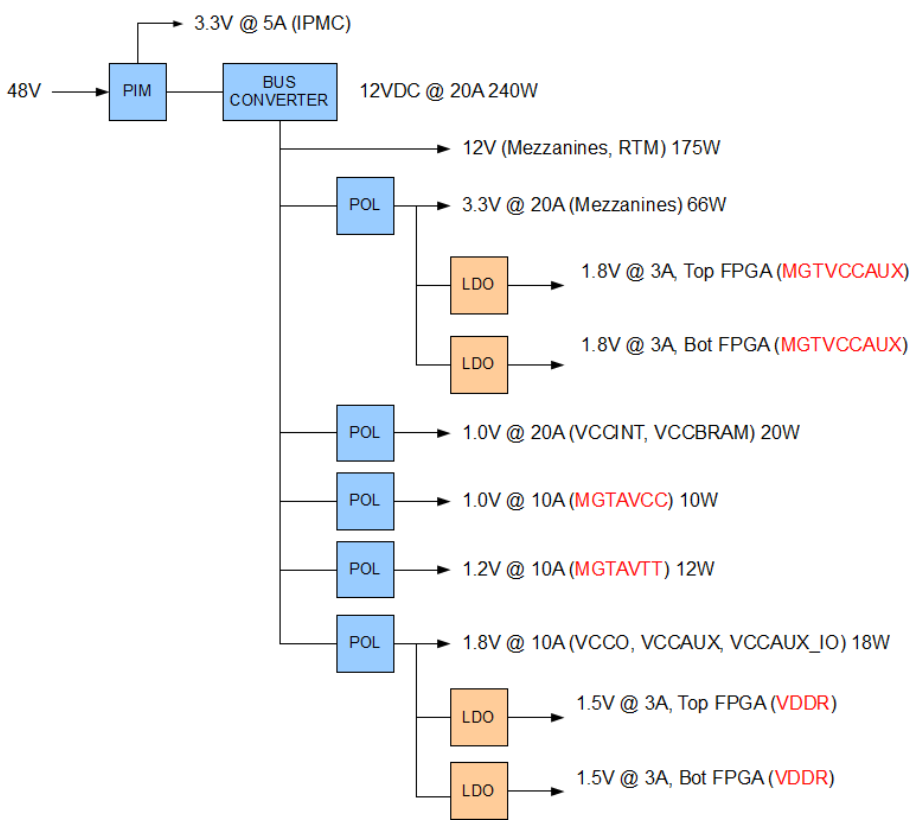

Figure 11: The Data Formatter power distribution. POL refers to a "Point-of-Load" switching regulator. LDO refers to a "Low Drop Out" linear regulator.

\subsection{Power Distribution}

\subsubsection{Power Distribution Hierarchy}

The ATCA backplane supplies dual redundant -48VDC power feeds. These buses are fused, filtered, and converted to the extremely quiet and well-regulated 1.0VDC required by the Kintex FPGAs. The power distribution system is shown in Figure 11.

Immediately after the Zone-1 power connector the power feeds are fused and connected to an ATCA-specific Power Input Module (PIM, GE PIM300FZ). The PIM module filters the power feeds and combines them using diodes. The PIM also generates $+3.3 \mathrm{~V}$ for powering the IPMC microcontroller and associated circuitry.

An isolated bus converter (GE EBDW020A0B) steps the 48VDC down to 12VDC rated for up to 20A. This 12 VDC supply powers the RTM and Mezzanine cards. Smaller Point-of-Load switching regulators (GE NQR010A and NSR020A0) generate lower voltages. A few power rails are generated with linear regulators (Linear Tech LT1764A). FPGA GTX transceivers require very quiet power supplies and may not be shared with the general purpose digital power rails.

\subsubsection{Power Control Sequence}

Immediately upon board insertion the PIM provides power to the IPMC microcontroller. The IPMC and shelf manager negotiate power requirements and once that process completes the shelf manager allows the IPMC to enable the bus converter, which powers up the board and RTM. (Xilinx Kintex FPGAs do not require any specific power supply sequencing as long as each power rail ramps up is monotonic.)

To remove a board from the ATCA shelf the user must first open the lower handle just slightly so that a microswitch is opened. The IPMC sends a message to the shelf manager and slowly blinks the blue "HS" LED. Once the shelf manager has responded the IPMC disables the bus converter and the board may be moved from the shelf. 


\subsection{Prototype Board}

A prototype Data Formatter board is currently being fabricated. The prototype board design is very close to the production board but differs in the FPGA choice. In consequence it differs in GTX transceiver connectivity to the RTM and Fabric Interface, too. The block diagram is shown in Figure 12.

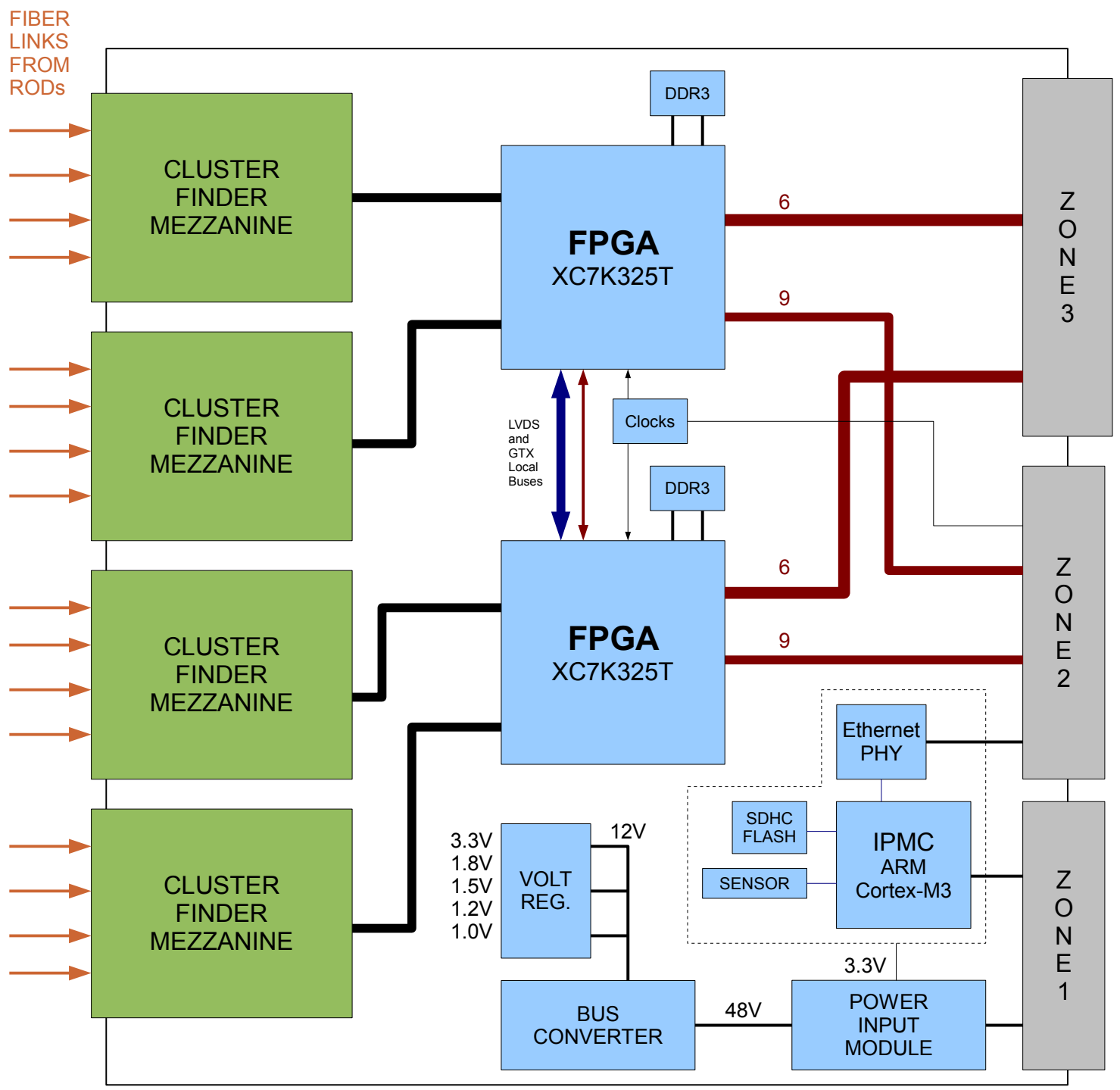

Figure 12: The prototype Data Formatter board uses two smaller FPGAs with reduced RTM and Fabric Interface connectivity.

The prototype Data Formatter uses the Xilinx Kintex XC7K325T device in the FF900 package while the production board will use the Kintex K420T in a FF1156 package. All Kintex FPGAs are currently in full production. The Kintex K325T FPGAs have 16 GTX transceivers. Six GTX transceivers are used for the RTM, 9 GTX transceivers are used for the Fabric Interface, and the last GTX transceiver is used for the FPGA local bus. The limited Fabric Interface connections means that up to eight prototype boards may only be installed in slots 3-10. Each FPGA connects to one QSFP+ transceiver (T1/B1) and two $\mathrm{SFP}+$ transceivers (T5/B5 and T6/B6) on the RTM. 


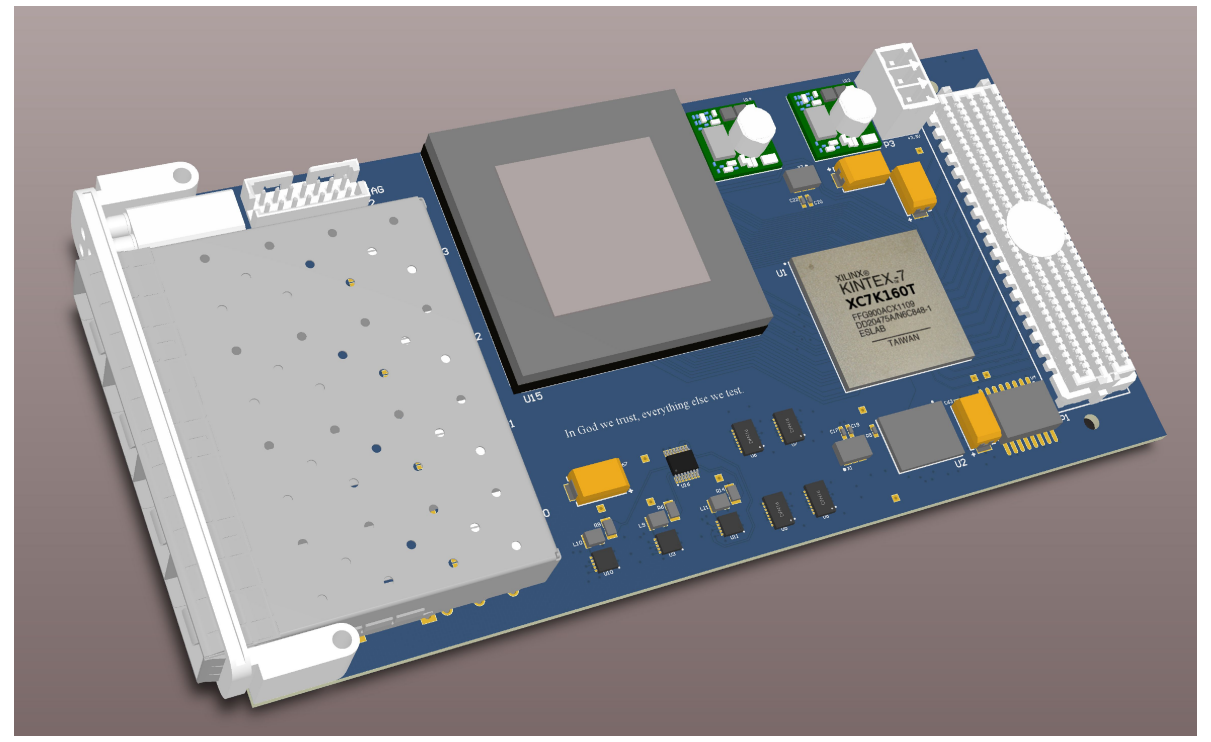

Figure 13: A test Mezzanine Card. This design features four SFP+ pluggable serial transceivers, a small Kintex FPGA, configuration flash memory, DDR3 memory, power supplies, local oscillators, a test socket and FMC connector.

\section{Mezzanine Cards}

\subsection{Introduction}

This section focuses on the interface between Mezzanine Cards and Data Formatter boards. The connector mechanical design, bandwidth, power, pin assignments, configuration, and clocking will be discussed. Specific information pertaining to the FTK cluster finding Mezzanine Card is covered in Section 6.8.

The Data Formatter board supports up to four Mezzanine Cards as shown in Figure 7. Data Formatter Mezzanine Cards use the FPGA Mezzanine Card (FMC) connector which is defined by the VITA 57.1 specification [9]. Figure 13 shows a test Mezzanine Card design.

The FMC connector is used on many FPGA development boards from Xilinx, Digilent, and others [10]. By using an FMC connector it is possible to use commercially available FPGA development boards to test prototype Mezzanine Cards. Likewise, the Data Formatter boards are compatible with a growing selection of FMC I/O modules [11]. 


\subsection{FMC Connector}

The FMC Connector has 400 pins and provides connectivity for up to:

- 160 single-ended or 80 differential user defined signals

- 10 GTX transceivers

- 2 GTX clocks

- 2 clocks

- 4 differential clocks

- 159 ground and 15 power connections

The connections between the FMC connector and the Data Formatter board FPGAs implements a subset of this connectivity:

- 58 differential user defined pairs:

- 34 LA pairs (LA00-LA33)

- 24 HA pairs (HA00-HA23)

- 2 differential clocks

- 159 ground and 10 power connections

The FMC connector is available in High and Low Pin Count versions. The High Pin Count (HPC) has all 400 pins loaded, while the LPC version fills only rows C, D, G, and H for a total of 160 pins. The Data Formatter supports a subset of the HPC signals. The Data Formatter FMC connector pinout is listed in Appendix C.1. The Data Formatter system does not use the connectivity for GTX.

\subsection{Signal Levels and Bandwidth}

All mezzanine card connector signals are routed directly to the Data Formatter FPGAs. The Data Formatter FPGA I/O banks have a VCCO voltage of $+2.5 \mathrm{~V}$. Signal levels must not exceed $+2.5 \mathrm{~V}$ on any signal pin or else the Data Formatter FPGAs may be damaged.

Single ended signals should use the LVCMOS25 I/O standard. Differential signals should use the LVDS or LVDS25_25 I/O standards. If required a differential pair may be treated as two independent single-ended signals, however these traces are routed as differential striplines and crosstalk may be an issue.

Modern FPGAs support data rates exceeding 1Gbps per LVDS pair. If all LA and HA banks are used the total bandwidth on the HPC connector is over 70Gbps using Xilinx Kintex FPGAs in the slowest (-1) speed grade.

\subsection{Mezzanine Card Dimensions}

The Mezzanine Card follows the standard CMC/PMC dimensions of $74 \mathrm{~mm} \times 149 \mathrm{~mm}$. The mechanical drawing is shown in Appendix C.3.

The Mezzanine Card will use a standard CMC/PMC style bezel which is $74 \mathrm{~mm}$ wide. The inter-board spacing (or connector "stack height") is $10 \mathrm{~mm}$. An EMC gasket may be used. With a standard CMC/PMC style bezel there is just enough room to fit four SFP optical transceivers and some LEDs. 
The component side of the Mezzanine Card faces the Data Formatter board when installed. The Data Formatter boards have no components in this area therefore the maximum component height is $10 \mathrm{~mm}$. It is possible to place parts on the "solder side" of the mezzanine card, as long as the total height does not exceed the ATCA maximum component height of $22.5 \mathrm{~mm}$.

\subsection{Power}

The Data Formatter board connects the VADJ pins to 3.3V. According to the VITA specification the FMC connector is rated for up to $4 \mathrm{~A}$ on the VADJ pins, up to $1 \mathrm{~A}$ on the 12VDC pins, and up to $3 \mathrm{~A}$ on the $3 \mathrm{P} 3 \mathrm{~V}$ pins. Therefore the maximum power available to a mezzanine card is $35 \mathrm{~W}$. Mezzanine Cards do not support hot swap. The standby power pin 3P3AUX is not connected on the Data Formatter board.

\subsection{Management and JTAG Interfaces}

When the Mezzanine Card is installed it should ground the PRSNT_M2C_L pin low. Slow controls may use the I2C bus signals SCK and SDA. The SCK and SDA signals should be pulled up to $+2.5 \mathrm{~V}$ through $4.7 \mathrm{k}$ resistors on the mezzanine card.

Mezzanine Cards may use the JTAG interface for programming FPGAs. The Data Formatter is the JTAG bus master and may be used for programming devices on the Mezzanine Cards. However, as this JTAG interface is very slow it is highly recommended that Mezzanine Cards store firmware images locally in flash memories or PROMs. Mezzanine Card designers are strongly encouraged to provide a separate JTAG programming header for local programming and debugging. JTAG signals should use the LVCMOS_25 I/O standard.

\subsection{Clocks}

The FMC connector has two dedicated differential output clocks, CLK0_M2C and CLK1_M2C. These signals are routed to global multi-region clock-capable (MRCC) pins on the Data Formatter FPGAs.

The Data Formatter board can drive a differential clock to the mezzanine board on any signal pair in the LA or HA banks. It is recommended to route the LA00, LA01, HA00, HA01 signals to clock-capable pins on the mezzanine card FPGAs.

It is highly recommended that Mezzanine Card designs include a high quality low-jitter local oscillator to provide a clean reference clock source if multi-gigabit serial links are used.

\subsection{Cluster Finder Mezzanine Card}

The FTK_IM Mezzanine Card receives up to four SLINK fibers and unpacks the ROD data and performs SCT and Pixel detector clustering. The prototype mezzanine card contains four SFP optical transceivers, two FPGAs, and DDR memories and has been developed by INFN $[4]$.

The prototype FTK_IM mezzanine card has four 24-bit parallel data buses (A,B,C, and D) and associated control signals (HOLD, DV). These signals are single-ended and single-data rate synchronous to a $40 \mathrm{MHz}$ output clock. Additional pins are used for status, control and other user defined signals.

Using the FMC connector and high speed LVDS signaling it is possible to expand the four output buses to 32 bits wide and still have many signals left for other purposes. Modern FPGAs include serializer-deserializer logic built into the I/O pin. For example, the Xilinx 
ISERDES2 and OSERDES2 primitives are capable of serializing and deserializing up to 8 data bits per LVDS signal pair at rates exceeding 1Gbps [14].

\subsection{Prototype mezzanine card}

A test Mezzanine Card has been designed to test the Data Formatter prototype system (Section 5.6). Figure 13 shows the picture of the test mezzanine card to be used for prototype board tests. 


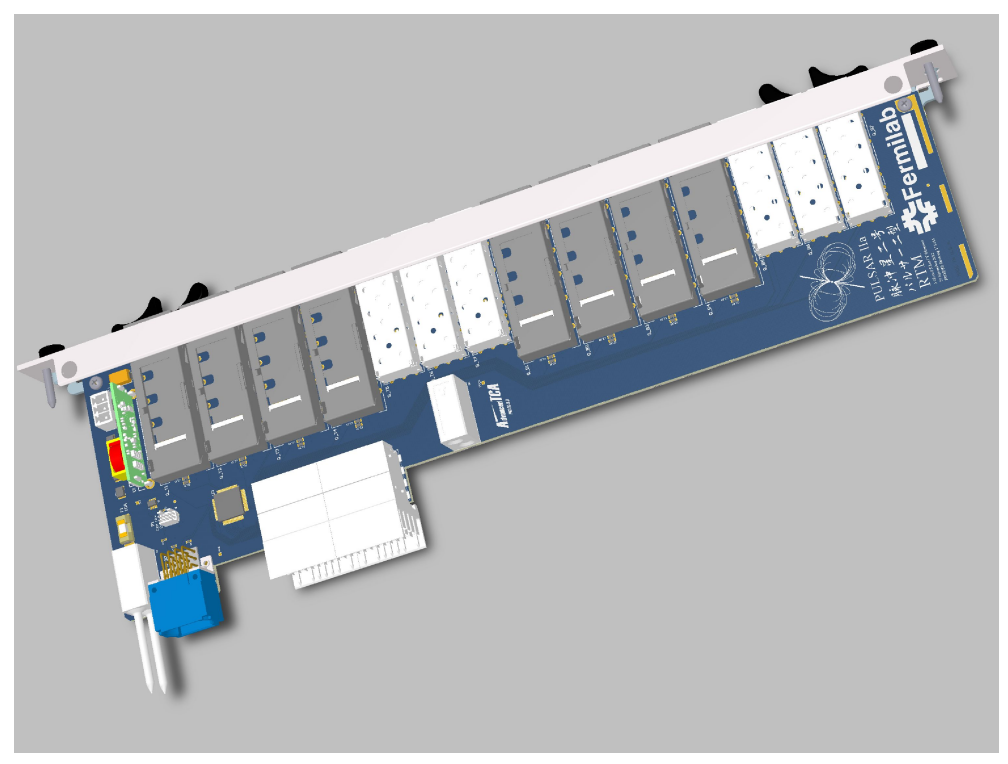

Figure 14: Data Formatter Rear Transition Module (RTM). Up to 8 QSFP and 6 SFP transceivers are supported.

\section{Rear Transition Module}

We make use of the Rear Transition Module (RTM) to operate the bi-directional high speed serial link drivers of SFP + and QSFP+ modules. In the Data Formatter system it is used to transmit data downstream to the FTK core crates. Serial transceivers on the RTM are also used for communication between ATCA shelves. Eight QSFP+ and six SFP+ transceivers are used on each RTM. The RTM design is shown in Figure 14. This board complies with the new PIGMG 3.8 Zone-3A specification [6] which defines the connectors, board mechanics, power and management.

\subsection{Transceiver Modules}

Pluggable transceivers on the RTM are used for inter-shelf data sharing and for downstream transmission to the FTK Aux Cards (AUX) and Second Stage Boards (SSB). Each Data Formatter FPGA drives 19 GTX 10Gb/s transceivers to the RTM. Up to four Quad Small Form Factor Pluggable (QSFP+) transceivers and up to 3 Small Form Factor $(\mathrm{SFP}+)$ per FPGA are supported.

QSFP transceivers have four independent full-duplex channels in a $18 \mathrm{~mm} \times 52 \mathrm{~mm} \times 8.5 \mathrm{~mm}$ package. Channel bandwidth is nominally $3.125 \mathrm{~Gb} / \mathrm{s}(10 \mathrm{~Gb} / \mathrm{s}$ in QSFP + ). Single-mode fiber, multi-mode fiber, and lower-cost copper cable QSFP transceivers are available. SFP transceivers have a single full-duplex channel in a $13 \mathrm{~mm} \times 56 \mathrm{~mm} \times 8.5 \mathrm{~mm}$ package. Channel bandwidth is nominally 1.25 to $4.25 \mathrm{~Gb} / \mathrm{s}(10 \mathrm{~Gb} / \mathrm{s}$ in SFP+). Single-mode and multi-mode fiber versions are available. All transceivers have a $\mathrm{I} 2 \mathrm{C}$ interface which allows the host to read manufacturer ROM data and operating parameters such as temperature, voltage, and optical signal strength.

\subsection{Mechanical Dimensions}

The RTM board measures $322.25 \mathrm{~mm} \times 92 \mathrm{~mm}$ as per the PICMG 3.8 specification. A rear panel and ejector handle will also be used. No backplane is used in the Zone-3 region; the RTM connectors mate directly with connectors on the Data Formatter board. 


\subsection{RTM Power}

Both $+12 \mathrm{~V}$ and $+3.3 \mathrm{~V}$ power is supplied through the management connector (P30 on the Data Formatter and RP30 on the RTM). The $+3.3 \mathrm{~V}$ power is limited to just a few watts and is intended only to power the RTM management circuitry. Both the $+3.3 \mathrm{~V}$ management power and the $+12 \mathrm{~V}$ main power are controlled by the Data Formatter IPMC microcontroller in accordance with the PICMG 3.8 specification. The RTM will support hot swap and will use a microswitch to monitor the position of the lower handle.

A non-isolated DC-DC converter is used to step down the $+12 \mathrm{~V}$ main power to $+3.3 \mathrm{~V}$ on the RTM. The $+3.3 \mathrm{~V}$ rail will be used for powering the transceivers. When all transceivers are installed and operating we estimate total RTM power consumption to be on the order of $20 \mathrm{~W}$.

\subsection{Management Interface}

The Data Formatter microcontroller's third I2C bus connects to the P30/RP30 connector. We intend to implement a local IPMI bus (IPMB-L) protocol on this bus and use it to monitor temperature, power and link status for all transceivers located on the RTM. A small ARM Cortex-M3 microcontroller (LPC1317) is used to handle the IPMI protocol. According to the PICMG 3.8 specification the RTM shall appear as an MMC controller to the front board IPMC microcontroller.

\subsection{Channel Assignments}

The PICMG 3.8 specification defines three ADF data connectors for the Zone-3A RTM interface. The Data Formatter RTM uses two of these connectors (J32 and J33). Each ADF connector has 40 differential pairs, which are divided into 20 channels numbered 0 through 19. The top FPGA connects to J32 and the bottom FPGA connects to J33. Refer to Appendix D.1 for details.

\subsection{Prototype Board}

The first batch of ten RTM boards have been produced. We are currently writing software for the RTM microcontroller. 


\section{Bandwidth Requirements}

The study to evaluate bandwidth requirements in the Data Formatter System will be presented in this section. A software model of the Data Formatter has been created so that data flow in the system may be simulated using actual event data records taken in 2012. The initial hardware configuration used in this study is explained in Section 8.1, and details of data analysis and the results are summarized in Section 8.2.

\subsection{Hardware Configuration}

In order to estimate the system bandwidth requirements, we first define the initial hardware configuration. This configuration describes the relationship between Pixel and SCT RODs and Data Formatter boards. The Data Formatter board and shelf assignments, as well as inter-shelf communication links are also taken into consideration. Since the data traffic inside of the Data Formatter System is expected to be largely dependent system configuration, we start with a configuration which we expect will minimize data volume on the backplane and inter-shelf links.

\subsubsection{ROD - FPGA - FTK Tower Assignment}

FPGA-FTK Tower Initial Assignment First we define the assignment of 64 FTK $\eta-\phi$ towers to the 64 FPGAs in four ATCA crates of the Data Formatter System. Table 2 shows the initial mapping. On the Table, " $\phi$ XX Y Z" indicates the FTK towers linked from the FPGAs. Refer Figure 2(a), 2(b) for FTK $\eta$ - $\phi$ tower partitioning, and the numbering scheme of $\phi$ sectors.

\begin{tabular}{l||c|c|c|c|c|c|c|c}
\hline Shelf0 & Board0 & Board1 & Board2 & Board3 & Board4 & Board5 & Board6 & Board7 \\
\hline Top FPGA & $\phi 00 \mathrm{C} \mathrm{E}$ & $\phi 00 \mathrm{C} \mathrm{B}$ & $\phi 00 \mathrm{~A} \mathrm{~B}$ & $\phi 00 \mathrm{~A} \mathrm{E}$ & $\phi 02 \mathrm{C} \mathrm{E}$ & $\phi 02 \mathrm{C} \mathrm{B}$ & $\phi 02 \mathrm{~A} \mathrm{~B}$ & $\phi 02 \mathrm{~A} \mathrm{E}$ \\
\hline Bottom FPGA & $\phi 01 \mathrm{C} \mathrm{E}$ & $\phi 01 \mathrm{C} \mathrm{B}$ & $\phi 01 \mathrm{~A} \mathrm{~B}$ & $\phi 01 \mathrm{~A} \mathrm{E}$ & $\phi 03 \mathrm{C} \mathrm{E}$ & $\phi 03 \mathrm{C} \mathrm{B}$ & $\phi 03 \mathrm{~A} \mathrm{~B}$ & $\phi 03 \mathrm{~A} \mathrm{E}$ \\
\hline
\end{tabular}

\begin{tabular}{l||c|c|c|c|c|c|c|c} 
Shelf1 & Board0 & Board1 & Board2 & Board3 & Board4 & Board5 & Board6 & Board7 \\
\hline Top FPGA & $\phi 04$ C E & $\phi 04$ C B & $\phi 04$ A B & $\phi 04$ A E & $\phi 06$ C E & $\phi 06$ C B & $\phi 06$ A B & $\phi 06$ A E \\
\hline Bottom FPGA & $\phi 05$ C E & $\phi 05$ C B & $\phi 05$ A B & $\phi 05$ A E & $\phi 07$ C E & $\phi 07$ C B & $\phi 07$ A B & $\phi 07$ A E \\
\hline
\end{tabular}

\begin{tabular}{l||c|c|c|c|c|c|c|c} 
Shelf2 & Board0 & Board1 & Board2 & Board3 & Board4 & Board5 & Board6 & Board7 \\
\hline Top FPGA & $\phi 08$ C E & $\phi 08$ C B & $\phi 08$ A B & $\phi 08$ A E & $\phi 10$ C E & $\phi 10$ C B & $\phi 10$ A B & $\phi 10$ A E \\
\hline Bottom FPGA & $\phi 09$ C E & $\phi 09$ C B & $\phi 09$ A B & $\phi 09$ A E & $\phi 11$ C E & $\phi 11$ C B & $\phi 11$ A B & $\phi 11$ A E \\
\hline
\end{tabular}

\begin{tabular}{l||c|c|c|c|c|c|c|c} 
Shelf3 & Board0 & Board1 & Board2 & Board3 & Board4 & Board5 & Board6 & Board7 \\
\hline Top FPGA & $\phi 12$ C E & $\phi 12$ C B & $\phi 12$ A B & $\phi 12$ A E & $\phi 14$ C E & $\phi 14$ C B & $\phi 14$ A B & $\phi 14$ A E \\
\hline Bottom FPGA & $\phi 13$ C E & $\phi 13$ C B & $\phi 13$ A B & $\phi 13$ A E & $\phi 15$ C E & $\phi 15$ C B & $\phi 15$ A B & $\phi 15$ A E \\
\hline
\end{tabular}

Table 2: FPGA assignment in the Data Formatter system. 64 FPGAs are mounted on 32 Data Formatter boards in 4 ATCA shelves. Each FPGA is linked to one FTK towers. The notation of " $\phi X X \mathrm{~A} / \mathrm{C} \mathrm{E} / \mathrm{B}$ " indicates the location of the corresponding FTK towers(see Table 18).

ROD - FPGA Mapping Next, we determine the optimal relationship between RODs (SLINK fibers) and Data Formatter FPGAs ( $\eta$ - $\phi$ towers). Refer to Appendix A for the details of mapping between SLINK fibers and silicon detector modules. The 2D pixel cluster finder 
algorithms are logic and resource intensive. In order to balance Mezzanine Card resources we limit the number of Pixel SLINK fibers to three per Data Formatter FPGA. Most FPGAs receive two Pixel SLINK fibers, and four FPGAs exceptionally is assigned to three Pixel fibers. The initial cabling between 222 RODs and Data Formatter FPGAs are determined for the bandwidth study. The full mapping lists are shown in Appendix H.

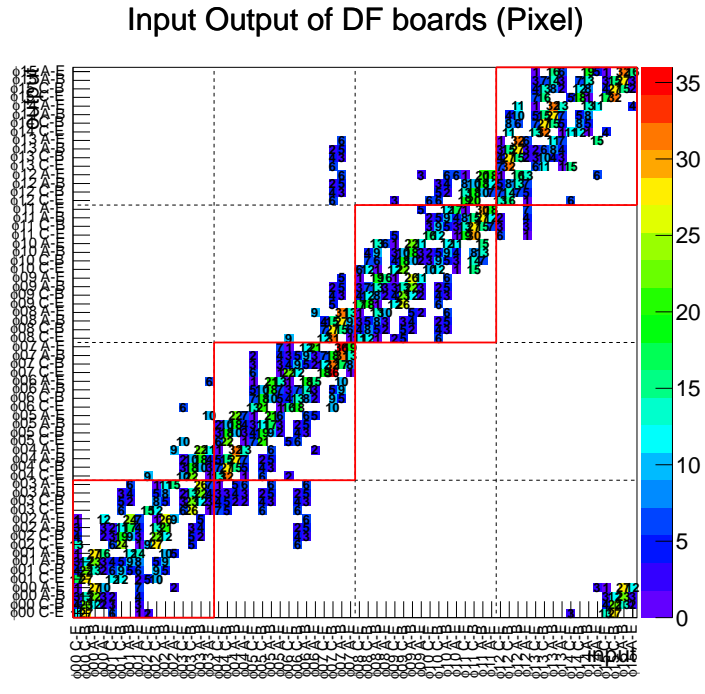

(a)

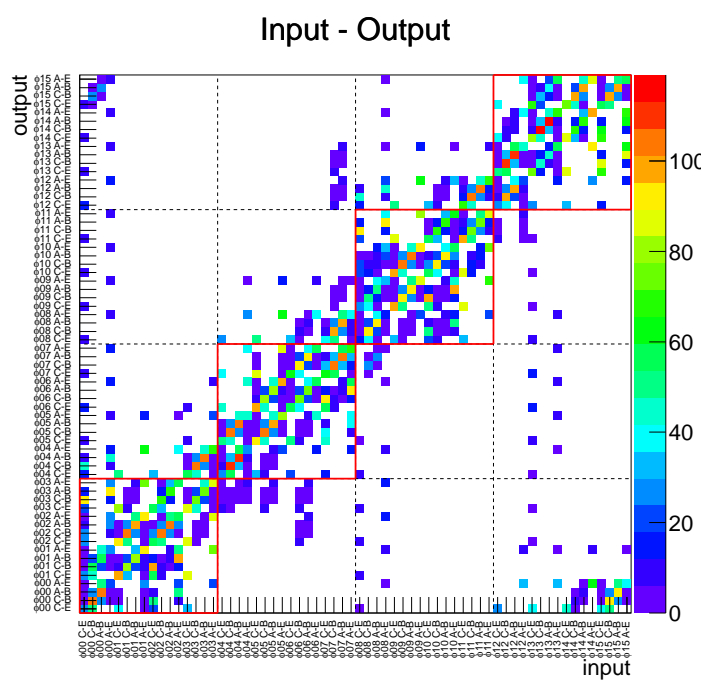

(c)

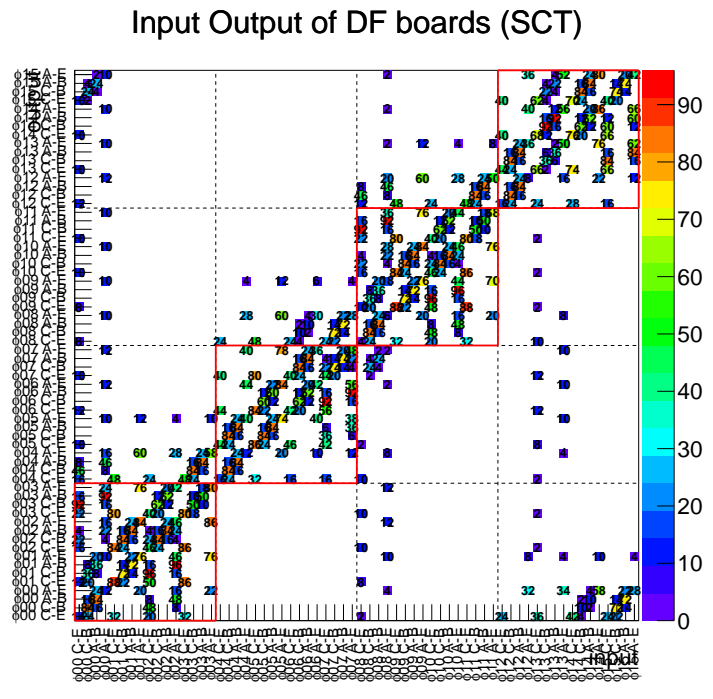

(b)

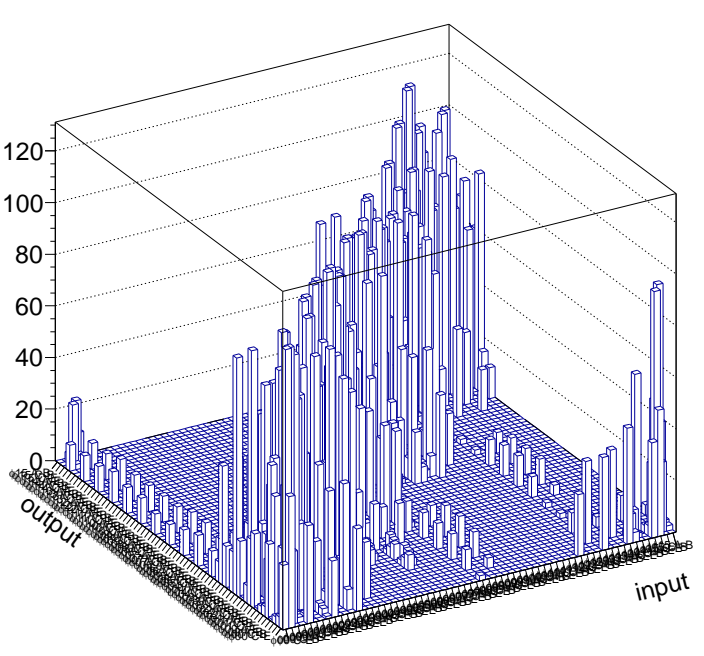

(d)

Figure 15: (a) and (b) show number of modules for source and destination FPGAs for Pixel and SCT, respectively. (c) and (d) show the sum of both Pixel and SCT.

Input and Output Correlation Figure 15 shows the data-traffic matrix inside of the Data Formatter System. The $x$-axis indicates the source FPGAs and the $y$-axis shows the destination FPGAs. Off-diagonal components, where the source FPGAs and the designation FPGAs are not the same, shows the data traffic in the Data Formatter System. Red squares in the figures represent ATCA shelf boundaries. Points outside these boxes represent data which must be shared between shelves via additional links which are discussed in the next section. 


\subsubsection{Inter-Shelf Communication}

Our optimized hardware configuration minimizes - but does not eliminate - data sharing between the four ATCA shelves. Fiber or copper links driven by the RTMs are be used for this purpose, which provides flexible external board-to-board communication beyond the ATCA shelves. Figure 16 shows how the inter-shelf links are connected.

\begin{tabular}{l} 
Shelf1 \\
\hline
\end{tabular} Phi Start

Figure 16: Direct fiber connection between two FPGAs for inter-shelf communication. Lines indicate bi-directional communication between two FPGAs.

\subsection{Data Analysis}

In the following sections we present the data-driven bandwidth requirement estimation for data paths in the Data Formatter System. In all cases we use actual collision data taken in 2012. This study is based on the Data Formatter System hardware configuration introduced in the previous sections.

First we explain the details of the input data volume from the silicon detectors (Section 8.2.3), and the total data volume in the output to the downstream FTK processors for all 64 FTK $\eta$ - $\phi$ towers (Section 8.2.4). Section 8.2.6 shows the expected data volumes in the three types of serial links in the Data Formatter System. In order to estimate the bandwidth requirement to maintain the data sharing at the highest expected luminosity environment of $\mathcal{L}=3 \times 10^{34} \mathrm{~cm}^{-2} \mathrm{~s}^{-1}$, the results are extrapolated according to the difference of number of pileup collisions, collision energy, and bunch spacing. Table 9, 10 summarizes the expected data volume and required bandwidth for the three types of Data Formatter serial links.

\subsubsection{Datasets}

We used about 10k collision events recorded in Muon Trigger stream with $\mathcal{L}=6.4 \times 10^{33} \mathrm{~cm}^{-2} \mathrm{~s}^{-1}$. The corresponding average of number of interaction per bunch crossing is $\langle\mu\rangle=30.0$. In order to extract the collision events, we select the events recorded at the collision bunch crossing according to the event BCID. Table 3 summarizes the data sample conditions.

\subsubsection{Number of Hits $\left(N_{\text {Pixel }}\right.$ and $\left.N_{\text {Strip }}\right)$}

The Pixel and SCT hit counts take the following factors into account: 


\begin{tabular}{|c|c|}
\hline Run Number & 214523 \\
\hline Lumi-block & 153 \\
\hline Number of events & 10,644 \\
\hline Data Stream & Muon Stream \\
\hline Collision Energy & $\sqrt{s}=8 \mathrm{TeV}$ \\
\hline Average number of interactions per bunch crossing & $\langle\mu\rangle=30.0$ \\
\hline Instantaneous luminosity & $\mathcal{L}=6.4 \times 10^{33} \mathrm{~cm}^{-2} \mathrm{~s}^{-1}$ \\
\hline Number of collision bunches at ATLAS & 1,368 \\
\hline Bunch Spacing & $50 \mathrm{~ns}$ \\
\hline SCT Readout mode & "X1X" timing cuts \\
\hline
\end{tabular}

Table 3: Data Sample summary. See the text for the SCT readout mode.

- No clustering is performed for both Pixel and SCT data. Normally clustering will be handled by FPGAs on the Mezzanine Cards. By ignoring the data reduction associated with clustering our bandwidth estimates are conservative.

- The " $01 \mathrm{X}$ " 3 timing requirement, also known as the SCT readout edge mode, is emulated in the data analysis while the SCT readout timing requirement was "X1X" in the run.

Note all the bandwidth requirements discussed in this section are based on this counting method for the Pixel and SCT hits. Appendix O provides more information about the number of hits expected when clustering is applied.

\subsubsection{Input Data Volume}

First, the input data volume is checked using the data sample of actual collision data with $\sqrt{s}=8 \mathrm{TeV},\langle\mu\rangle=30.0$, and $50 \mathrm{~ns}$ bunch spacing.

Figure 17 shows distributions of the numbers of hits per silicon module for different detector regions for Pixel and SCT, separately. Table 4 summarizes the average numbers of hits per module in each detector region.

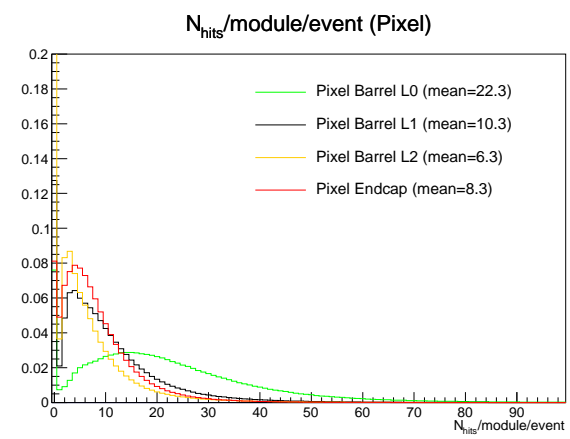

(a)

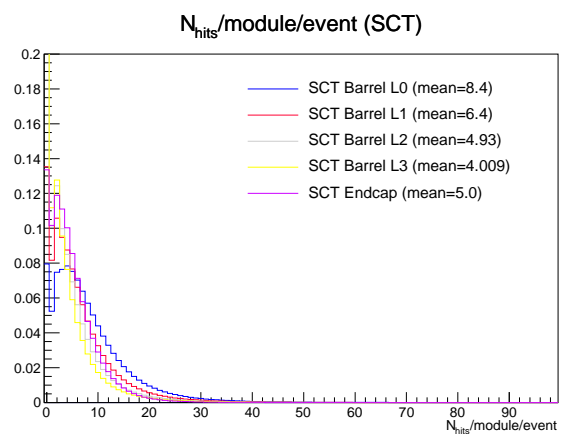

(b)

Figure 17: $N_{\text {hits }}$ per module per event for (a) Pixel and (b) SCT.

\footnotetext{
${ }^{3}$ These three characters represent three bunch crossings around the bunch crossing pointed to by the L1 trigger. These bunch crossings are called "previous", "central" and "next" in that order. "01X" stands for the requirement that the channel is active in the central bunch crossing with the middle " 1 " and inactive in the previous bunch crossing with the first " 0 ". The last "X" represents that we do not care about the next bunch crossing. Only the hits satisfying this timing requirement will be recorded.
} 


\begin{tabular}{l||c}
\hline & $\left\langle N_{\text {hits }} /\right.$ module/event $\rangle$ \\
\hline Pixel (Barrel L0) & 23 \\
\hline Pixel (Barrel L1) & 11 \\
\hline Pixel (Barrel L2) & 6.8 \\
\hline Pixel (Endcap) & 8.8 \\
\hline \hline SCT (Barrel L0) & $16 /$ module (8.2/layer of SCT doublet) \\
\hline SCT (Barrel L1) & $12 /$ module (6.2/layer of SCT doublet) \\
\hline SCT (Barrel L2) & $10 /$ module (4.8/layer of SCT doublet) \\
\hline SCT (Barrel L3) & $7.6 /$ module (3.8/layer of SCT doublet) \\
\hline SCT (Endcap) & $10 /$ module (5.0/layer of SCT doublet) \\
\hline
\end{tabular}

Table 4: Number of hits per module per event.

Figure 18 shows distributions of the numbers of hits per silicon module for different detector regions for Pixel and SCT, separately. Table 5 summarizes the average numbers of hits per module in each detector region.

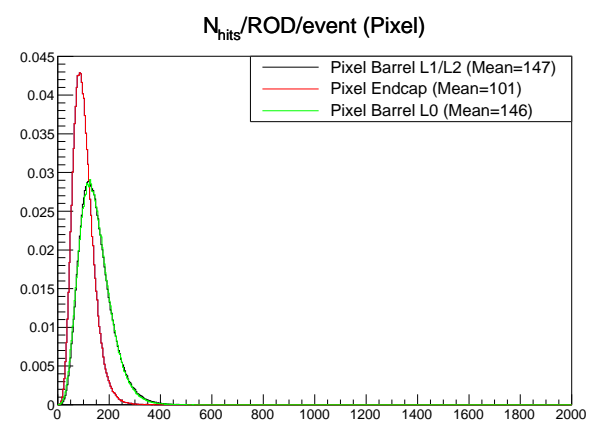

(a)

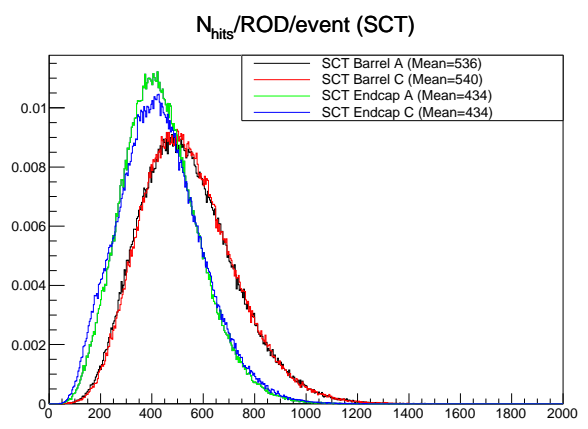

(b)

Figure 18: Observed number of hits per module per ROD. Note that exceptionally the " $01 \mathrm{X}$ " timing cut for SCT is not applied in the table.

\begin{tabular}{l||c|c}
\hline Region & $N_{\text {ROD }}$ & $\left\langle N_{\text {hits }} /\right.$ ROD $/$ event $\rangle$ \\
\hline Pixel Barrel (L0) & 44 & 146 \\
\hline Pixel Barrel (L1 or L2) & 64 & 147 \\
\hline Pixel Endcap & 24 & 101 \\
\hline SCT Barrel A & 22 & 536 \\
\hline SCT Barrel C & 22 & 540 \\
\hline SCT Endcap A & 23 & 434 \\
\hline SCT Endcap C & 23 & 434 \\
\hline
\end{tabular}

Table 5: First column shows number of RODs used for seven detector regions, and second column shows number of average number of hits per a ROD per event $\left(\left\langle N_{\text {hits }} / \mathrm{ROD} /\right.\right.$ event $\left.\rangle\right)$. 


\subsubsection{Output Data Volume}

In this section we show the number of hits sent downstream to FTK with $\sqrt{s}=8 \mathrm{TeV}$, $\langle\mu\rangle=30.0$, and $50 \mathrm{~ns}$ bunch spacing. Figure 21 shows the number of output hits from each detector region to the individual 64 FTK towers. (Refer to Table 18 for the 64 tower ID definitions.) The individual numbers for the Figure are summarized in Appendix K.

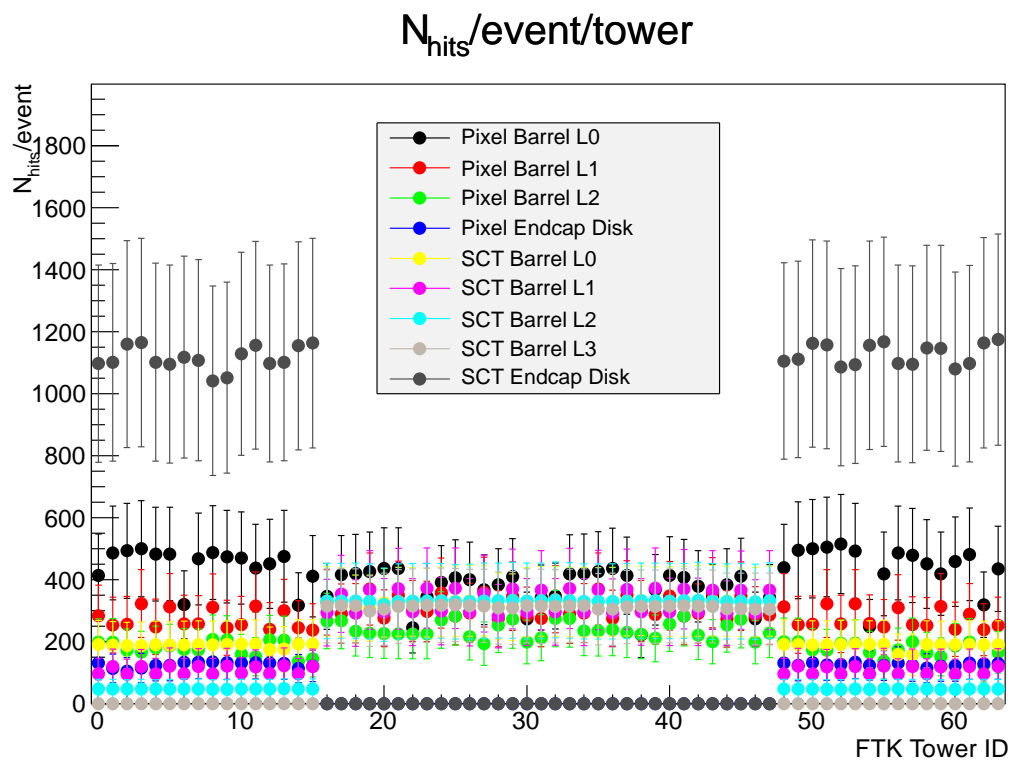

Figure 19: Summary of $N_{\text {hits }}$ to individual 64 FTK towers for each detector region.

The number of output $N_{\text {Pixel }}$ and $N_{\mathrm{SCT}}$ for all the 64 FTK towers are shown in Figure 21.

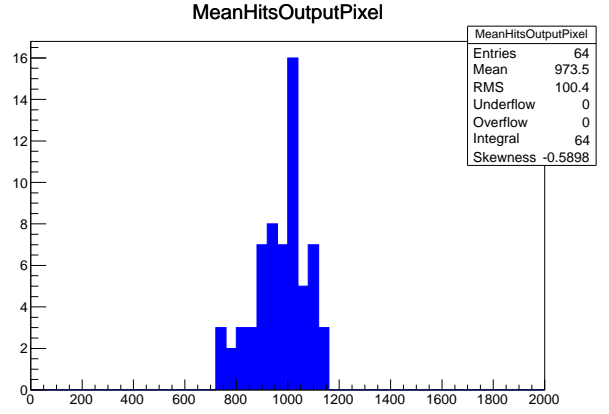

(a)

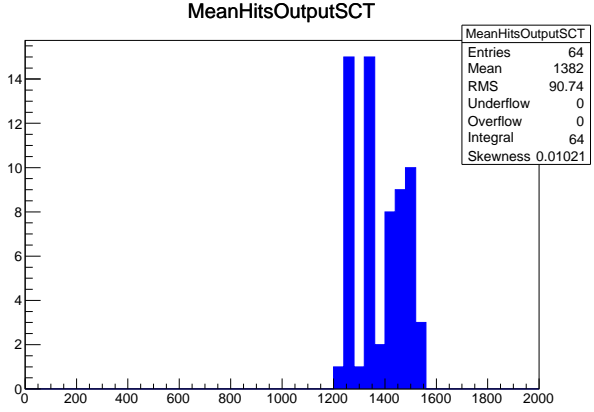

(b)

Figure 20: Summary of $N_{\text {hits }}$ to individual 64 FTK towers for each detector region for (a) $N_{\text {Pixel }}$, and (b) $N_{\text {SCT }}$. 
Up until this point we have considered the Data Formatter output as a single stream. In practice each Data Formatter FPGA drives multiple data links downstream to FTK, hence the number of transceivers on the RTM. The AUX Cards implement the first track fitting stage while the aptly-named Second Stage Boards (SSB) implement the second stage fitting (see Section 3.1). Figure 21 shows the $N_{\text {hits }}$ to the AUX Cards and SSB separately.

Note that that the AUX Card will receive all Pixel hits and SCT hits from five out of eight layers. The Second Stage Boards will receive the remaining three SCT layers. Therefore $N_{\text {hitsAuX }}$ is counted as " $N_{\text {Pixelhits }}+5 / 8 \times N_{\text {SCThits" }}$ " event-by event, and $N_{\text {hitsSCT }}$ is counted as " $3 / 8 \times N_{\text {hits" }}$ as approximation. Table 7 summarize the expectation of the typical data traffic among the 64 FTK towers and the maximum case for output $N_{\text {Pixel }}$, output $N_{\mathrm{SCT}}$, output $N_{\mathrm{AUX}}$, and output $N_{\mathrm{SSB}}$, respectively.

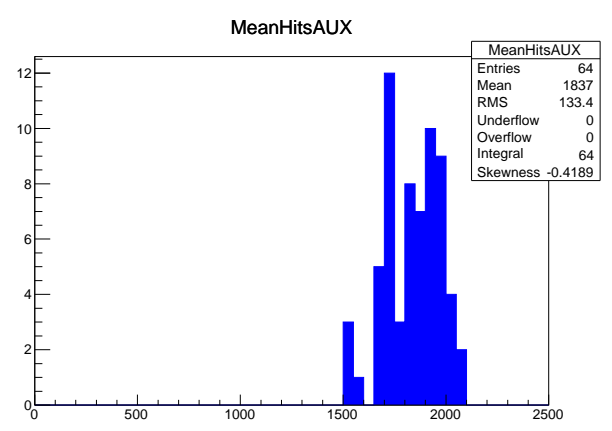

(a)

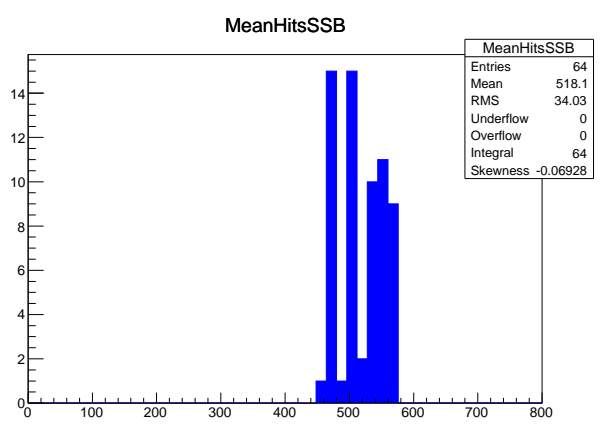

(b)

Figure 21: Summary of $N_{\text {hits }}$ to individual 64 AUXs and SSBs for each detector region.

\begin{tabular}{|c|c|c|}
\hline & average of $\left\langle N_{\text {hits }}\right\rangle$ & maximum of $\left\langle N_{\text {hits }}\right\rangle$ \\
\hline Total (output stream) & $2.4 \mathrm{e}+03$ & $2.6 \mathrm{e}+03$ \\
\hline Pixel Total (output stream) & $9.7 \mathrm{e}+02$ & $1.1 \mathrm{e}+03$ \\
SCT Total (output stream) & $1.4 \mathrm{e}+03$ & $1.5 \mathrm{e}+03$ \\
\hline AUX & $1.8 \mathrm{e}+03$ & $2.1 \mathrm{e}+03$ \\
SSB & $5.2 \mathrm{e}+02$ & $5.7 \mathrm{e}+02$ \\
\hline
\end{tabular}

Table 6: Summary of $N_{\text {hits }}$ sent to the downstream from the DF system. Typical data traffic for 64 FTK $\eta$ - $\phi$ towers and the maximum case are summarized for output $N_{\text {Pixel }}$, output $N_{\mathrm{SCT}}$, output $N_{\mathrm{AUX}}$, and output $N_{\mathrm{SSB}}$, respectively.

\subsubsection{Data Flow Routing Rules}

We simulate the number of hits transferred inside of the Data Formatter System to estimate data traffic. We have developed software to simulate the data traffic with the following routing rules:

1. Does the hit need to go to another shelf? If so, the hit will be sent over the inter-shelf link. If the hit arrived at the destination FPGA then, go to 4 , otherwise go to next 2.

2. Does the hit need to go to other FPGA on the board? If so, the hit will be sent over the local bus. If the hit arrived at the destination FPGA then, go to 4, otherwise go to next 3 . 
3. Does the hit need to go to another FPGA in the same shelf? If so, the hits will be sent over the backplane Fabric Interface to the destination board. The hit should arrive at the destination FPGA at this step, and go to next 4.

4. Send the data downstream to FTK.

The data flow model described here enables us to count the number of hits passing through individual serial links implemented in the Data Formatter System. The corresponding flowchart for the simulation is shown in Appendix L.

\subsubsection{Expected Number of Hits}

Our software simulation model enables us to run data records through the Data Formatter system on an event-by-event basis as we evaluate the number of hits transferred on each serial link. The typical $N_{\text {hits }}$ for the individual links are summarized in Figure 22 for (a) local bus communication, (b) ATCA Fabric Interface communication, and (c) inter-shelf communication, respectively. Most links in the system are utilized. The number of active links are:

- 64 out of 64 inter-FPGA local bus

- 409 out of 448 ATCA backplane Fabric Interface links

- 45 out of 64 inter-shelf links

Our data flow routing algorithms select the "most direct" route between FPGAs. In some cases this methodology results in links which are either unused or under-utilized, as is shown in the broad distribution in Figure 22. This suggests that by tuning the routing algorithms we should be able to divert traffic away from high volume links and take advantage of the available bandwidth on low-volume links.

\begin{tabular}{|c|c|c|}
\hline & average of $\langle N\rangle$ & maximum of $\langle N\rangle$ \\
\hline Fabric & $2.6 \mathrm{e}+02$ & $6.3 \mathrm{e}+02$ \\
Local Bus & $1.0 \mathrm{e}+03$ & $1.7 \mathrm{e}+03$ \\
Inter-Crate & $6.0 \mathrm{e}+02$ & $1.3 \mathrm{e}+03$ \\
\hline
\end{tabular}

Table 7: Summary of $N_{\text {hits }}$ transferred in the DF system. Typical data traffic and the maximum case are summarized for each Data Formatter link type. The typical value is average of the $\langle N\rangle$ for all the active links. This data set was collected under the following conditions: $\sqrt{s}=8 \mathrm{TeV},\langle\mu\rangle=30.0$, with $50 \mathrm{~ns}$ bunch spacing.

\subsubsection{Bandwidth Requirements}

To estimate the data bandwidth requirements for system links we must first convert the number of hits $N_{\text {hits }}$ into 32 -bit words $N_{\text {words }}$. The Data Formatter FPGAs do not see the incoming SLINK data records, but rather they see the output of the cluster finder Mezzanine Cards. This output format is shown in Figure 23.

Using the Mezzanine Card format, we can approximate the number of 32-bit words using the formula $N_{\text {words }} \sim N_{\text {Pixel Hits }}+0.5 \times N_{\text {SCT hits }}+N_{\text {Pixel module }}+N_{\text {SCT module }}$. (Our word count also takes into account the data format header words, which is why $N_{\text {Pixel module }}+N_{\mathrm{SCT}}$ module are included in the formula.) The $N_{\text {hits }}$ analysis is discussed in Section 8.2.4 and Section 8.2.6 will be translated into $N_{\text {words }}$ as summarized in Figure 24 and Figure 25 . The typical $N_{\text {words }}$ and the maximum case are summarized in Table 8 . 

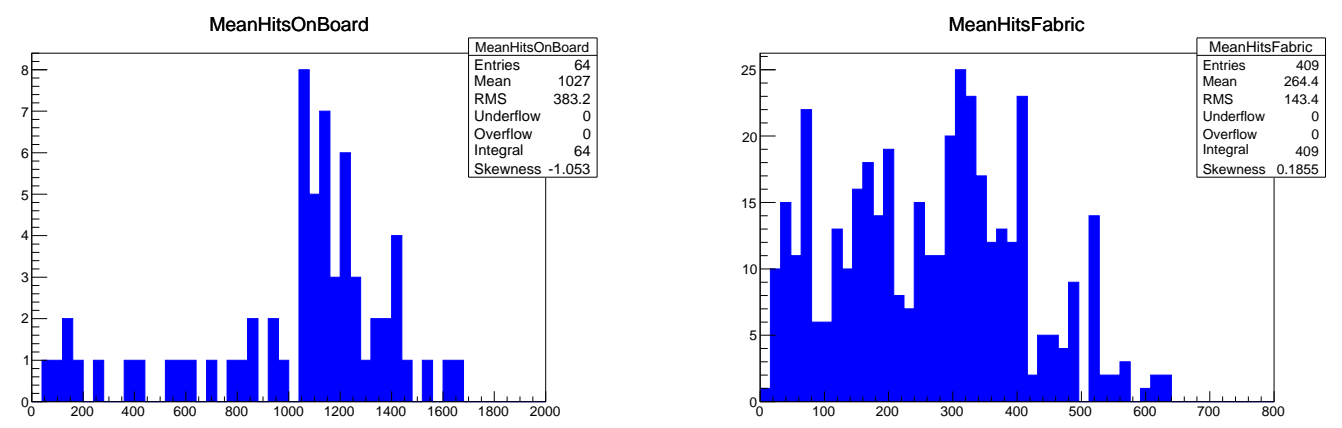

(a)

(b)

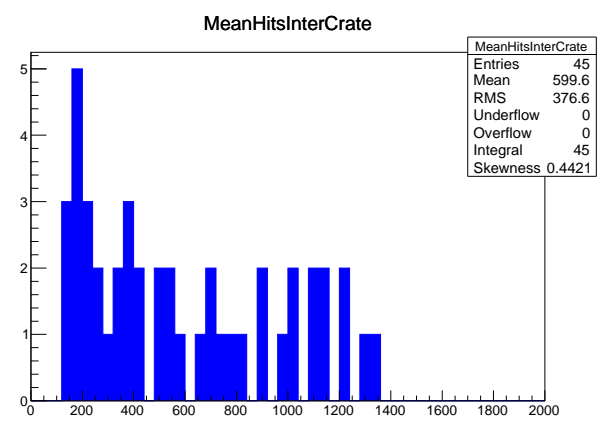

(c)

Figure 22: Summary of sum of $N_{\text {Pixel }}$ and $N_{\text {SCT }}$ transferred in the DF system. (a) shows data volume transferred via the so-called inter-FPGA link connecting two FPGAs on a board. (b) shows data volume transferred via the so-called fabric link supported by the ATCA backplane connecting two FPGAs on the different board in the same ATCA shelf. (c) shows data volume transferred via the so-called inter-Shelf link connecting two FPGAs via optical fibers driven by the RTM. The connectivity via this link is totally flexible. See the text for more discussion.

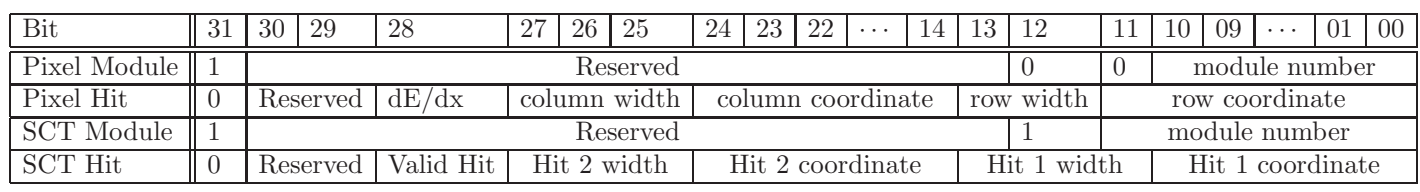

Figure 23: Input data format

\subsubsection{Extrapolation to Target LHC Operating Conditions}

In order to extrapolate from the reference run $(\sqrt{s}=8 \mathrm{TeV},\langle\mu\rangle=30.0$, and 50 ns bunch spacing) into target condition with $\sqrt{s}=14 \mathrm{TeV},\langle\mu\rangle=70.0$, and $25 \mathrm{~ns}$ bunch spacing, the data volume dependence on the $\langle\mu\rangle$ is studied.

$\langle\mu\rangle$ Difference To confirm the linearity with the real collision data up to $\langle\mu\rangle=70$, we analyzed the special runs with $\langle\mu\rangle$ taken in July 2012 as well. For this study, we used collision events triggered by a random trigger in the minimum bias stream to get rid of the trigger 


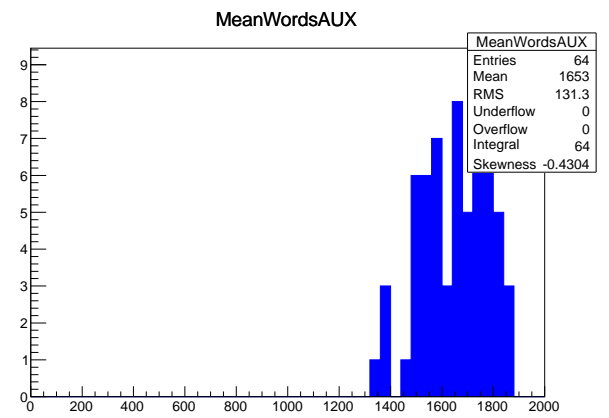

(a)

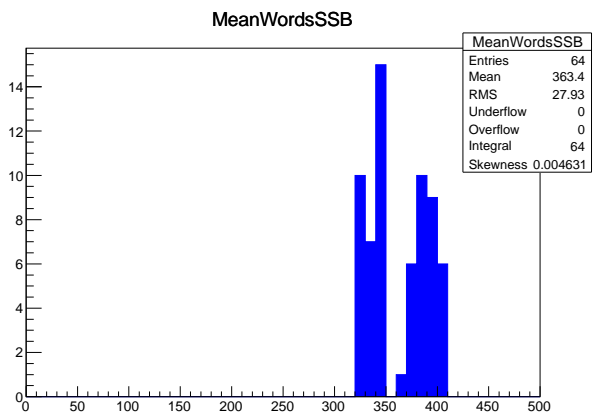

(b)

Figure 24: Summary of $N_{\text {words }}$ to individual 64 AUXs and SSBs for each detector region, corresponding to Figure!24. This data set was collected under the following conditions: $\sqrt{s}=$ $8 \mathrm{TeV},\langle\mu\rangle=30.0$, and $50 \mathrm{~ns}$ bunch spacing.

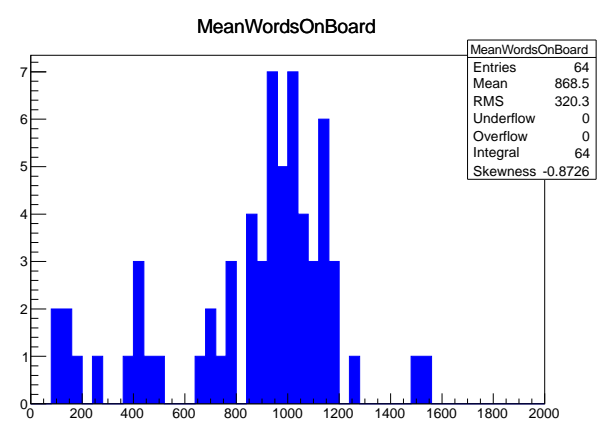

(a)

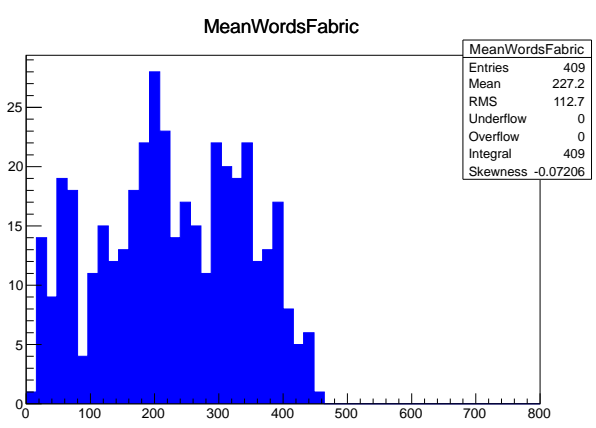

(b)

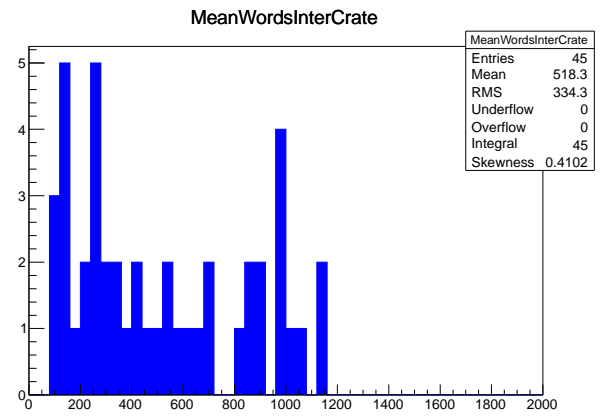

(c)

Figure 25: Summary of $N_{\text {words }}$ transferred in the DF system, corresponding to Figure 22. This data set was collected under the following conditions:t $\sqrt{s}=8 \mathrm{TeV},\langle\mu\rangle=30.0$, and 50 ns bunch spacing.

configuration difference between run periods. The linearity as a function of $\langle\mu\rangle$ is shown up to $\langle\mu\rangle=70$ for both Pixel and SCT detectors. Based on this observation, we scale up the 


\begin{tabular}{|c|c|c|}
\hline & average of $\langle N\rangle$ & maximum of $\langle N\rangle$ \\
\hline AUX & $1.7 \mathrm{e}+03$ & $1.9 \mathrm{e}+03$ \\
SSB & $3.6 \mathrm{e}+02$ & $4.1 \mathrm{e}+02$ \\
\hline Fabric & $2.3 \mathrm{e}+02$ & $4.6 \mathrm{e}+02$ \\
Local Bus & $8.7 \mathrm{e}+02$ & $1.5 \mathrm{e}+03$ \\
Inter-Crate & $5.2 \mathrm{e}+02$ & $1.1 \mathrm{e}+03$ \\
\hline
\end{tabular}

Table 8: Summary of $N_{\text {words }}$ for each type of links with the definition of encoding shown in Figure 23. This data was collected under the following conditions: $\sqrt{s}=8 \mathrm{TeV},\langle\mu\rangle=30.0$, with a 50 ns bunch spacing.

number of hits to extrapolate the data volume from $\langle\mu\rangle=30$ up to $\langle\mu\rangle=70$. More discussion is shown in Appendix M.

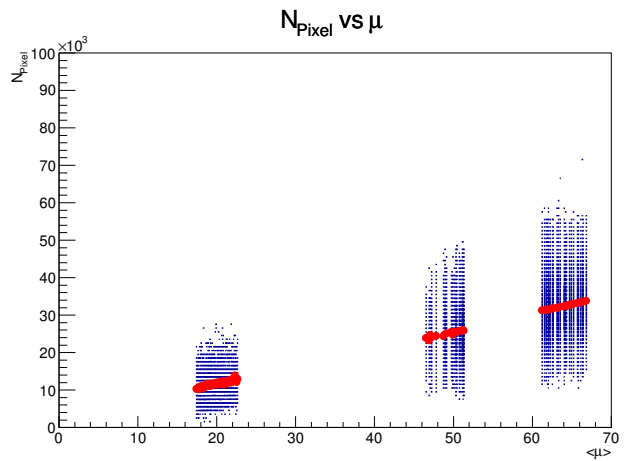

(a)

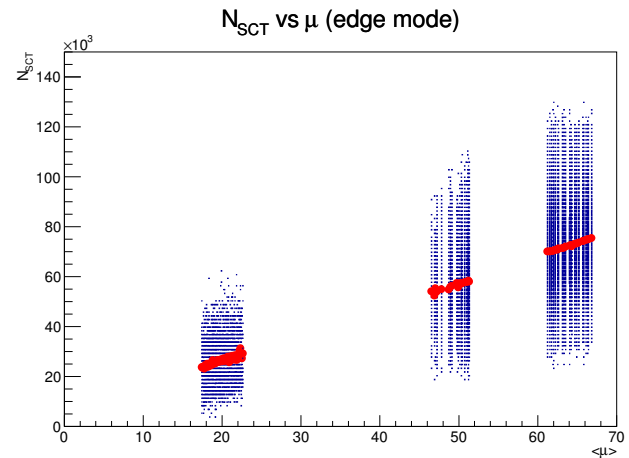

(b)

Figure 26: Data volume dependence on $\langle\mu\rangle$ with $\sqrt{s}=8 \mathrm{TeV}$. (a) Number of Pixel raw hits, and (b) Number of SCT raw hits passing "01X" timing cut (emulating "edge mode" operation).

Energy and Bunch-Spacing Difference To understand the difference between two conditions:

- $\sqrt{s}=8 \mathrm{TeV}$ and $50 \mathrm{~ns}$ bunch spacing

- $\sqrt{s}=14 \mathrm{TeV}$ and $25 \mathrm{~ns}$ bunch spacing

the two MC samples are compared as shown in the Figure 27 for Pixel detector behavior ${ }^{4}$. By comparison between $\sqrt{s}=14 \mathrm{TeV}$ samples and extrapolated point of $\sqrt{s}=8 \mathrm{TeV}$ samples, we extracted roughly $45 \%$ increase for the $\sqrt{s}=14 \mathrm{TeV}$ and $25 \mathrm{~ns}$ bunch spacing. Then this $45 \%$ increase of the $N_{\text {hits }}$ applied for for both Pixel and SCT number of hits extrapolation for the energy and bunch crossing difference.

Further discussion for the MC-Data comparison study are shown in Appendix N.

\subsubsection{Bandwidth Requirement Summary}

Taking the difference of 1) luminosity, 2) beam energy, and 3) bunch-crossing, a scale factor of about 3.4 is applied to the hit count ${ }^{5}$, and the $N_{\text {words }}$ is scaled up accordingly to estimate operating with $\sqrt{s}=14 \mathrm{TeV},\langle\mu\rangle=70.0$, and $25 \mathrm{~ns}$ bunch spacing.

\footnotetext{
${ }^{4} \mathrm{MC}$ comparison for SCT was not done so far due to the difference of the readout configuration between the two samples. The "X1X" cut is pre-applied to $50 \mathrm{~ns}$ bunch spacing runs, and " $01 \mathrm{X}$ " cut is pre-applied to $25 \mathrm{~ns}$ bunch spacing runs.

${ }^{5}$ For pileup multiplicity difference $70 . / 30$. In total $70 . / 30 \times 1.45 \sim 3.4$
} 


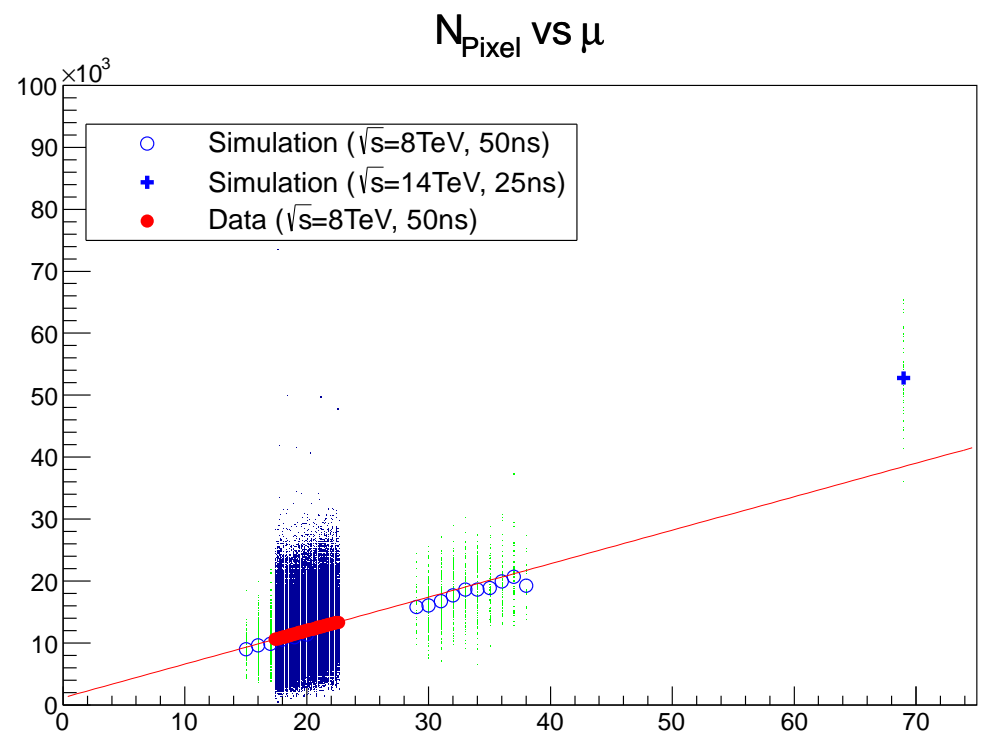

Figure 27: Number of Pixel hits as a function of $\langle\mu\rangle$. Two different MC samples are plotted for $14 \mathrm{TeV}$ and $8 \mathrm{TeV}$. Just for reference, the data in 2012 are also superimposed.

Then the number of words are translated into number of bits. Each word is 32-bits as shown in Figure 23. Since these data words will be sent over high speed serial links we must also take into account the overhead associated with 8b/10b encoding. Thus each 32-bit data word will be transmitted as 40 bits.

Table 9 and Table 10 summarize the expected $N_{\text {hits }}$ and $N_{\text {words }}$ under target LHC operating conditions. Both the typical value and the maximum case are shown in this table. The maximum $N_{\text {words }}$ determines the bandwidth requirement for each link, which is shown in the third column of Table 10. The rightmost column shows the system bandwidth capacity.

\begin{tabular}{|c|c|c|}
\hline & average of $\langle N\rangle$ & maximum of $\langle N\rangle$ \\
\hline Total (output stream) & $8.0 \mathrm{e}+03$ & $8.8 \mathrm{e}+03$ \\
\hline Pixel Total (output stream) & $3.3 \mathrm{e}+03$ & $3.8 \mathrm{e}+03$ \\
SCT Total (output stream) & $4.7 \mathrm{e}+03$ & $5.2 \mathrm{e}+03$ \\
\hline AUX & $6.2 \mathrm{e}+03$ & $7.1 \mathrm{e}+03$ \\
SSB & $1.8 \mathrm{e}+03$ & $2.0 \mathrm{e}+03$ \\
\hline Fabric & $9.0 \mathrm{e}+02$ & $2.2 \mathrm{e}+03$ \\
Local Bus & $3.5 \mathrm{e}+03$ & $5.6 \mathrm{e}+03$ \\
Inter-Crate & $2.0 \mathrm{e}+03$ & $4.4 \mathrm{e}+03$ \\
\hline
\end{tabular}

Table 9: Summary of the expected $N_{\text {hits }}$ with $\sqrt{s}=14 \mathrm{TeV},\langle\mu\rangle=70.0$, and 25 ns bunch spacing. These numbers have been extrapolated as discussed in Section 8.2.8. 


\begin{tabular}{|c|c|c|c||c|}
\hline & average of $\langle N\rangle$ & maximum of $\langle N\rangle$ & BW Required & Link Capacity \\
\hline Total (output stream) & $5.9 \mathrm{e}+03$ & $6.6 \mathrm{e}+03$ & - & - \\
\hline Pixel Total (output stream) & $3.4 \mathrm{e}+03$ & $3.9 \mathrm{e}+03$ & - & - \\
SCT Total (output stream) & $2.6 \mathrm{e}+03$ & $2.8 \mathrm{e}+03$ & - & - \\
\hline AUX & $5.0 \mathrm{e}+03$ & $5.6 \mathrm{e}+03$ & 22.6 & 48 \\
SSB & $9.6 \mathrm{e}+02$ & $1.1 \mathrm{e}+03$ & 4.31 & 6.0 \\
\hline Fabric & $6.7 \mathrm{e}+02$ & $1.4 \mathrm{e}+03$ & 5.73 & 10 \\
Local Bus & $2.5 \mathrm{e}+03$ & $4.7 \mathrm{e}+03$ & 18.7 & 24 \\
Inter-Crate & $1.5 \mathrm{e}+03$ & $3.5 \mathrm{e}+03$ & 13.8 & 20 \\
\hline
\end{tabular}

Table 10: Summary of the expected $N_{\text {words }}$ at $\sqrt{s}=14 \mathrm{TeV},\langle\mu\rangle=70.0$, and 25 ns bunch spacing. These values have been extrapolated as discussed in Section 8.2.8. The third column shows the maximum worst-case link bandwidth requirements (in Gb/s). The forth column shows the preliminary system bandwidth capacity (in $\mathrm{Gb} / \mathrm{s}$ ) with the current design.

\subsection{Conclusion}

In order to study the bandwidth requirements of the Data Formatter system we have first defined what we consider is the optimal hardware configuration of RODs, SLINKs, Data Formatter boards, and ATCA shelves based on our study. Real event data records have been run through a simulation model of the entire Data Formatter system and the number of hits flowing through each link have been analyzed. These results have been scaled and extrapolated out to the target LHC operating conditions of $\sqrt{s}=14 \mathrm{TeV},\langle\mu\rangle=70.0$, and 25 ns bunch spacing. Under these target operating conditions the Data Formatter design meets bandwidth requirements for all internal data links. The IBL data volume case will be added in future after fixing all the hardware configuration of IBL detectors.

Given the Data Formatter flexible design, there exists significant reserve capacity in the system. The full mesh ATCA backplane makes possible many equivalent alternate paths between FPGAs. Data may be diverted from high-volume links to under-utilized links, effectively reducing the bandwidth requirements while at the same time improving system latency. These studies, as well as their impact on the FPGA data routing engine firmware, are ongoing. 


\section{Appendix A System Analysis}

The ATLAS detector and readout electronics were not originally designed to support a track trigger. Specifically, the physical locations of the inner detector modules do not line up with symmetric $\phi$ region boundaries. Furthermore, the mapping between modules and RODs introduces another level of asymmetry into the readout scheme. Early on we recognized that these factors would prove to be the primary challenge faced in designing the Data Formatter. In this section we present a summary of our early analysis.

\section{A.1 Pixel and SCT readout partitioning}

The Data Formatter system receives silicon hits via SLINK fibers from the Pixel and SCT RODs. Table 11 and Table 12 summarize the arrangement of the ROD modules for Pixel and SCT. The RODs are connected to the upstream silicon detector modules over optic fiber links. Table 1314 summarize the number of detector modules for Pixel and SCT. In total 222 RODs modules are used to read out hit data from 1,744 Pixel modules, 4,224 SCT barrel modules, and 3,952 SCT end-cap modules. Our early simulation results [1] indicated that the amount of data traffic between Data Formatter boards is highly dependent on the module-ROD mapping, and Figure 28293031 show the module-ROD mapping for the current existing detectors in $x-y$ view. Numbers in the figures indicate "ROD IDs" assigned to the modules. (The ROD ID is explained in the Appendix F.) It is important to note that module-ROD mapping is optimized to balance data traffic over the optic fiber links and to use the bandwidth resources most effectively; the mapping was never intended to organize hits into the uniform symmetric $\eta$ - $\phi$ towers that a tracking trigger system requires.

This study shows that some RODs cover a significant $\phi$ regions or even non-contiguous detector regions. The following are examples of such exceptions. Figure 28(c) shows each ROD covers $1 / 4$ of the ring in the second disk of Endcap Pixel (as an example of large $\phi$ coverage). Figure 28(a) shows a ROD that covers two separate regions in the second layer of Pixel Barrel (as an example of the separate coverage). The RODs are the "worst case" configurations and result in maximum data sharing in the Data Formatter system.

\begin{tabular}{l||c|c|c|c}
\hline & Barrel - A & Barrel - C & Endcap - A & Endcap - C \\
\hline Layer/Disk 0 & 22 & 22 & 8 & 8 \\
\hline Layer/Disk 1 & 19 & 19 & 4 & 4 \\
\hline Layer/Disk 2 & 13 & 13 & - & - \\
\hline \hline total & 54 & 54 & 12 & 12 \\
\hline
\end{tabular}

Table 11: Number of RODs for Pixel readout. RODs for Endcap Disk0 cover Endcap Disk2 as well. 132 RODs are used in total.

\begin{tabular}{c|c|c|c}
\hline Barrel - A & Barrel - C & Endcap - A & Endcap - C \\
\hline 22 & 22 & 23 & 23 \\
\hline
\end{tabular}

Table 12: Number of RODs for SCT readout. 90 RODs are used in total. The SCT RODs boundary does not correspond to the layer/disk (See Figure 30 and Figure 31), and therefore only the sum for all the layers/disks are shown in the Table, differently from case of Pixel. 


\begin{tabular}{l||c|c|c|c}
\hline & Barrel - A & Barrel - C & Endcap - A & Endcap - C \\
\hline Layer/Disk 0 & 143 & 143 & 48 & 48 \\
\hline Layer/Disk 1 & 247 & 247 & 48 & 48 \\
\hline Layer/Disk 2 & 338 & 338 & 48 & 48 \\
\hline \hline total & 728 & 728 & 144 & 144 \\
\hline
\end{tabular}

Table 13: Number of modules for Pixel readout. The numbers shown in the table are identical to those of input fibers to 132 Pixel RODs. 1744 modules are used in total.

\begin{tabular}{l||c|c|c|c}
\hline & Barrel - A & Barrel - C & Endcap - A & Endcap - C \\
\hline Layer/Disk 0 & 384 & 384 & 184 & 184 \\
\hline Layer/Disk 1 & 480 & 480 & 264 & 264 \\
\hline Layer/Disk 2 & 576 & 576 & 264 & 264 \\
\hline Layer/Disk 3 & 672 & 672 & 264 & 264 \\
\hline Disk 4 & - & - & 264 & 264 \\
\hline Disk 5 & - & - & 264 & 264 \\
\hline Disk 6 & - & - & 184 & 184 \\
\hline Disk 7 & - & - & 184 & 184 \\
\hline Disk 8 & - & - & 104 & 104 \\
\hline \hline total & 2112 & 2112 & 1976 & 1976 \\
\hline
\end{tabular}

Table 14: Number of modules for SCT readout. Note that SCT doublet layers are counted individually, and the numbers shown in the table are identical to those of input fibers to 90 SCT RODs. 4088 modules (8176 layers) are used in total.

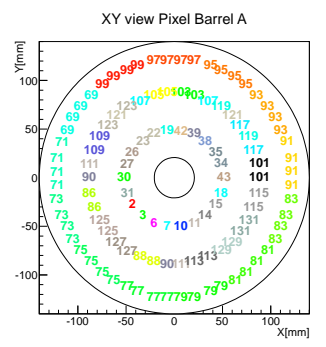

(a)

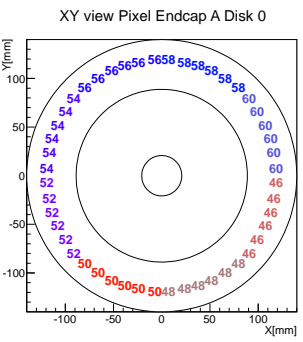

(b)

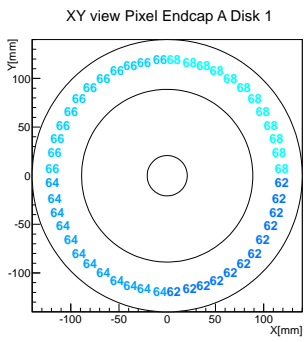

(c)

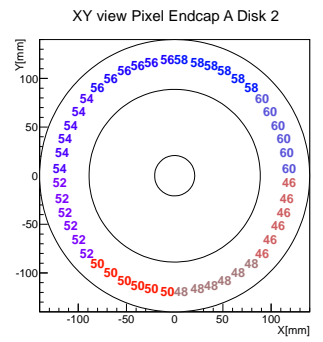

(d)

Figure 28: (a) Pixel A Barrel (3 layers), (b) (c) (d) Pixel A Endcap 3 Disks.

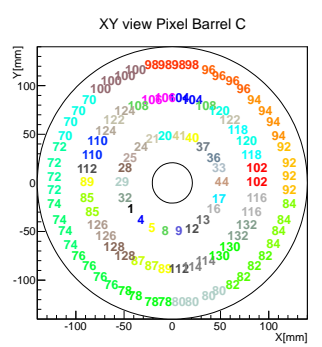

(a)

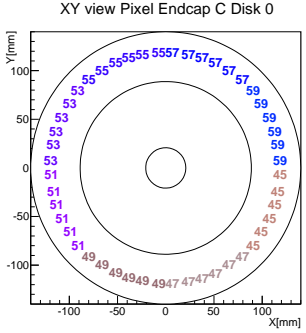

(b)

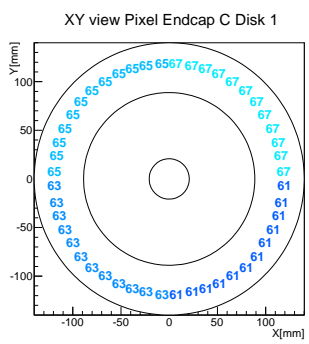

(c)

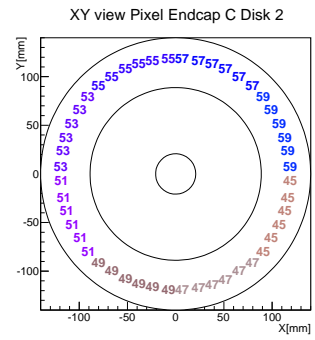

(d)

Figure 29: The RODs coverage for Pixel modules in C-sides are shown in $x$ - $y$ plane. (a) Pixel C Barrel (3 layers), (b) (c) (d) Pixel C Endcap 3 Disks. 


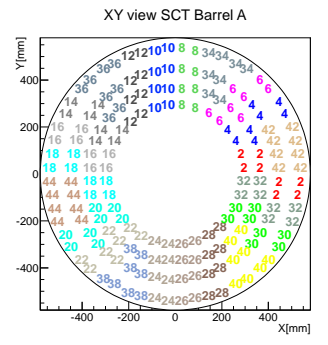

(a)

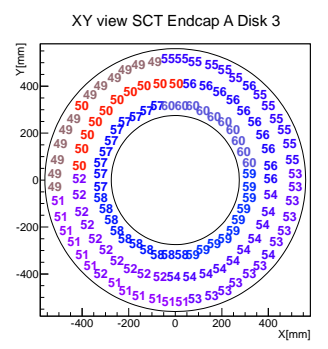

(e)

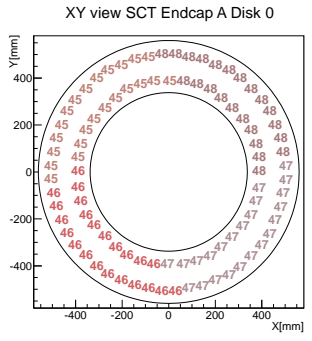

(b)

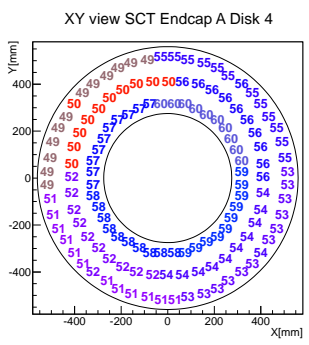

(f)

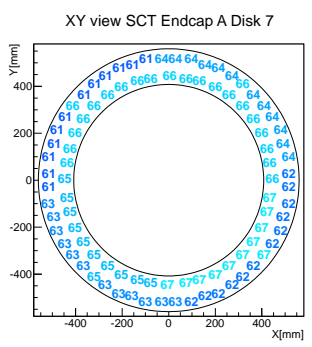

(i)

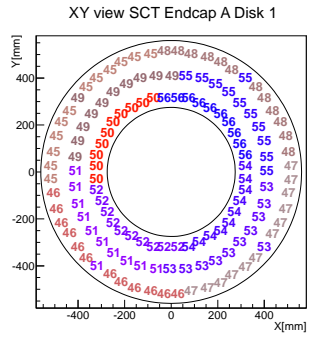

(c)

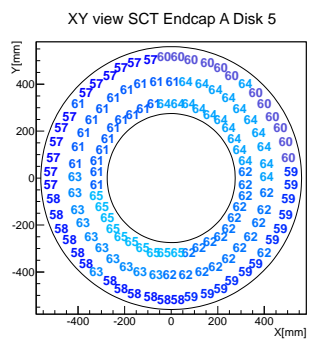

(g)

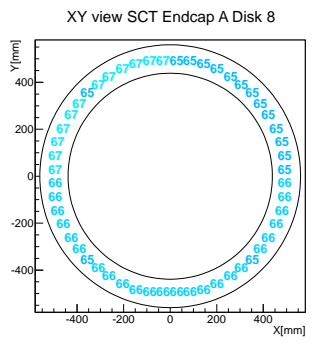

(j)

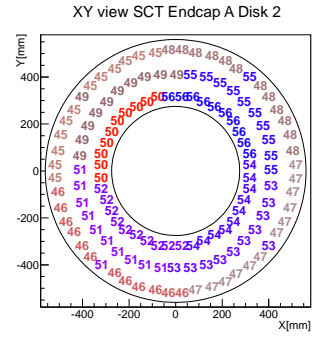

(d)

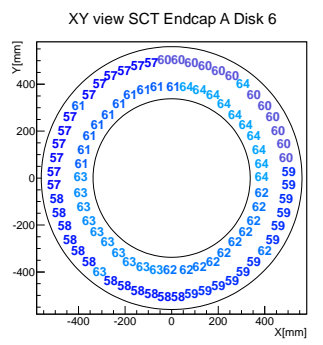

(h)

Figure 30: (a) SCT A Barrel (4 lures), (b) - (J) SCT A Endcap 9 Disks. 


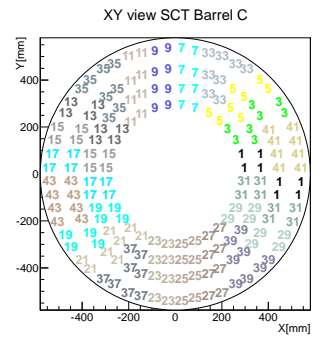

(a)

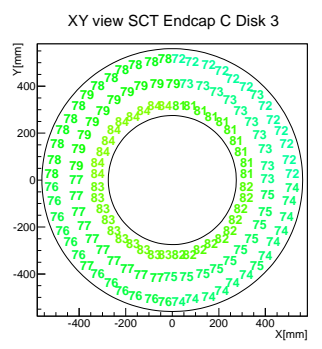

(e)

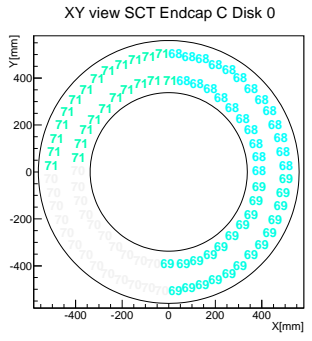

(b)

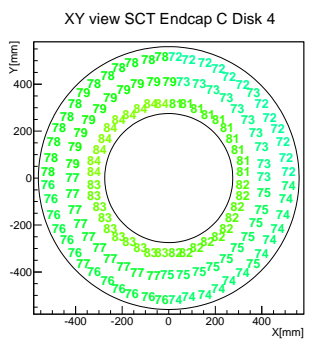

(f)

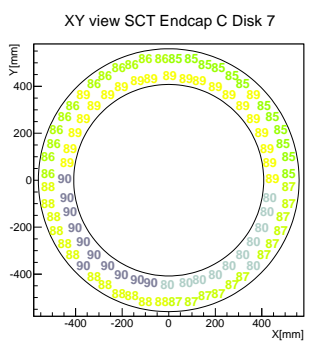

(i)

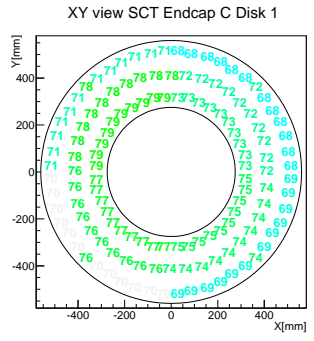

(c)

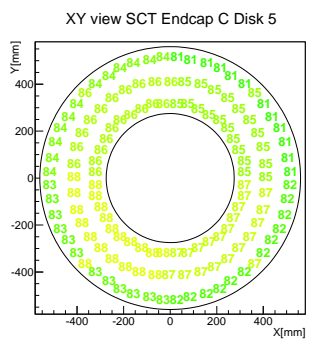

(g)

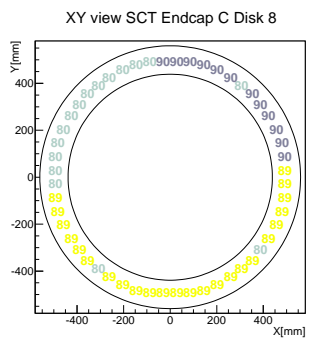

(j)

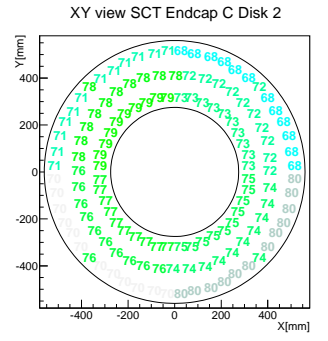

(d)

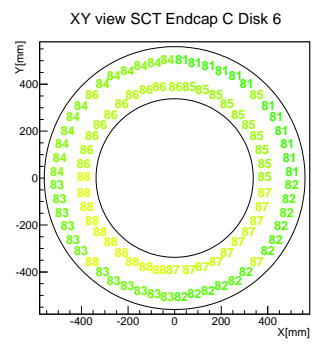

(h)

Figure 31: (a) SCT C Barrel (4 layers), (b) - (J) SCT C Endcap 9 Disks. 


\section{A.2 FTK $\eta$ - $\phi$ Tower Partitioning}

The FTK system divides the detector volume into 64 symmetric sub-regions according to the $(\eta, \phi)$ of the silicon module (Figures $2(\mathrm{a})$ and $2(\mathrm{~b})$ ).

In $\phi$ the detector is divided into 16 sectors of $360^{\circ} / 16=22.5^{\circ}$ with $10^{\circ}$ overlap. Table A.2 describes the FTK $\phi$ sector boundaries (refer to Figure 2(a) for the sector numbering convention). The overlap is designed for variation of curvature for $p_{T}>1 \mathrm{GeV}$, and multiple scattering.

\begin{tabular}{|l|l|l|l|l|l|l|l|l|}
\hline FTK $\phi$ Sector & 0 & 1 & 2 & 3 & 4 & 5 & 6 & 7 \\
\hline Start $\phi$ & $-22.5^{\circ}$ & $0^{\circ}$ & $22.5^{\circ}$ & $45^{\circ}$ & $67.5^{\circ}$ & $90^{\circ}$ & $112.5^{\circ}$ & $135^{\circ}$ \\
End $\phi$ & $10^{\circ}$ & $32.5^{\circ}$ & $55^{\circ}$ & $77.5^{\circ}$ & $100^{\circ}$ & $122.5^{\circ}$ & $145^{\circ}$ & $167.5^{\circ}$ \\
\hline
\end{tabular}

\begin{tabular}{|l|l|l|l|l|l|l|l|l|}
\hline FTK $\phi$ Sector & 8 & 9 & 10 & 11 & 12 & 13 & 14 & 15 \\
\hline Start $\phi$ & $157.5^{\circ}$ & $180^{\circ}$ & $202.5^{\circ}$ & $225^{\circ}$ & $247.5^{\circ}$ & $270^{\circ}$ & $292.5^{\circ}$ & $315^{\circ}$ \\
End $\phi$ & $190^{\circ}$ & $212.5^{\circ}$ & $235^{\circ}$ & $257.5^{\circ}$ & $280^{\circ}$ & $302.5^{\circ}$ & $325^{\circ}$ & $347.5^{\circ}$ \\
\hline
\end{tabular}

In $\eta$ the detector is divided into four intervals with overlap due to the finite size of the beam's luminous region in $z$ (Figure 2(b) 32). The boundary between barrel and end-cap towers is based on the edge of the most outer layer of the SCT barrels, where $\cos \theta \sim 35^{\circ}(-\sim$ $\left.35^{\circ}\right)$ and $\eta \sim 1.17(-1.17)$ with respect to the detector origin. Many modules located at Pixel barrel layers are assigned to the end-cap FTK towers. Since the boundary overlap is designed to cover collisions at $-120 \mathrm{~mm}<z<120 \mathrm{~mm}$, then the boundary is defined with respect to $z_{0}=-120 \mathrm{~mm}$ and $z_{0}=120 \mathrm{~mm}$. Modules satisfying $\eta<-1.3$ with respect to $z_{0}=120 \mathrm{~mm}$ (black line) are assigned to "C-Side Endcap", $\eta>-1.03$ with respect to $z_{0}=-120 \mathrm{~mm}$ and $\eta<-0.23$ with respect to $z_{0}=120 \mathrm{~mm}$ (red lines) are assigned to "C-Side Barrel" towers, $\eta>0.23$ with respect to $z_{0}=-120 \mathrm{~mm}$ and $\eta<1.03$ with respect to $z_{0}=120 \mathrm{~mm}$ (green lines) are assigned to "A-Side Barrel" towers, and $\eta>1.3$ with respect to $z_{0}=-120 \mathrm{~mm}$ (blue line) are assigned to "A-Side Endcap" towers. Significant numbers of modules are shared by neighboring towers in the inner layers.

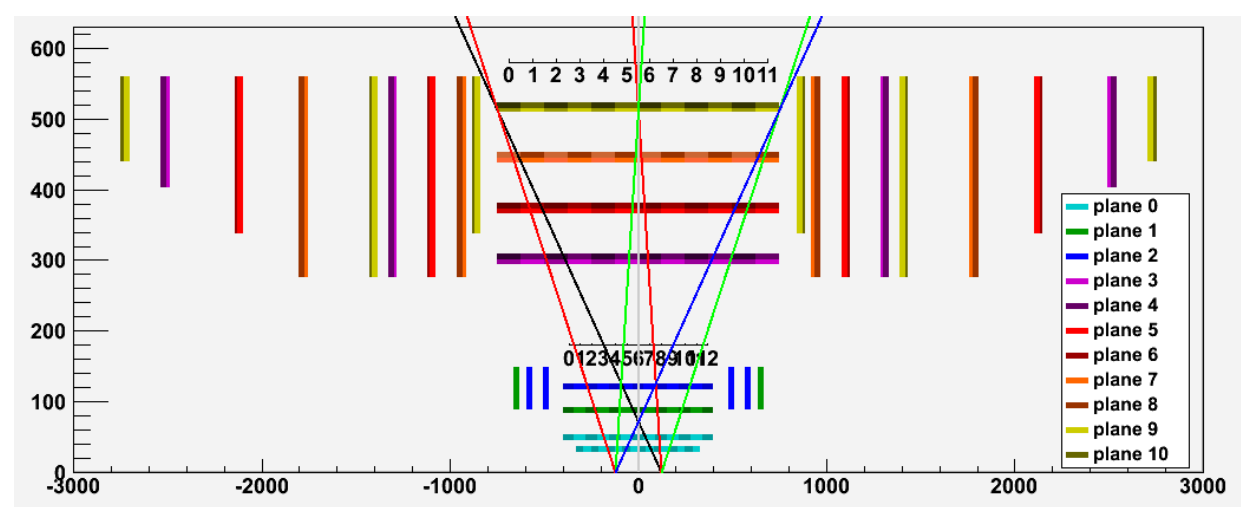

Figure 32: FTK Tower $\eta$ boundaries. 


\section{Appendix B AdvancedTCA Hardware Overview}

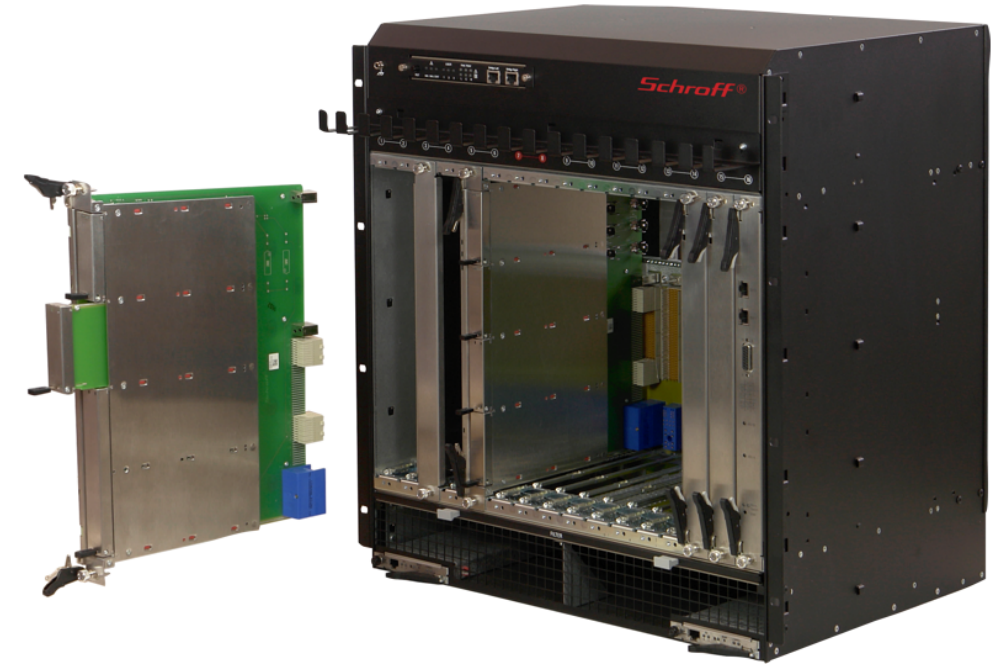

Figure 33: An ATCA board and 14-slot shelf.

Virtually every component in the ATCA shelf is a "field replaceable unit" (FRU) which means it may be replaced without powering down the shelf. Boards, fans, power entry modules, and shelf manager boards are hot-swappable and redundant. From the ground up ATCA has been designed for high availability operation.

\section{B.1 Shelf}

Boards are inserted into the ATCA shelf slots. Large shelf units contain 14 slots in a vertical configuration; smaller shelf units generally orient the blades horizontally. A typical 14 slot ATCA shelf is shown in Figure 33. For our application a 14 slot shelf will be used.

Each board is $8 \mathrm{U}(322.25 \mathrm{~mm})$ by $280 \mathrm{~mm}$ deep. The width of each slot is considerably wider than VME at $30 \mathrm{~mm}$, which allows for taller components such as connectors, mezzanine cards, power converters, and large capacitors.

\section{B.2 Backplane}

PICMG 3.0 specifies three backplane connector zones. Zone- 1 is near the bottom of the board and this connector is used for redundant 48VDC power and Intelligent Platform Management Controller (IPMC) management signals.

High speed data communication between boards occurs on the Zone-2 connectors. A few clocks and other synchronization signals are bused to all slots in the shelf, however a vast majority of the Zone-2 connections are point to point high speed serial links. ATCA is often described as "protocol agnostic" which means that the PICMG 3.0 specification simply describes the physical and electrical characteristics of these connections. The high speed serial data protocol is user defined. Zone-2 is comprised of two type of connections: the Base Interface and Fabric Interface.

The Base Interface is wired as dual star topology. There are two redundant hub slots in logical slots 1 and 2. Each hub slot has a direct connection to every other slot in the shelf. The Base Interface protocol is TCP/IP over Ethernet (100BASE-T or 1000BASE-T) and is intended for out of band management operations such as board control and monitoring. 


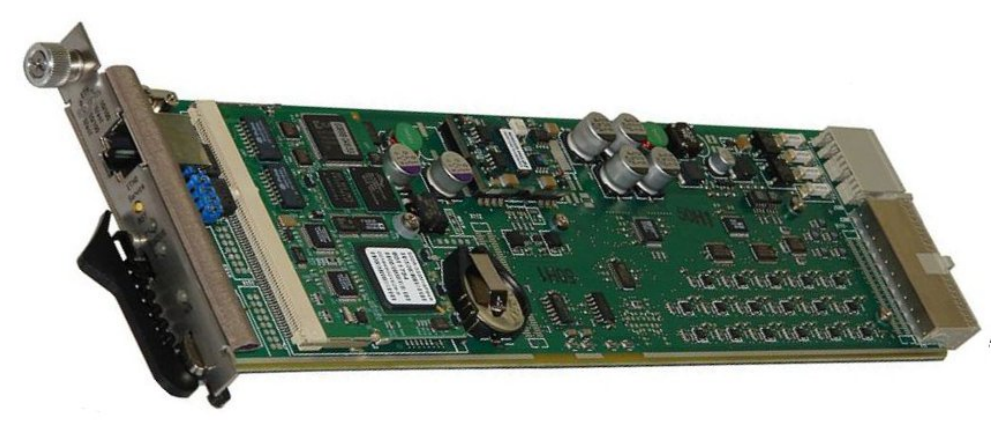

Figure 34: A Shelf Manager board.

High speed data transfers take place on the Zone-2 Fabric Interface. The Fabric Interface is available in full-mesh, dual-star, dual-dual-star and replicated-mesh topologies. The Data Formatter will use the full-mesh configuration, which features eight $100 \Omega$ differential signal pairs (4 RX pairs and 4 TX pairs) between each slot. Each differential signal pair is rated for speeds up to $10 \mathrm{~Gb} / \mathrm{s}$ (high-performance " $40 \mathrm{G}$ " backplanes) or up to $3 \mathrm{Gbps}$ ("standard" backplanes made with FR4 material).

The final backplane region is the Zone-3 area at top of the backplane. Connectors in the Zone-3 area are intended for passing data from the front board to the rear transition module (RTM). There is no backplane in this zone; rather the front board connectors mate directly with the connectors on the RTM. In the original PICMG 3.0 standard this zone was userdefined. The new PICMG 3.8 standard [6] defines the Zone-3 area as having three ADF style connectors for high speed serial data and a small blue ridiculously expensive connector for power and management.

\section{B.3 Backplane Clocks}

Data transfers across the Fabric Interface use high speed serial links, which embed the clock in the data stream (standard $8 \mathrm{~B} / 10 \mathrm{~B}$ encoding). Therefore, data transfers across the Fabric Interface are inherently self-clocking and do not require a separate master clock.

PICMG 3.0 does however define a set of clocks for general synchronization and timing. The Synchronization Clock Interface consists of six differential clock lines bused to all boards. ATCA boards may listen or drive any of these clock lines, as negotiated by the electronic keying feature of the IPMI management interface. Two of the four clocks (CLK3a and CLK3b) are user-defined and may be used for experiment synchronization and various DAQ control signals.

\section{B.4 Update Interface}

The Update Channel interface consists of 10 differential pairs in a point to point connection between two boards. This interface is optional and varies by backplane manufacturer. This interface is not used by the Data Formatter.

\section{B.5 Intelligent Platform Management Interface}

ATCA hardware incorporates an Intelligent Platform Management Interface (IPMI), which is required on all shelf components. Through this interface the Shelf Manager card can query sensors and control shelf components. For example, if the Shelf Manager detects an overtemperature condition on a board then the fan speed may be increased or the board could be powered down. 
High availability operation is archived through redundancy built into the IPMI specification. Each shelf has dual redundant Shelf Manager cards, one is shown in Figure 34. If the master Shelf Manager fails then control automatically transferred to the slave unit. Like other ATCA components the Shelf Manager boards support hot swap operation. The heart of the Shelf Manager card is a single board computer running Linux. It is possible to log into the Shelf Manager through telnet, SSH, or a serial terminal; however the user will typically interact with Shelf Manager through the web interface. An Ethernet port is located on the front panel of the Shelf Manager, and there are also jumpers to connect the Shelf Manager to the backplane Base Fabric network as well.

Shelf Manager cards communicate over the dual redundant Intelligent Platform Management Bus (IPMB), which uses the $\mathrm{I}^{2} \mathrm{C}$ protocol for the base layer. Typically the following sensors are monitored: temperature, fan speed, voltage and current, and board handle switch status. The board or FRU must also report back a description, serial number, manufacturer name, part number, and various hardware, firmware, and software version numbers. The IPMI protocols are fairly complex and a microcontroller (Intelligent Platform Management Controller, or IPMC) must be used.

Hot swap operation is implemented by monitoring the status of a microswitch in the board handle and controlling the DC-DC converters on the board. Removing a component from an ATCA shelf requires following a simple procedure which involves opening the ejector handle slightly, then watching the blue HS LED until it indicates that the board has completed the shutdown procedure, then the board may be removed safely from the system.

The IPMI specification supports firmware downloads to the IPMC microcontrollers. Downloading large FPGAs may be possible over the IPMI bus but this operation would be extremely slow given the $\mathrm{I}^{2} \mathrm{C}$ transmission speeds.

IPMC reference designs are available commercially available. The reference designs fully implement the latest IPMI specification and have been debugged and technical support is provided. However the commercial reference designs are strictly licensed, closed source, and discourage collaboration by requiring non-disclosure agreements. As an alternative, several HEP laboratories have produced open-source designs [8] for IPMC controllers.

\section{B.6 Network Connectivity}

All ATCA backplanes support a Dual Star Base Interface network which is based on Gigabit Ethernet. The Base Interface is generally used for high speed board management tasks such as board control and status and downloading firmware. Logical slots 1 and 2 form the hubs of two separate star Ethernet networks. Logical slots 3 through 14 each have two Base Interface ports: channel 1 connects to the slot 1 network and channel 2 connect to the slot 2 network. The Base Interface network uses 1000BASE-T Ethernet, which consists of 4 bidirectional signal pairs (similar to a "CAT5" cable). A small transformer and a PHY chip are required to interface to 1000BASE-T network.

The backplane Fabric Interface may also be used for high speed Ethernet communication between hubs and boards. The PICMG 3.1 specification details how 1000BASE-BX is used for Gigabit Ethernet (or 10G Ethernet) communication between node and hub boards. 1000BASE-BX consists of two differential pairs running at $1.25 \mathrm{Gbps}$ and is $8 \mathrm{~B} / 10 \mathrm{~B}$ encoded. 1000BASE-BX may be directly interfaced with an serial transceiver in an FPGA, no dedicated Ethernet PHY chip is required. The PICMG 3.1 specification also describes 10G Ethernet connections, which involve all four backplane ports in the channel "bonded" (synchronized) and running at $3.125 \mathrm{Gbps}$. 


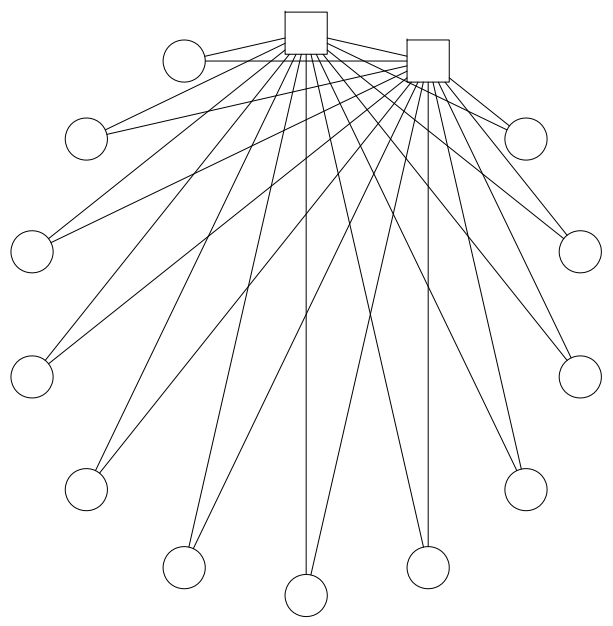

(a)

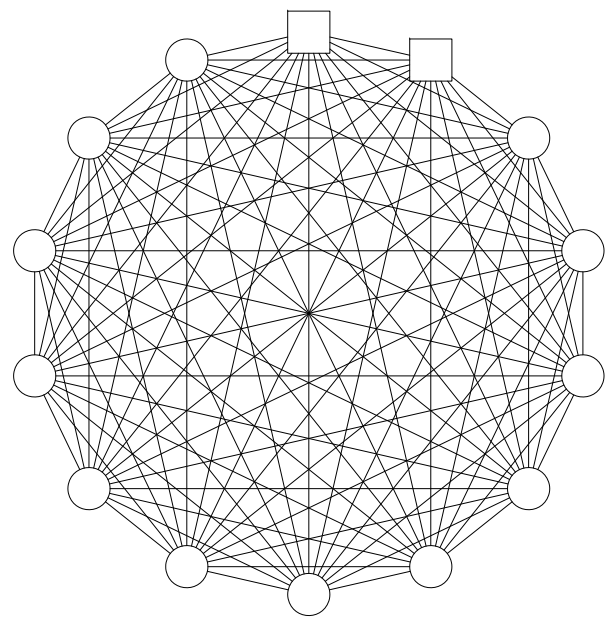

(b)

Figure 35: ATCA Backplane Connections. The Base Interface (a) consists of two independent 1000BASE-T Ethernet star networks. The Full Mesh Fabric Interface (b) connects all slots with bidirectional channels rated for up to 40Gbps. The Fabric Interface is "protocol agnostic".

\section{B.7 Hub and System Controller Boards}

ATCA System Controller boards combine a CPU, memory, hard drive and several Gigabit Ethernet network switches (Figure 36). Hub boards (or "switch blades") generally omit the CPU, memory and hard drive to reduce cost. All board types will at a minimum support a Gigabit Ethernet Base Interface switch. More advanced boards may provide higher speeds (10GbE), high performance multi-core CPUs, and support for a switch on the Fabric Interface.

\section{B.8 Power Supply}

ATCA evolved out of the telecommunications industry, which has historically used a -48VDC power distribution. The shelf incorporates redundant power entry modules, each of which has a connection for the $-48 \mathrm{~V}$ supply and return lines. ATCA hardware supports up to $200 \mathrm{~W}$ per slot.

The Power supplies are also redundant. A common configuration is a $1 \mathrm{U}$ rackmount chassis with three power supplies which operate in an "N+1" redundant mode. Output diodes and special circuitry is employed to implement dynamic load sharing and hot swap capability. Therefore, a failed supply can be shutdown or replaced without interrupting shelf operation.

Our experience with 48VDC "N+1" redundant power supplies has been extremely positive. For instance, when a power supply fails it is shut down and the other supplies automatically take up the load without interruption. Then, during a normally scheduled controlled access the faulty supply is simply replaced. Local voltage regulation on the board (with isolated DC-DC converters) is reliable and eliminates the need for remote sensing which is common on low-voltage high-current power supplies. Compared to a large low-voltage high-current power supply a board mounted DC-DC converter can simply react faster to the highly dynamic load often associated with high performance FPGAs, resulting in improved voltage regulation. 


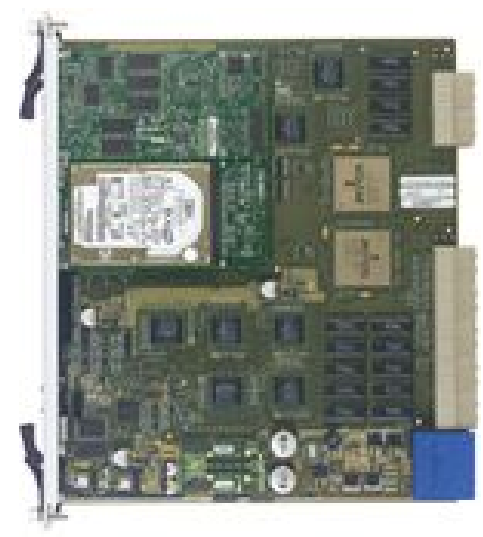

(a)

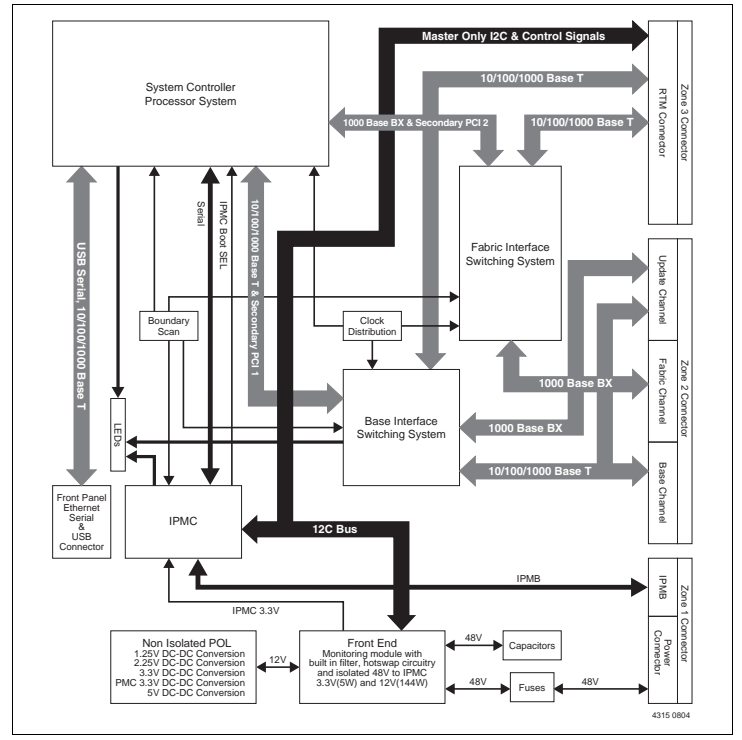

(b)

Figure 36: The Motorola ATCA F101 System Controller and "Switch Blade". This board combines a single-core CPU with a Gigabit Ethernet Base Interface switch (1000BASE-T). The CPU also connects to a Gigabit Ethernet Fabric Interface switch (1000BASE-BX, PICMG $3.1)$.

\section{Appendix C Mezzanine Card Details}

\section{C.1 FMC Pinout}

Notes:

1. LPC connectors populate rows C, D, G and H (160 pins).

2. HPC connectors populate all rows (400 pins).

3. The Data Formatter board supports all colored pins. All other pins are not connected.

4. The Data Formatter board sets VADJ to +3.3VDC.

5. PRSNT_M2C_L should be grounded on the mezzanine card.

6. SCL and SDA have 4.7k pullup resistors to $+3.3 \mathrm{~V}$ on the Data Formatter board.

7. Refer to the Samtec website [15] for HPC and LPC connector datasheets and drawings. 


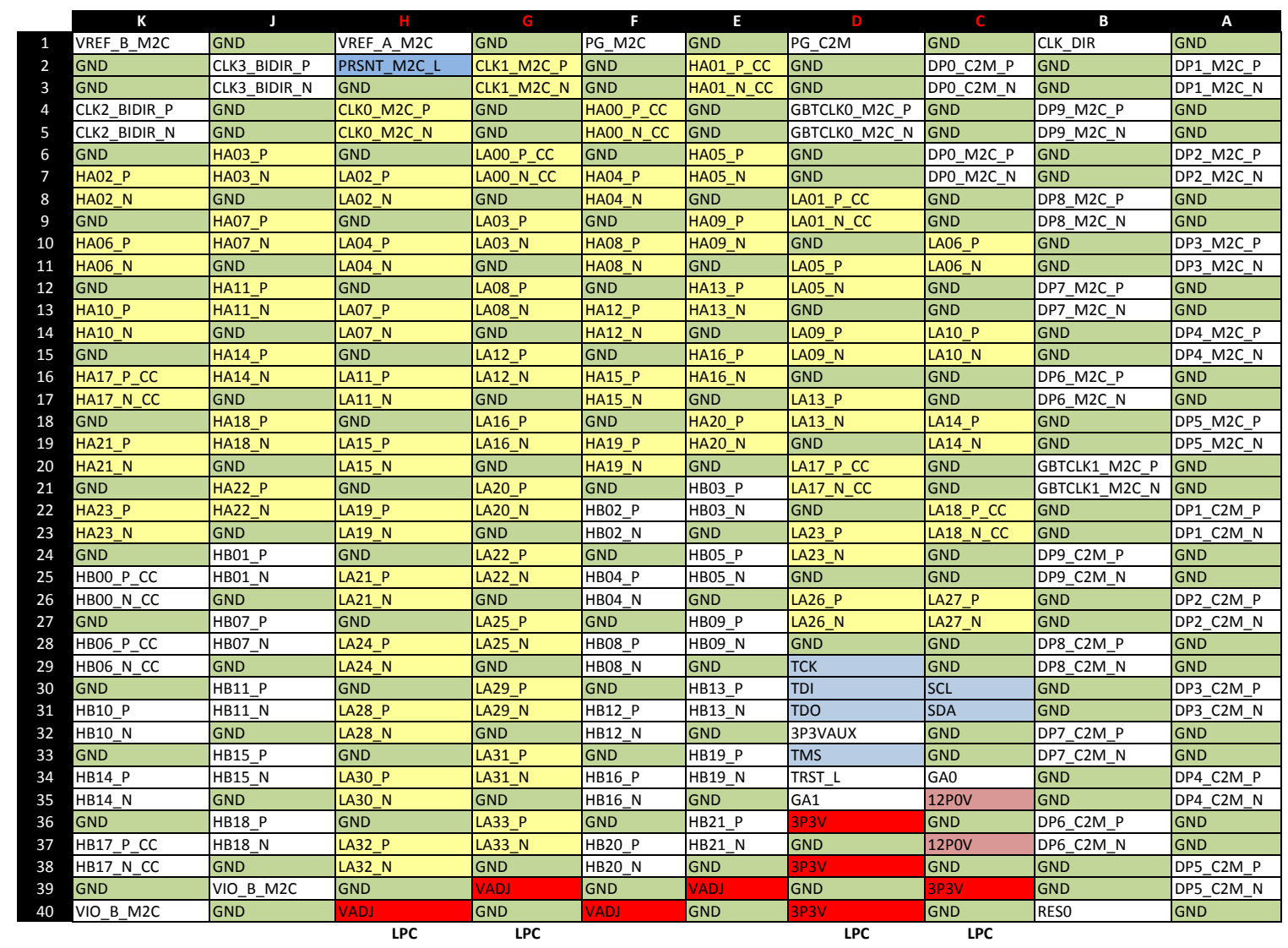

Table 15: The FMC Connector Pinout. The Data Formatter board supports a subset of High Pin Count (HPC) connections. 


\section{C.2 FMC Connector}

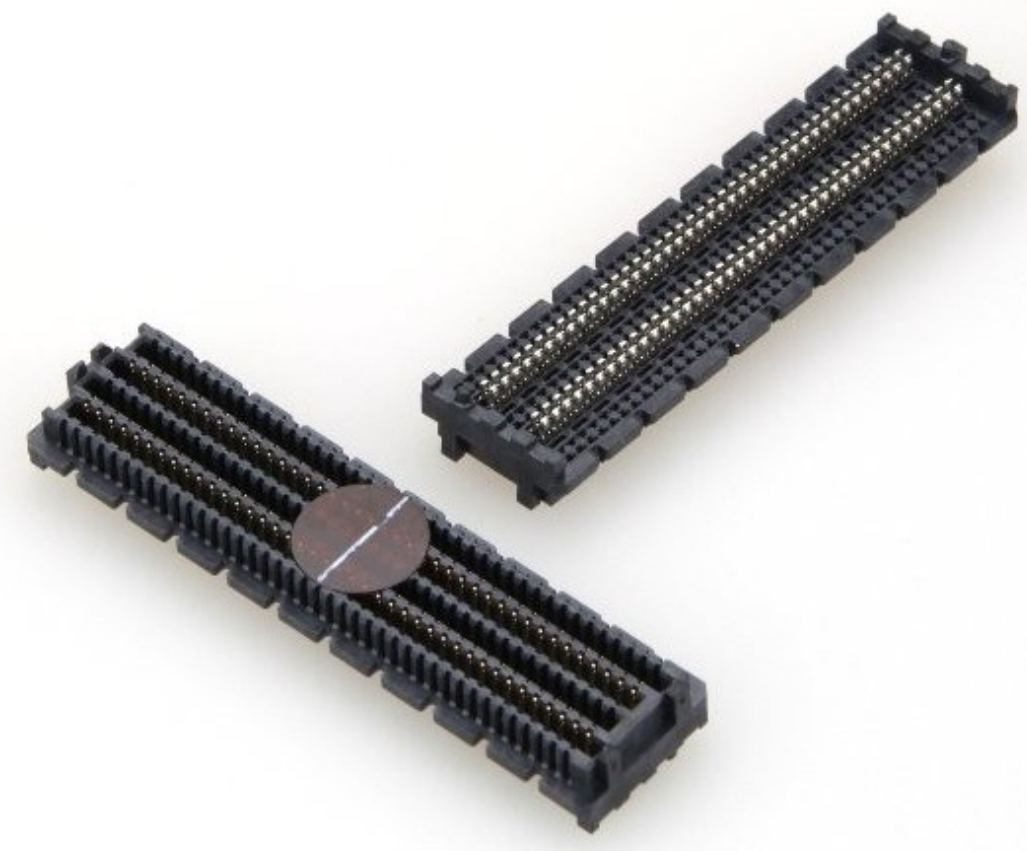

Figure 37: The male FMC connector, which is soldered on the mezzanine card. The male connector is available in $8.5 \mathrm{~mm}$ and $10 \mathrm{~mm}$ stack height versions. The LPC version is shown here. 


\section{C.3 Dimensions}

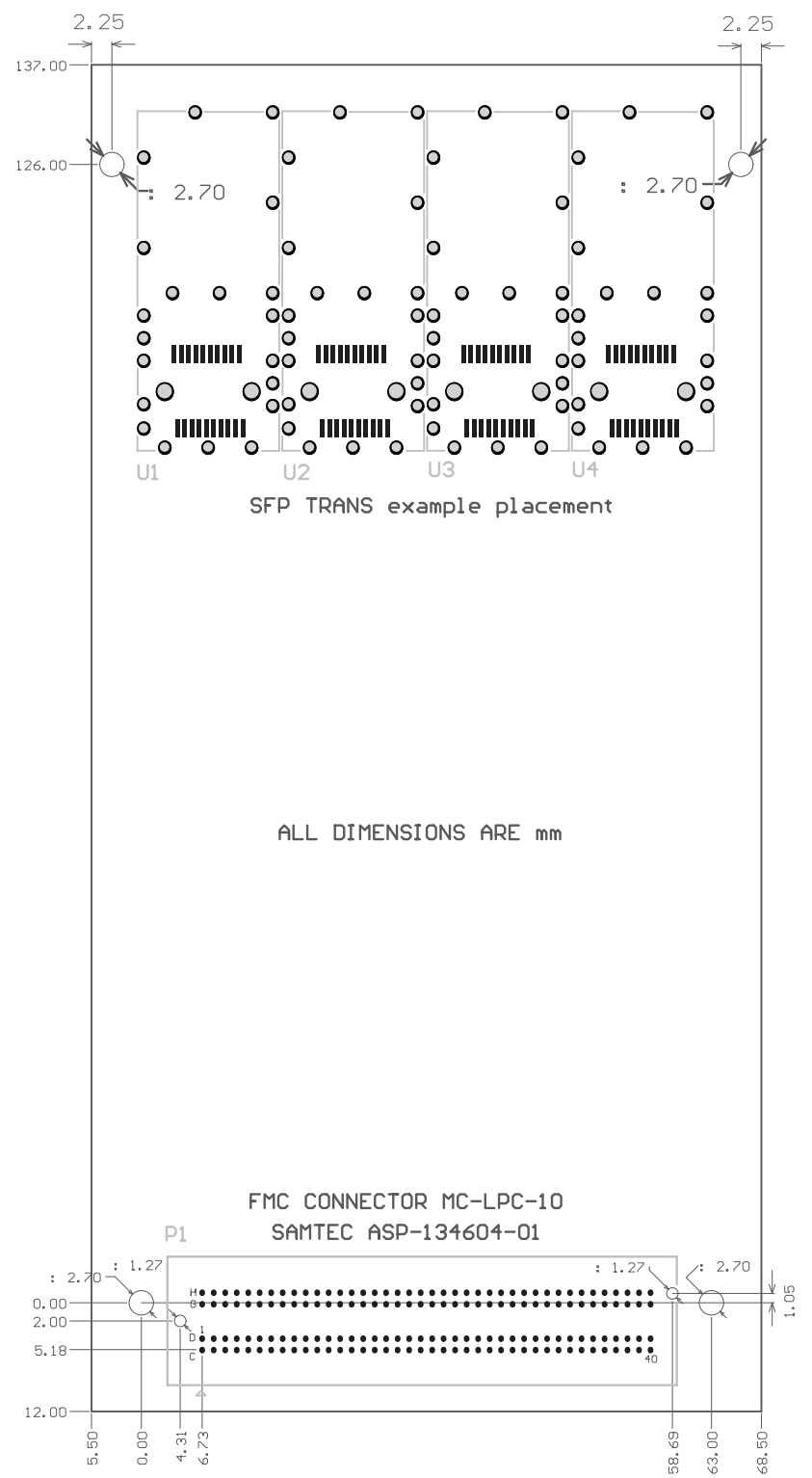

Figure 38: The mezzanine card as viewed looking at the "component side" of the board, as in Figure 13. A low pin count (LPC) connector is shown here. 


\section{C.4 Bezel Detail}

Mezzanine cards use the standard CMC/PMC bezel, which measures $10 \mathrm{~mm}$ high by $74 \mathrm{~mm}$ wide. When installed on a Data Formatter board the bezel will be flush with the front panel. Although space is tight, up to four SFP optical transceivers may be mounted on a mezzanine card.

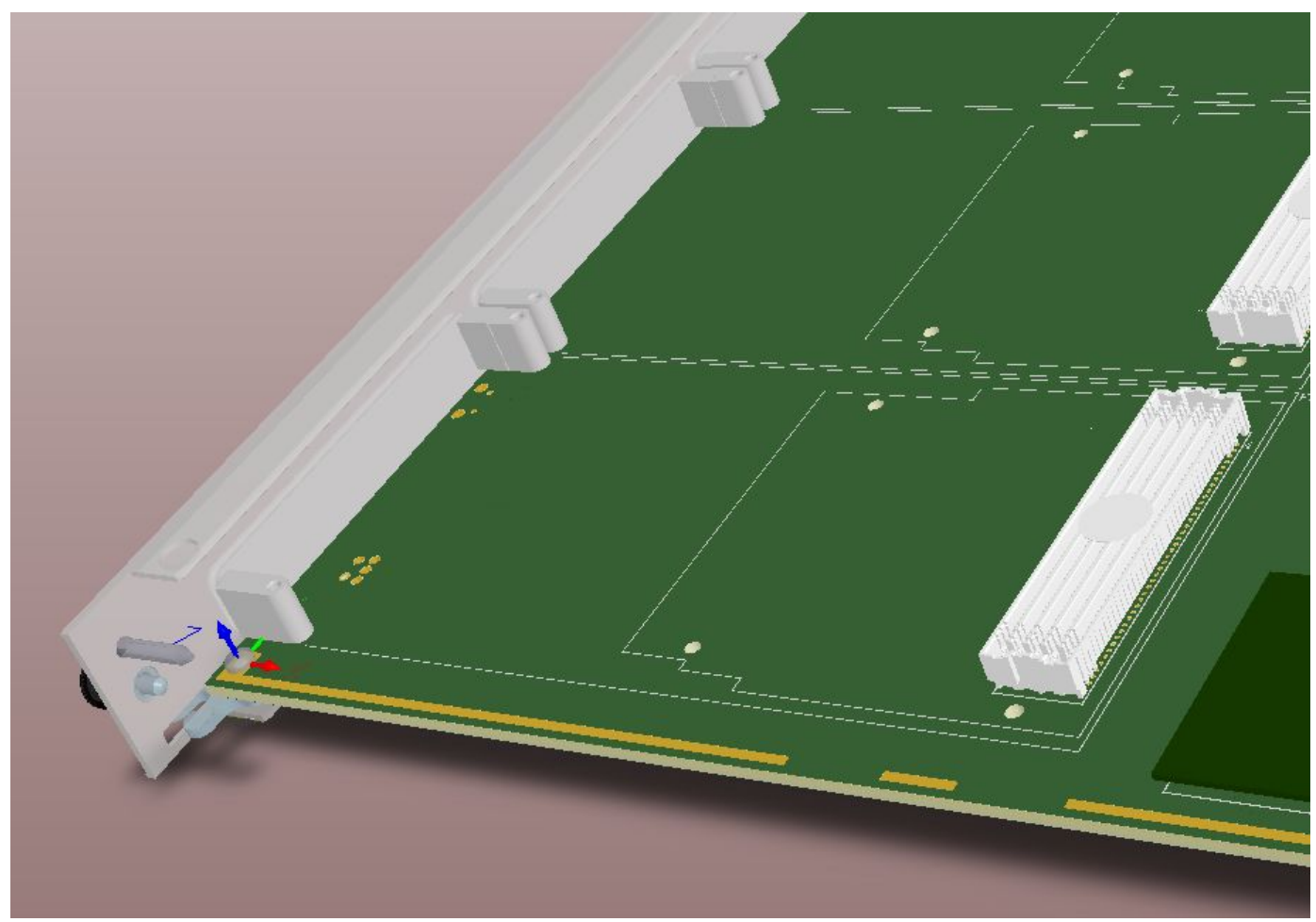

Figure 39: The Data Formatter board detail showing the mezzanine card FMC connector, bezel and front panel. 


\section{Appendix D RTM Details}

D.1 Transceiver Numbering

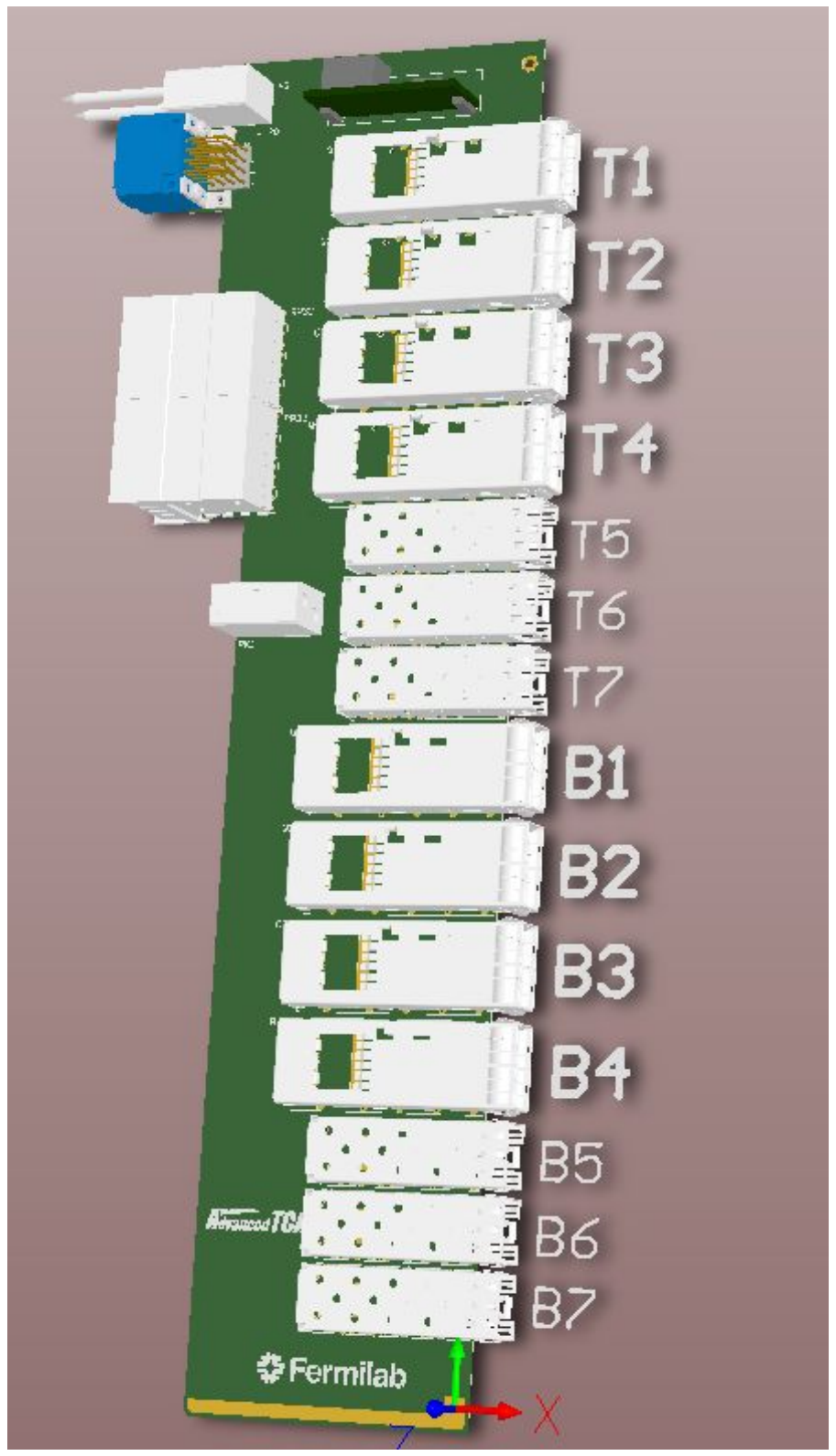

Figure 40: RTM transceiver identifiers 


\section{D.2 Transceiver Connector Port Assignments}

\begin{tabular}{|l|l|l|l|}
\hline Connector & Port Number & RTM Transceiver & Transceiver Channel \\
\hline \hline RP32 & 19 & QSFP T1 & 1 \\
\hline RP32 & 18 & QSFP T1 & 2 \\
\hline RP32 & 17 & QSFP T1 & 3 \\
\hline RP32 & 16 & QSFP T1 & 4 \\
\hline RP32 & 15 & QSFP T2 & 1 \\
\hline RP32 & 14 & QSFP T2 & 2 \\
\hline RP32 & 13 & QSFP T2 & 3 \\
\hline RP32 & 12 & QSFP T2 & 4 \\
\hline RP32 & 11 & QSFP T3 & 1 \\
\hline RP32 & 10 & QSFP T3 & 2 \\
\hline RP32 & 9 & QSFP T3 & 3 \\
\hline RP32 & 8 & QSFP T3 & 4 \\
\hline RP32 & 7 & QSFP T4 & 1 \\
\hline RP32 & 6 & QSFP T4 & 2 \\
\hline RP32 & 5 & QSFP T4 & 3 \\
\hline RP32 & 4 & QSFP T4 & 4 \\
\hline RP32 & 3 & SFP T5 & n/a \\
\hline RP32 & 2 & SFP T6 & n/a \\
\hline RP32 & 1 & SFP T7 & n/a \\
\hline RP32 & 0 & (reserved) & n/a \\
\hline & & & \\
\hline
\end{tabular}

Table 16: Port Assignments for Zone-3A connectors J32/RP32. These transceivers connect to the top FPGA. 


\begin{tabular}{|l|l|l|l|}
\hline Connector & Port Number & RTM Transceiver & Transceiver Channel \\
\hline \hline RP33 & 19 & QSFP B1 & 1 \\
\hline RP33 & 18 & QSFP B1 & 2 \\
\hline RP33 & 17 & QSFP B1 & 3 \\
\hline RP33 & 16 & QSFP B1 & 4 \\
\hline RP33 & 15 & QSFP B2 & 1 \\
\hline RP33 & 14 & QSFP B2 & 2 \\
\hline RP33 & 13 & QSFP B2 & 3 \\
\hline RP33 & 12 & QSFP B2 & 4 \\
\hline RP33 & 11 & QSFP B3 & 1 \\
\hline RP33 & 10 & QSFP B3 & 2 \\
\hline RP33 & 9 & QSFP B3 & 3 \\
\hline RP33 & 8 & QSFP B3 & 4 \\
\hline RP33 & 7 & QSFP B4 & 1 \\
\hline RP33 & 6 & QSFP B4 & 2 \\
\hline RP33 & 5 & QSFP B4 & 3 \\
\hline RP33 & 4 & QSFP B4 & 4 \\
\hline RP33 & 3 & SFP B5 & n/a \\
\hline RP33 & 2 & SFP B6 & n/a \\
\hline RP33 & 1 & SFP B7 & n/a \\
\hline RP33 & 0 & (reserved) & n/a \\
\hline & & & \\
\hline
\end{tabular}

Table 17: Port Assignments for Zone-3A connectors J33/RP33. These transceivers connect to the bottom FPGA. 


\section{D.3 Rear Panel Detail}

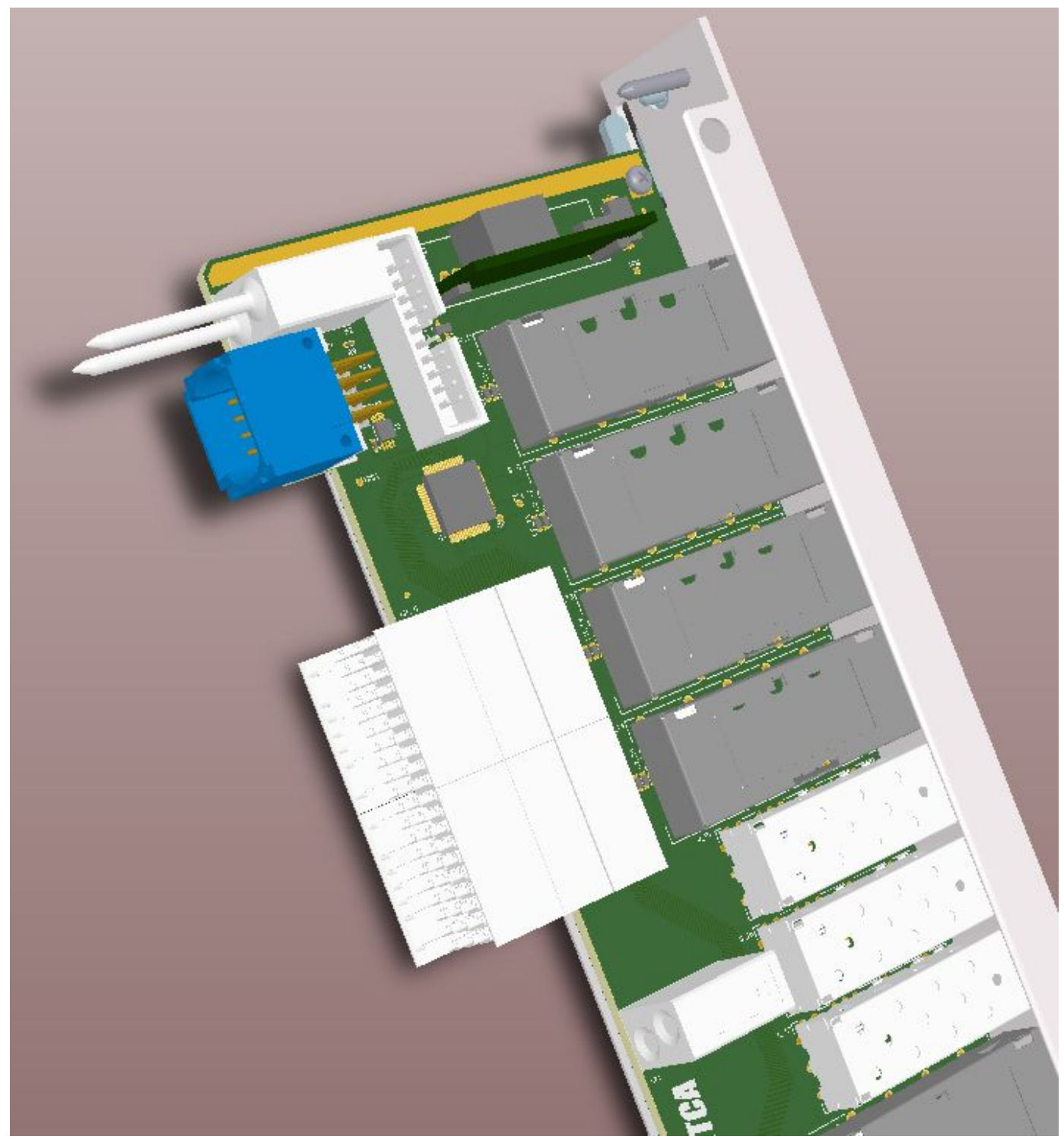

Figure 41: RTM detail showing the PICMG 3.8 management connector (blue), the high speed ADF connectors RP32 and RP33. A small ARM microcontroller and debug/programming header is shown. RTM transceivers are powered by the $3.3 \mathrm{~V} 20 \mathrm{~A}$ switching regulator shown at the top of the card. 


\section{Appendix E Initial Firmware Study}

Firmware development is ongoing and we have the Xilinx Kintex development board [10] on hand. In the following sections we discuss general firmware development, FPGA performance tests, and present some initial thoughts about a packet switch algorithm that permits high speed switching the in Data Formatter FPGAs.

\section{E.1 Serial Transceivers}

Xilinx Kintex FPGAs use internal multi-gigabit serial transceivers, commonly referred to as MGTs or GTX modules. In the highest speed grade devices the GTX transceivers support data rates up to $12.5 \mathrm{Gbps}$.

The SLINK firmware was originally developed for FPGAs with external SERDES chips. Subsequent incarnations used internal SERDES components but have targeted ALTERA FPGAs primitives. We have successfully adapted the SLINK firmware to use Kintex GTX transceivers.

Serial links internal to the Data Formatter system will likely use the Aurora protocol. Aurora is a light-weight low-overhead protocol which supports basic flow control and data integrity checking. We are currently testing the Aurora-GTX firmware core designs.

FPGA I/O pin resources have evolved rapidly over the past few years. In the Kintex family each I/O pin contains a serializer (OSERDES2) and de-serializer (ISERDES2). These components allow for serial communications up to 1.6Gbps per LVDS pin pair without using any GTX resources. We intend to use these I/O serializer functions for high speed communication between the Data Formatter FPGAs and the Mezzanine Cards.

\section{E.2 Packet Switch Preliminaty Consideration}

There is no central switch or router in the Data Formatter system. Data packets are routed between FPGAs in a distributed routing scheme. Although the connections between FPGAs are numerous (see Figure 6) not all FPGAs are directly connected with point to point links. Therefore it may be necessary for the FPGA routing logic to support "route through" or packet re-transmission. For example, a packet entering the FPGA on Fabric Interface channel 3 may be re-transmitted on the local-bus, or it may go to another shelf over the inter-shelf link, or even another Fabric Interface channel.

As a data packet arrives in the FPGA (from any input) its module-ID is evaluated against a routing table and the destination FPGAs output (or outputs) is calculated. The packet is then sent through a possible switch network implemented in the FPGA logic, as shown in Figure 42. A few switch input ports are reserved for the mezzanine cards and a few switch output ports are reserved for the formatting logic, which concatenates and repackages the data before transmission to the AUX cards and SSB boards.

A simple "crossbar" network switch architecture is inherently blocking and would cause latency to necessarily increase. An alternative switch architecture based on a banyan network is shown in Figure 43. The banyan network consists of switch nodes which include local buffering in FIFOs. We are currently simulating the performance of such a switch and evaluating the FPGA logic resources required.

Our data volume analysis (see Section 8) shows that not all links are equally utilized. Under-utilized links may be used more effectively by tweaking the routing tables in each FPGA. In some cases, an alternate or less direct route involving lightly-used links may be more advantageous in terms of latency and overall throughput. A switch architecture that supports "route through" allows for link-utilization optimization. We are currently evaluating the feasibility of such optimization. 


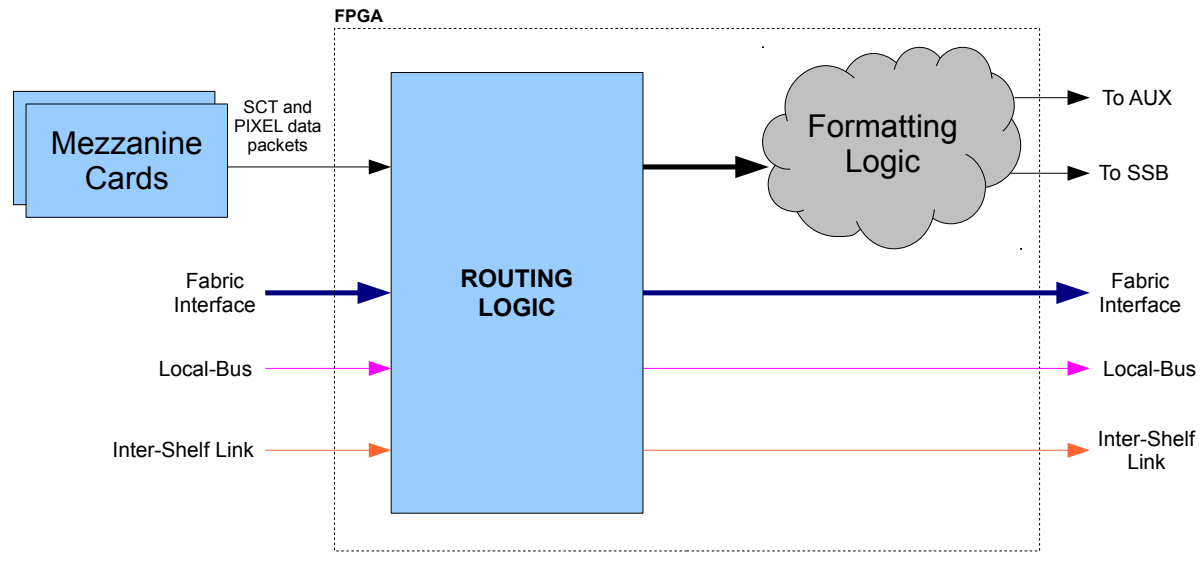

Figure 42: Overview of the FPGA routing firmware. Data packets containing SCT and Pixel hits and clusters are output from a pair of mezzanine cards. Data packets also arrive on the Fabric Interface channels, the local-bus link, and inter-shelf link. It is possible for the firmware to support "route through" which enables an incoming packet to be re-transmitted over any output.
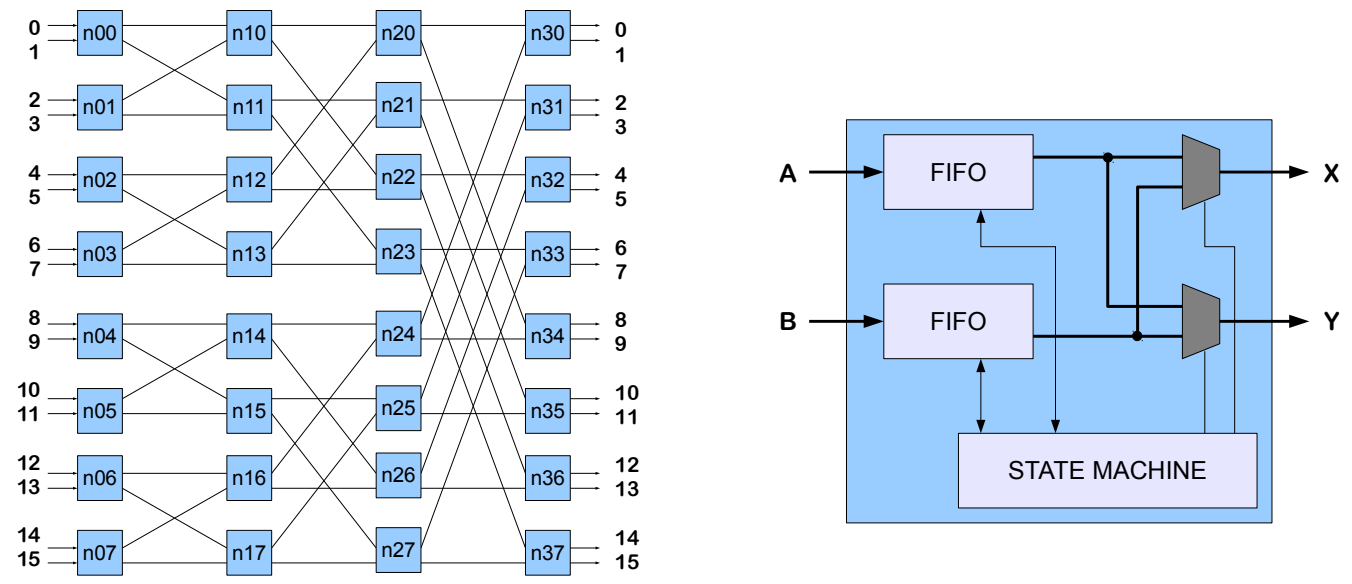

Figure 43: A possible 16x16 port network switch implemented in the FPGA logic. The switch nodes (right) contains FIFOs for internal data buffering. 


\section{Appendix F ROD IDs}

ROD ID numbers used in Figure 28, Figure 29, Figure 30 and Figure 31 are summarized for 222 RODs in the current existing ATLAS DAQ system $[17,18]$.

\section{F.1 132 Pixel RODs}

\begin{tabular}{|c|c|c|c|}
\hline ID & ROD Address & Side & Barrel/Endcap \\
\hline 1 & $0 \times 00130005$ & $\mathrm{C}$ & Barrel \\
\hline 2 & $0 \mathrm{x} 00130006$ & $\mathrm{~A}$ & Barrel \\
\hline 3 & $0 \times 00130007$ & $\mathrm{~A}$ & Barrel \\
\hline 4 & 0x00130008 & $\mathrm{C}$ & Barrel \\
\hline 5 & $0 \times 00130009$ & $\mathrm{C}$ & Barrel \\
\hline 6 & $0 \mathrm{x} 00130010$ & $\mathrm{~A}$ & Barrel \\
\hline 7 & 0x00130011 & A & Barrel \\
\hline 8 & $0 \times 00130012$ & $\mathrm{C}$ & Barrel \\
\hline 9 & $0 \times 00130014$ & $\mathrm{C}$ & Barrel \\
\hline 10 & $0 \times 00130015$ & $\mathrm{~A}$ & Barrel \\
\hline 11 & $0 x 00130016$ & $\mathrm{~A}$ & Barrel \\
\hline 12 & $0 \times 00130017$ & $\mathrm{C}$ & Barrel \\
\hline 13 & $0 \mathrm{x} 00130018$ & $\mathrm{C}$ & Barrel \\
\hline 14 & $0 \times 00130019$ & $\mathrm{~A}$ & Barrel \\
\hline 15 & $0 \mathrm{x} 00130020$ & $\mathrm{~A}$ & Barrel \\
\hline 16 & $0 \times 00130021$ & $\mathrm{C}$ & Barrel \\
\hline 17 & $0 \times 00130105$ & $\mathrm{C}$ & Barrel \\
\hline 18 & $0 \times 00130106$ & $\mathrm{~A}$ & Barrel \\
\hline 19 & $0 \times 00130107$ & $\mathrm{~A}$ & Barrel \\
\hline 20 & $0 \times 00130108$ & $\mathrm{C}$ & Barrel \\
\hline 21 & 0x00130109 & $\mathrm{C}$ & Barrel \\
\hline 22 & $0 \times 00130110$ & $\bar{A}$ & Barrel \\
\hline 23 & $0 \times 00130111$ & $\mathrm{~A}$ & Barrel \\
\hline 24 & $0 x 00130112$ & $\mathrm{C}$ & Barrel \\
\hline 25 & $0 \times 00130114$ & $\mathrm{C}$ & Barrel \\
\hline 26 & $0 \times 00130115$ & $\mathrm{~A}$ & Barrel \\
\hline 27 & $0 \mathrm{x} 00130116$ & $\mathrm{~A}$ & Barrel \\
\hline 28 & $0 \times 00130117$ & $\mathrm{C}$ & Barrel \\
\hline 29 & 0x00130118 & $\mathrm{C}$ & Barrel \\
\hline 30 & $0 \times 00130119$ & $\bar{A}$ & Barrel \\
\hline 31 & $0 \times 00130120$ & $\mathrm{~A}$ & Barrel \\
\hline 32 & $0 \times 00130121$ & $\mathrm{C}$ & Barrel \\
\hline 33 & $0 x 00130307$ & $\mathrm{C}$ & Barrel \\
\hline 34 & $0 \times 00130308$ & $\mathrm{~A}$ & Barrel \\
\hline 35 & 0x00130309 & $\mathrm{A}$ & Barrel \\
\hline 36 & $0 \times 00130310$ & $\mathrm{C}$ & Barrel \\
\hline 37 & $0 \mathrm{x} 00130311$ & $\mathrm{C}$ & Barrel \\
\hline 38 & $0 \mathrm{x} 00130312$ & $\mathrm{~A}$ & Barrel \\
\hline 39 & $0 \mathrm{x} 00130314$ & $\mathrm{~A}$ & Barrel \\
\hline 40 & $0 \times 00130315$ & $\mathrm{C}$ & Barrel \\
\hline 41 & $0 x 00130316$ & $\mathrm{C}$ & Barrel \\
\hline 42 & $0 \times 00130317$ & $\mathrm{~A}$ & Barrel \\
\hline 43 & $0 x 00130318$ & $\mathrm{~A}$ & Barrel \\
\hline 44 & 0x00130319 & $\mathrm{C}$ & Barrel \\
\hline
\end{tabular}

\begin{tabular}{|c|c|c|c|}
\hline ID & ROD Address & Side & Barrel/Endcap \\
\hline 45 & 0x00120205 & C & Endcap \\
\hline 46 & 0x00120206 & A & Endcap \\
\hline 47 & 0x00120207 & C & Endcap \\
\hline 48 & 0x00120208 & A & Endcap \\
\hline 49 & 0x00120209 & C & Endcap \\
\hline 50 & 0x00120210 & A & Endcap \\
\hline 51 & 0x00120211 & C & Endcap \\
\hline 52 & 0x00120212 & A & Endcap \\
\hline 53 & 0x00120214 & C & Endcap \\
\hline 54 & 0x00120215 & A & Endcap \\
\hline 55 & 0x00120216 & C & Endcap \\
\hline 56 & 0x00120217 & A & Endcap \\
\hline 57 & 0x00120218 & C & Endcap \\
\hline 58 & 0x00120219 & A & Endcap \\
\hline 59 & 0x00120220 & C & Endcap \\
\hline 60 & 0x00120221 & A & Endcap \\
\hline 61 & 0x00121609 & C & Endcap \\
\hline 62 & 0x00121610 & A & Endcap \\
\hline 63 & 0x00121611 & C & Endcap \\
\hline 64 & 0x00121612 & A & Endcap \\
\hline 65 & 0x00121614 & C & Endcap \\
\hline 66 & 0x00121615 & A & Endcap \\
\hline 67 & 0x00121616 & C & Endcap \\
\hline 68 & 0x00121617 & A & Endcap \\
\hline
\end{tabular}




\begin{tabular}{|c|c|c|c|}
\hline ID & ROD Address & Side & Barrel/Endcap \\
\hline 69 & $0 \times 00112405$ & $\mathrm{~A}$ & Barrel \\
\hline 70 & $0 \mathrm{x} 00112406$ & $\mathrm{C}$ & Barrel \\
\hline 71 & 0x00112407 & $\mathrm{A}$ & Barrel \\
\hline 72 & $0 \times 00112408$ & $\mathrm{C}$ & Barrel \\
\hline 73 & $0 \times 00112409$ & $\mathrm{~A}$ & Barrel \\
\hline 74 & $0 \times 00112410$ & $\mathrm{C}$ & Barrel \\
\hline 75 & $0 x 00112411$ & $\mathrm{~A}$ & Barrel \\
\hline 76 & $0 x 00112412$ & $\mathrm{C}$ & Barrel \\
\hline 77 & $0 \times 00112414$ & $\mathrm{~A}$ & Barrel \\
\hline 78 & $0 \times 00112415$ & $\mathrm{C}$ & Barrel \\
\hline 79 & $0 \times 00112416$ & $\mathrm{~A}$ & Barrel \\
\hline 80 & $0 \times 00112417$ & $\mathrm{C}$ & Barrel \\
\hline 81 & $0 \times 00112418$ & $\mathrm{~A}$ & Barrel \\
\hline 82 & $0 \times 00112419$ & $\mathrm{C}$ & Barrel \\
\hline 83 & $0 \times 00112420$ & $\mathrm{~A}$ & Barrel \\
\hline 84 & $0 \times 00112421$ & $\mathrm{C}$ & Barrel \\
\hline 85 & $0 \times 00112505$ & $\mathrm{C}$ & Barrel \\
\hline 86 & $0 \times 00112506$ & $\mathrm{~A}$ & Barrel \\
\hline 87 & $0 \times 00112507$ & $\mathrm{C}$ & Barrel \\
\hline 88 & $0 \times 00112508$ & $\mathrm{~A}$ & Barrel \\
\hline 89 & $0 \times 00112509$ & $\mathrm{C}$ & Barrel \\
\hline 90 & $0 \times 00112510$ & $\mathrm{~A}$ & Barrel \\
\hline 91 & $0 \times 00112511$ & $\mathrm{~A}$ & Barrel \\
\hline 92 & $0 \times 00112512$ & $\mathrm{C}$ & Barrel \\
\hline 93 & $0 \times 00112514$ & $\mathrm{~A}$ & Barrel \\
\hline 94 & $0 x 00112515$ & $\mathrm{C}$ & Barrel \\
\hline 95 & $0 x 00112516$ & $\mathrm{~A}$ & Barrel \\
\hline 96 & $0 \times 00112517$ & $\mathrm{C}$ & Barrel \\
\hline 97 & $0 \times 00112518$ & $\mathrm{~A}$ & Barrel \\
\hline 98 & $0 \times 00112519$ & $\mathrm{C}$ & Barrel \\
\hline 99 & $0 x 00112520$ & $\mathrm{~A}$ & Barrel \\
\hline 100 & $0 \times 00112521$ & $\mathrm{C}$ & Barrel \\
\hline
\end{tabular}

\begin{tabular}{|c|c|c|c|}
\hline ID & ROD Address & Side & Barrel/Endcap \\
\hline 101 & 0x00111705 & A & Barrel \\
\hline 102 & 0x00111706 & C & Barrel \\
\hline 103 & 0x00111707 & A & Barrel \\
\hline 104 & 0x00111708 & C & Barrel \\
\hline 105 & 0x00111709 & A & Barrel \\
\hline 106 & 0x00111710 & C & Barrel \\
\hline 107 & 0x00111711 & A & Barrel \\
\hline 108 & 0x00111712 & C & Barrel \\
\hline 109 & 0x00111714 & A & Barrel \\
\hline 110 & 0x00111715 & C & Barrel \\
\hline 111 & 0x00111716 & A & Barrel \\
\hline 112 & 0x00111717 & C & Barrel \\
\hline 113 & 0x00111718 & A & Barrel \\
\hline 114 & 0x00111719 & C & Barrel \\
\hline 115 & 0x00111720 & A & Barrel \\
\hline 116 & 0x00111721 & C & Barrel \\
\hline 117 & 0x00111805 & A & Barrel \\
\hline 118 & 0x00111806 & C & Barrel \\
\hline 119 & 0x00111807 & A & Barrel \\
\hline 120 & 0x00111808 & C & Barrel \\
\hline 121 & 0x00111809 & A & Barrel \\
\hline 122 & 0x00111810 & C & Barrel \\
\hline 123 & 0x00111811 & A & Barrel \\
\hline 124 & 0x00111812 & C & Barrel \\
\hline 125 & 0x00111814 & A & Barrel \\
\hline 126 & 0x00111815 & C & Barrel \\
\hline 127 & 0x00111816 & A & Barrel \\
\hline 128 & 0x00111817 & C & Barrel \\
\hline 129 & 0x00111818 & A & Barrel \\
\hline 130 & 0x00111819 & C & Barrel \\
\hline 131 & 0x00111820 & A & Barrel \\
\hline 132 & 0x00111821 & C & Barrel \\
\hline & & & \\
\hline
\end{tabular}




\section{F.2 90 SCT RODs}

\begin{tabular}{|c|c|c|c|}
\hline ID & ROD Address & Side & Barrel/Endcap \\
\hline 1 & 0x00220005 & C & Barrel \\
\hline 2 & 0x00210005 & A & Barrel \\
\hline 3 & 0x00220007 & C & Barrel \\
\hline 4 & 0x00210007 & A & Barrel \\
\hline 5 & 0x00220008 & C & Barrel \\
\hline 6 & 0x00210008 & A & Barrel \\
\hline 7 & 0x0022000a & C & Barrel \\
\hline 8 & 0x0021000a & A & Barrel \\
\hline 9 & 0x00220100 & C & Barrel \\
\hline 10 & 0x00210100 & A & Barrel \\
\hline 11 & 0x00220101 & C & Barrel \\
\hline 12 & 0x00210101 & A & Barrel \\
\hline 13 & 0x00220103 & C & Barrel \\
\hline 14 & 0x00210103 & A & Barrel \\
\hline 15 & 0x00220104 & C & Barrel \\
\hline 16 & 0x00210104 & A & Barrel \\
\hline 17 & 0x00220105 & C & Barrel \\
\hline 18 & 0x00210105 & A & Barrel \\
\hline 19 & 0x00220107 & C & Barrel \\
\hline 20 & 0x00210107 & A & Barrel \\
\hline 21 & 0x00220108 & C & Barrel \\
\hline 22 & 0x00210108 & A & Barrel \\
\hline 23 & 0x0022010a & C & Barrel \\
\hline 24 & 0x0021010a & A & Barrel \\
\hline 25 & 0x00220000 & C & Barrel \\
\hline 26 & 0x00210000 & A & Barrel \\
\hline 27 & 0x00220001 & C & Barrel \\
\hline 28 & 0x00210001 & A & Barrel \\
\hline 29 & 0x00220003 & C & Barrel \\
\hline 30 & 0x00210003 & A & Barrel \\
\hline 31 & 0x00220004 & C & Barrel \\
\hline 32 & 0x00210004 & A & Barrel \\
\hline 33 & 0x00220009 & C & Barrel \\
\hline 34 & 0x00210009 & A & Barrel \\
\hline 35 & 0x00220102 & C & Barrel \\
\hline 36 & 0x00210102 & A & Barrel \\
\hline 37 & 0x00220109 & C & Barrel \\
\hline 38 & 0x00210109 & A & Barrel \\
\hline 39 & 0x00220002 & C & Barrel \\
\hline 41 & 0x00210002 & A & Barrel \\
\hline 44 & 0x00220006 & C & Barrel \\
\hline
\end{tabular}

\begin{tabular}{|c|c|c|c|}
\hline ID & ROD Address & Side & Barrel/Endcap \\
\hline 45 & $0 \mathrm{x} 00230000$ & $\mathrm{~A}$ & Endcap \\
\hline 46 & 0x00230100 & $\mathrm{A}$ & Endcap \\
\hline 47 & 0x0023010b & A & Endcap \\
\hline 48 & 0x0023000a & A & Endcap \\
\hline 49 & 0x00230001 & $\mathrm{A}$ & Endcap \\
\hline 50 & $0 \mathrm{x} 00230002$ & $\bar{A}$ & Endcap \\
\hline 51 & 0x00230101 & $\mathrm{A}$ & Endcap \\
\hline 52 & 0x00230102 & A & Endcap \\
\hline 53 & 0x0023010a & A & Endcap \\
\hline 54 & 0x00230109 & $\bar{A}$ & Endcap \\
\hline 55 & $0 \times 00230009$ & A & Endcap \\
\hline 56 & $0 x 00230008$ & $\mathrm{~A}$ & Endcap \\
\hline 57 & 0x00230003 & A & Endcap \\
\hline 58 & 0x00230103 & $\bar{A}$ & Endcap \\
\hline 59 & 0x00230108 & A & Endcap \\
\hline 60 & $0 \mathrm{x} 00230007$ & $\mathrm{~A}$ & Endcap \\
\hline 61 & 0x00230004 & $\mathrm{A}$ & Endcap \\
\hline 62 & 0x00230107 & $\bar{A}$ & Endcap \\
\hline 63 & 0x00230104 & A & Endcap \\
\hline 64 & $0 \mathrm{x} 00230006$ & $\mathrm{~A}$ & Endcap \\
\hline 65 & 0x00230105 & $\mathrm{A}$ & Endcap \\
\hline 66 & 0x00230005 & $\mathrm{A}$ & Endcap \\
\hline 67 & 0x00230106 & A & Endcap \\
\hline 68 & 0x00240100 & $\mathrm{C}$ & Endcap \\
\hline 69 & $0 \times 00240000$ & C & Endcap \\
\hline 70 & 0x0024000b & $\mathrm{C}$ & Endcap \\
\hline 71 & 0x0024010a & C & Endcap \\
\hline 72 & 0x00240101 & $\mathrm{C}$ & Endcap \\
\hline 73 & 0x00240102 & $\mathrm{C}$ & Endcap \\
\hline 74 & 0x00240001 & $\mathrm{C}$ & Endcap \\
\hline 75 & $0 \mathrm{x} 00240002$ & $\mathrm{C}$ & Endcap \\
\hline 76 & 0x0024000a & C & Endcap \\
\hline 77 & 0x00240009 & $\mathrm{C}$ & Endcap \\
\hline 78 & 0x00240109 & $\mathrm{C}$ & Endcap \\
\hline 79 & 0x00240108 & $\mathrm{C}$ & Endcap \\
\hline 80 & 0x00240005 & $\mathrm{C}$ & Endcap \\
\hline 81 & 0x00240103 & C & Endcap \\
\hline 82 & $0 \mathrm{x} 00240003$ & $\mathrm{C}$ & Endcap \\
\hline 83 & 0x00240008 & $\mathrm{C}$ & Endcap \\
\hline 84 & 0x00240107 & $\mathrm{C}$ & Endcap \\
\hline 85 & 0x00240104 & $\mathrm{C}$ & Endcap \\
\hline 86 & 0x00240106 & $\mathrm{C}$ & Endcap \\
\hline 87 & 0x00240004 & $\mathrm{C}$ & Endcap \\
\hline 88 & $0 \mathrm{x} 00240007$ & $\mathrm{C}$ & Endcap \\
\hline 89 & 0x00240105 & C & Endcap \\
\hline 90 & 0x00240006 & $\mathrm{C}$ & Endcap \\
\hline
\end{tabular}




\section{Appendix G FTK Tower ID}

The FTK simulation software identifies the 64 towers by number ( 0 to 63$)$ as shown in Table 18 . FTK tower partitions are shown in Figure 2(a) and Figure 2(b). Towers 0 to 15 cover the C-Side Endcap, towers 16 to 31 cover the C-Side Barrel, towers 32 to 47 cover the A-Side Barrel, and lastly towers 48 to 63 cover the A-Side Endcap. Note that the FTK tower 0 does not correspond to $\phi 00$ but to $\phi 15$.

\begin{tabular}{c||c|c|c|c|}
\hline & C-Side Endcap & C-Side Barrel & A-Side Barrel & A-Side Endcap \\
\hline$\phi 00$ & 1 & 17 & 33 & 49 \\
\hline$\phi 01$ & 2 & 18 & 34 & 50 \\
\hline$\phi 02$ & 3 & 19 & 35 & 51 \\
\hline$\phi 03$ & 4 & 20 & 36 & 52 \\
\hline$\phi 04$ & 5 & 21 & 37 & 53 \\
\hline$\phi 05$ & 6 & 22 & 38 & 54 \\
\hline$\phi 06$ & 7 & 23 & 39 & 55 \\
\hline$\phi 07$ & 8 & 24 & 40 & 56 \\
\hline$\phi 08$ & 9 & 25 & 41 & 57 \\
\hline$\phi 09$ & 10 & 26 & 42 & 58 \\
\hline$\phi 10$ & 11 & 27 & 43 & 59 \\
\hline$\phi 11$ & 12 & 28 & 44 & 60 \\
\hline$\phi 12$ & 13 & 29 & 45 & 61 \\
\hline$\phi 13$ & 14 & 30 & 46 & 62 \\
\hline$\phi 14$ & 15 & 31 & 47 & 63 \\
\hline$\phi 15$ & 0 & 16 & 32 & 48 \\
\hline
\end{tabular}

Table 18: FTK Tower ID and Tower Location. 


\section{Appendix H SLINK - FPGA Assignments}

The mapping between the SLINK fibers and the Data Formatter FPGAs are summarized in Table 19 and Table 20. This arrangement has been selected to minimize data traffic in the Data Formatter system.

\begin{tabular}{|c|c|c|}
\hline Tower ID & Pixel & SCT \\
\hline 0 & 0x00120205 (45) 0x00130105 (17) & 0x00220001 (27) 0x00240004 (87) \\
\hline 1 & 0x00120220 (59) 0x00130307 (33) & 0x00220009 (33) 0x00240105 (89) \\
\hline 2 & 0x00130310 (36) 0x00130319 (44) & 0x00240101 (72) 0x00240103 (81) \\
\hline 3 & 0x00120218 (57) 0x00121616 (67) & 0x00220006 (41) 0x00240104 (85) \\
\hline 4 & 0x00120216 (55) 0x00130315 (40) & 0x00240100 (68) 0x00240102 (73) \\
\hline 5 & 0x00130108 (20) 0x00130316 (41) & $0 x 00240109(78)$ \\
\hline 6 & 0x00130109 (21) 0x00130112 (24) & 0x00240106 (86) 0x00240107 (84) \\
\hline 7 & 0x00120214 (53) 0x00121614 (65) 0x00130114 (25) & 0x0024010a (71) \\
\hline 8 & 0x00111715 (110) 0x00130117 (28) & 0x00220102 (35) 0x00240108 (79) \\
\hline 9 & 0x00120211 (51) 0x00130118 (29) & 0x00220109 (37) 0x00240006 (90) \\
\hline 10 & 0x00121611 (63) 0x00130005 (1) & 0x00240008 (83) 0x0024000a (76) \\
\hline 11 & 0x00120209 (49) 0x00130008 (4) & 0x00220106 (43) 0x00240009 (77) \\
\hline 12 & 0x00112507 (87) 0x00130009 (5) & 0x00240007 (88) 0x0024000b (70) \\
\hline 13 & 0x00130012 (8) 0x00130014 (9) & 0x00240003 (82) \\
\hline 14 & 0x00130017 (12) 0x00130018 (13) & 0x00240001 (74) 0x00240005 (80) \\
\hline 15 & 0x00120207 (47) 0x00121609 (61) & 0x00240000 (69) 0x00240002 (75) \\
\hline 16 & 0x00111721 (116) 0x00112421 (84) & 0x00220004 (31) \\
\hline 17 & 0x00111706 (102) 0x00112512 (92) & $0 \times 00220005(1)$ \\
\hline 18 & 0x00111806 (118) 0x00111808 (120) & 0x00220007 (3) \\
\hline 19 & 0x00112515 (94) 0x00130311 (37) & 0x00220008 (5) \\
\hline 20 & 0x00111708 (104) 0x00112517 (96) & 0x0022000a (7) \\
\hline 21 & 0x00111710 (106) 0x00112519 (98) & 0x00220100 (9) \\
\hline 22 & 0x00111712 (108) 0x00112521 (100) & 0x00220101 (11) \\
\hline 23 & 0x00111810 (122) 0x00111812 (124) & $0 \times 00220103(13)$ \\
\hline 24 & 0x00111717 (112) 0x00112406 (70) 0x00112408 (72) & 0x00220104 (15) \\
\hline 25 & 0x00112505 (85) 0x00130121 (32) & 0x00220105 (17) \\
\hline 26 & 0x00111815 (126) 0x00112410 (74) & $0 \times 00220107(19)$ \\
\hline 27 & 0x00111817 (128) 0x00112509 (89) & 0x00220108 (21) \\
\hline 28 & 0x00112412 (76) 0x00112415 (78) & 0x0022010a (23) \\
\hline 29 & 0x00111719 (114) 0x00112417 (80) & $0 \times 00220000(25)$ \\
\hline 30 & 0x00111819 (130) 0x00112419 (82) & 0x00220002 (39) \\
\hline 31 & 0x00111821 (132) 0x00130021 (16) & $0 \times 00220003(29)$ \\
\hline
\end{tabular}

Table 19: ROD assignment for C-Side FTK tower (Tower0-31). The numbers noted in the brackets are ROD IDs, corresponding to numbers on Figure 29, and Figure 31. 


\begin{tabular}{|c|c|c|}
\hline Tower ID & Pixel & SCT \\
\hline 32 & 0x00111720 (115) 0x00112420 (83) & $0 \times 00210004(32)$ \\
\hline 33 & 0x00111705 (101) 0x00112511 (91) & $0 \times 00210005(2)$ \\
\hline 34 & 0x00111805 (117) 0x00111807 (119) & 0x00210007 (4) \\
\hline 35 & 0x00112514 (93) 0x00130312 (38) & $0 \times 00210008(6)$ \\
\hline 36 & 0x00111707 (103) 0x00112516 (95) & 0x0021000a (8) \\
\hline 37 & 0x00111709 (105) 0x00112518 (97) & 0x00210100 (10) \\
\hline 38 & 0x00111711 (107) 0x00112520 (99) & $0 \times 00210101(12)$ \\
\hline 39 & 0x00111809 (121) 0x00111811 (123) & $0 \times 00210103(14)$ \\
\hline 40 & 0x00111716 (111) 0x00112405 (69) 0x00112407 (71) & $0 \times 00210104(16)$ \\
\hline 41 & 0x00112506 (86) 0x00130120 (31) & $0 \times 00210105(18)$ \\
\hline 42 & 0x00111814 (125) 0x00112409 (73) & $0 \times 00210107(20)$ \\
\hline 43 & 0x00111816 (127) 0x00112510 (90) & 0x00210108 (22) \\
\hline 44 & 0x00112411 (75) 0x00112414 (77) & 0x0021010a (24) \\
\hline 45 & 0x00111718 (113) 0x00112416 (79) & $0 \times 00210000(26)$ \\
\hline 46 & 0x00111818 (129) 0x00112418 (81) & $0 \times 00210002(40)$ \\
\hline 47 & 0x00111820 (131) 0x00130020 (15) & 0x00210003 (30) \\
\hline 48 & 0x00120206 (46) 0x00130106 (18) & 0x00210001 (28) 0x00230107 (62) \\
\hline 49 & 0x00120221 (60) 0x00130308 (34) & $0 \times 00210009(34) 0 \times 00230005(66)$ \\
\hline 50 & 0x00130309 (35) 0x00130318 (43) & 0x00230007 (60) 0x00230009 (55) \\
\hline 51 & 0x00120219 (58) 0x00121617 (68) & $0 \times 00210006(42) 0 \times 00230006(64)$ \\
\hline 52 & 0x00120217 (56) 0x00130314 (39) & 0x00230008 (56) 0x0023000a (48) \\
\hline 53 & 0x00130107 (19) 0x00130317 (42) & $0 \times 00230001(49)$ \\
\hline 54 & 0x00130110 (22) 0x00130111 (23) & $0 \times 00230003(57) 0 x 00230004(61)$ \\
\hline 55 & 0x00120215 (54) 0x00121615 (66) 0x00130115 (26) & 0x00230000 (45) \\
\hline 56 & 0x00111714 (109) 0x00130116 (27) & $0 \times 00210102(36) 0 x 00230002(50)$ \\
\hline 57 & 0x00120212 (52) 0x00130119 (30) & 0x00210109 (38) 0x00230105 (65) \\
\hline 58 & 0x00121612 (64) 0x00130006 (2) & 0x00230101 (51) 0x00230103 (58) \\
\hline 59 & 0x00120210 (50) 0x00130007 (3) & 0x00210106 (44) 0x00230102 (52) \\
\hline 60 & 0x00112508 (88) 0x00130010 (6) & 0x00230100 (46) 0x00230104 (63) \\
\hline 61 & 0x00130011 (7) 0x00130015 (10) & $0 \times 00230108(59)$ \\
\hline 62 & 0x00130016 (11) 0x00130019 (14) & 0x00230106 (67) 0x0023010a (53) \\
\hline 63 & 0x00120208 (48) 0x00121610 (62) & 0x00230109 (54) 0x0023010b (47) \\
\hline
\end{tabular}

Table 20: ROD assignment for A-Side FTK tower (Tower32-63). The numbers noted in the brackets are ROD IDs, corresponding to numbers on Figure 28 and Figure 30. 


\section{Appendix I Pixel and SCT Data Format}

\section{I.1 S-Link Header and Trailer}

The data format of Pixel and SCT ROD output links are summarized in the ROD User Manual [12]. ROD outputs use the SLINK protocol.

\begin{tabular}{|c|c|c|}
\hline Word & Contents & Comment \\
\hline 0 & OXB0F00000+ UCTRL & Beginning of fragment marker \\
\hline 1 & 0XEE1234EE & Start of header \\
\hline 2 & $0 \times 9$ & Header size \\
\hline 3 & $0 \times 30100000$ & Format Version Number (Ver 3.1) \\
\hline 4 & $\begin{array}{l}\text { 0x001XMMMM Pixel } \\
\text { 0x002XMMMM SCT }\end{array}$ & $\begin{array}{l}\text { Source Identifier } \\
M=\text { Module ID } \quad X=\text { LS Nibble of Sub-detector ID }\end{array}$ \\
\hline 5 & OxTTSSSSSS & 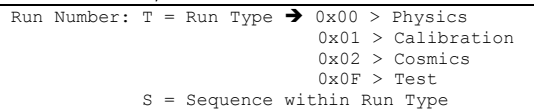 \\
\hline 6 & 0XEELLLLLL & Extended Level 1 ID: $E=E C R$ ID, $L=$ L1ID \\
\hline 7 & $0 \times 00000 \mathrm{BBB}$ & Bunch Counter ID \\
\hline 8 & $0 \times 000000 \mathrm{AA}$ & ATLAS Level 1 Trigger Type \\
\hline 9 & OX00RR000T & Detector Event Type $\mathrm{R}=$ ROD or $\mathrm{T}=\mathrm{TIM}$ \\
\hline
\end{tabular}

Figure 44: ROD Event Header. Refer Table 21 for further details of Source ID words.

\begin{tabular}{|c||c|}
\hline 0x0013XXXX & Pixel Barrel B-layer \\
\hline 0x0011XXXX & Pixel Barrel L1 and L2 \\
\hline 0x0012XXXX & Pixel Endcap disks \\
\hline \hline 0x0021XXXX & SCT A Side Barrel layers \\
\hline 0x0022XXXx & SCT C Side Barrel layers \\
\hline 0x0023XXXX & SCT A Side Endcap disks \\
\hline 0x0024XXXX & SCT C Side Endcap disks \\
\hline
\end{tabular}

Table 21: Source IDs (See Figure 44).

\begin{tabular}{|c|l|l|}
\hline Word & Contents & Comment \\
\hline 0 & Event Fragment Error Flags & Status 1: Bit error see EFB errors [31:0] (Table 31) \\
\hline 1 & $\begin{array}{l}\text { Error Count and } \\
\text { Static Error Flags }\end{array}$ & Status 2: Count of words with error [15:0] \\
TIM OK, BOC OK and ROL Status
\end{tabular}

Figure 45: ROD event trailer 


\section{I.2 Pixel Raw Data}

The Pixel-ROD data words are 32 bits wide, which consist of series of data for individual FE readout modules that connected to the ROD. Since only the header words are reformatted by the router FPGAs on the RODs, refer Figure 47 for the header word definition for the FTK inputs.

The "Link Number" in the header word definition indicates the module ID of the data packet. It is translated into the "FMT" ID and "LINK" ID. Note that the these definition depends on the configuration of the readout speed $(40 \mathrm{MHz}$ or $80 \mathrm{MHz})$. Generally speaking all layer-2 RODs use $40 \mathrm{MHz}$ mode, the layer-1, B-layer and disc RODs use the $80 \mathrm{MHz}$ readout mode. In addition the B-layer modules send their data on two links (resulting in an effective $160 \mathrm{Mb} / \mathrm{s}$ ) whereas all other modules have only one link.

\begin{tabular}{|l|l|}
\hline \multicolumn{1}{|c|}{ Name } & Bits [31:0] \\
\hline Header & 001PxxxxxxxxAAAAMMMMLLLLBBBBBBBB \\
\hline Trailer & 0102HVxxxxxxxxxxxxxxxxxxxxxx \\
\hline Hit & 100xFFFFTTTTTTXXXCCCCRRRRRRRR \\
\hline FE Flag Error (Old) & 0000 FFFFxxxxxxxxx11110FFFFEEEE \\
\hline FE Flag Error (New) & 0001 FFFFxxx11111eeeeeeeEEEEEEEE \\
\hline Raw Data & 011DDDDDDDDDDDDDDDDDDDDDDDDDDDD \\
\hline Time Out Data & 00100000000000000000000000000 \\
\hline
\end{tabular}

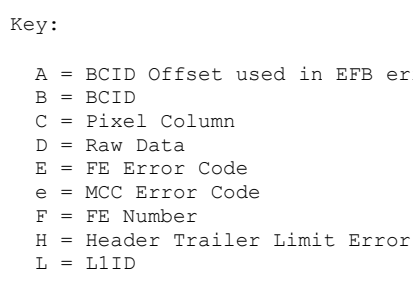

Figure 46: Pixel RAW data definition. Note that the header word will eventually be reformatted in the ROD, which is shown Figure 47.

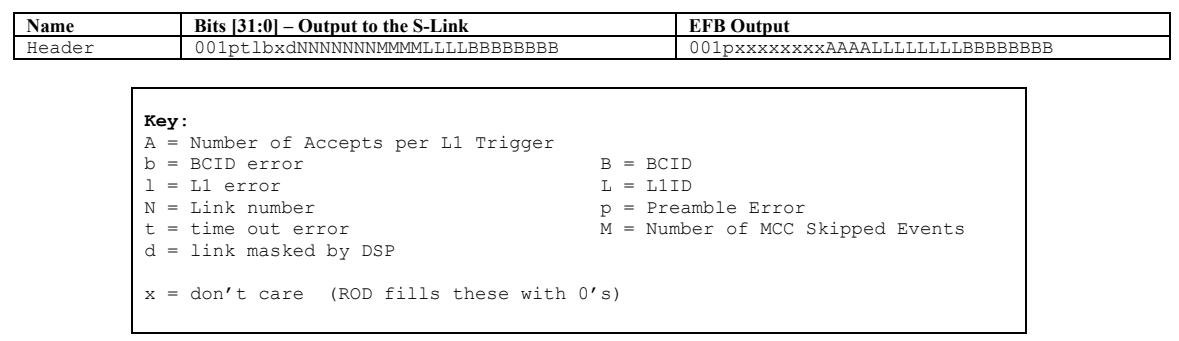

Figure 47: Header words in Pixel output.

\section{I.3 SCT Raw Data}

All event data is in 16-bit words packed in 32-bit frames. Since only the header words are reformatted by the router FPGAs on the RODs, refer Figure 49 for the header word definition for the FTK inputs.

The SCT has two readout modes: condensed and expanded. The expanded mode was used for 2012 operations while the condensed mode will be used for higher luminosity runs going forward. The expanded mode supports multiple edge modes (e.g. the neighboring three bunch crossings). Condensed mode uses only the ("01X") edge mode. 


\begin{tabular}{|c|c|}
\hline Name & Bits $[15: 0]$ or $[31: 16]$ \\
\hline Header & 001 pLLLLBBBBBBBB \\
\hline Trailer & 010zhvxxxxxxxxxx \\
\hline 1 hit condensed & 1FFFFCCCCCCCXfxo \\
\hline 2 hits condensed & 1FFFFCCCCCCCsfx1 \\
\hline 1st hit cluster expanded & 1FFFFCCCCCCCODDD \\
\hline 1 hit cluster expanded & 1Xxxxxxx0xxx1DDD \\
\hline 2 hit cluster expanded & 1Xxxxxxx1DDD1DDD \\
\hline Flagged error & 000XXXXXXFFFFEEE \\
\hline Raw data & 011nnnxxWWWWWWWW \\
\hline
\end{tabular}

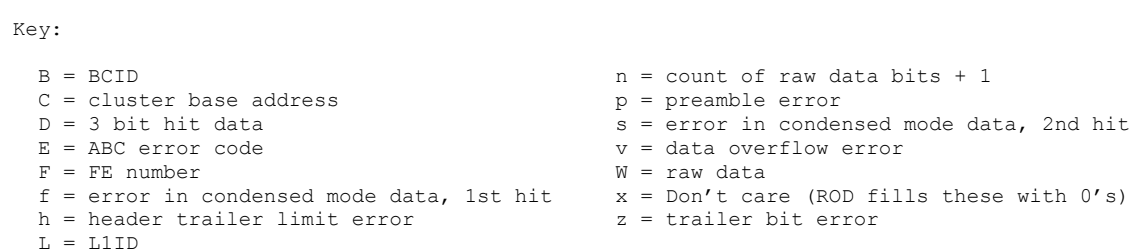

Figure 48: SCT RAW data definition. Note that the header word will eventually be reformatted in the ROD, which is shown Figure 49.

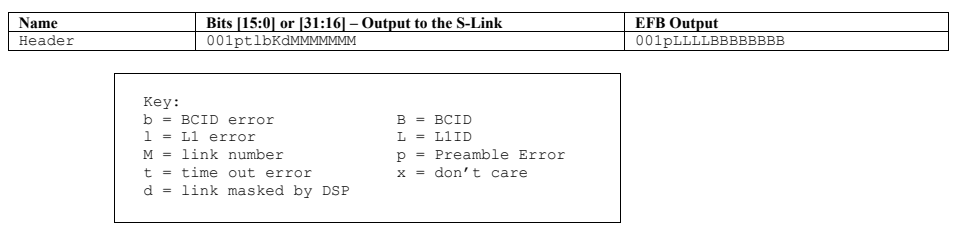

Figure 49: Header words in SCT output. The "Link Number" in the Table indicates the modules ID and layers of the SCT doublet.

\section{I.4 Number of SLINK Words}

We studied the number of words per ROD as part of our input data volume study using the actual collision data with $\sqrt{s}=8 \mathrm{TeV},\langle\mu\rangle=30.0$, and $50 \mathrm{~ns}$ bunch spacing. Refer Section 8.2.1 for details of the data-sample.

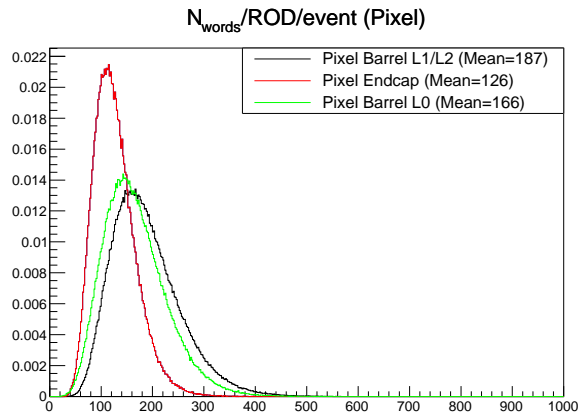

(a)

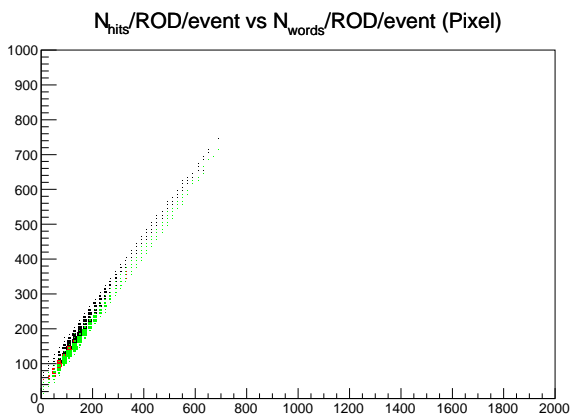

(b)

Figure 50: Input data volume distribution for Pixel RODs. (a) shows number of 32-bit S-Link words per ROD per event distribution. (b) shows correlation between the $N_{\text {hits }}$ and $N_{\text {words }}$ (Refer Figure 18). 


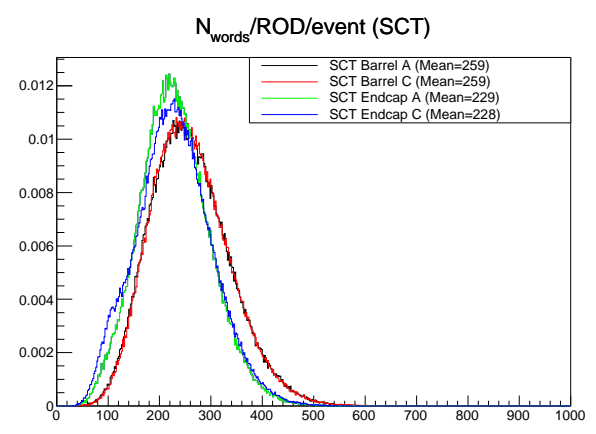

(a)

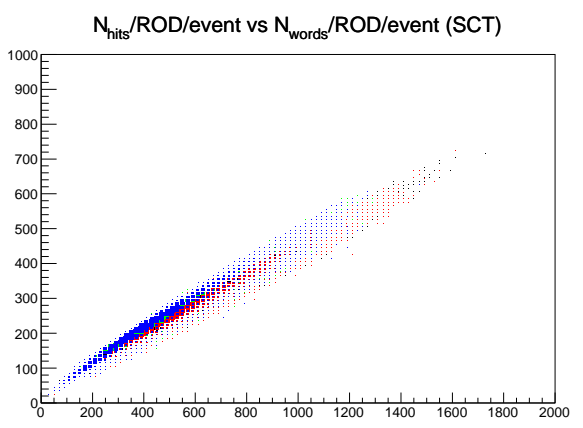

(b)

Figure 51: Input data volume distribution for SCT RODs. (b) shows number of 32-bit S-Link words per ROD per event distribution. (c) shows correlation between the $N_{\text {hits }}$ and $N_{\text {words }}$ (Refer Figure 18). 


\section{Appendix J Tails in the Number of Hits per Module}

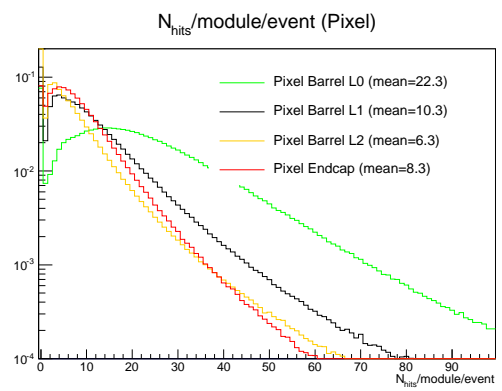

(a)

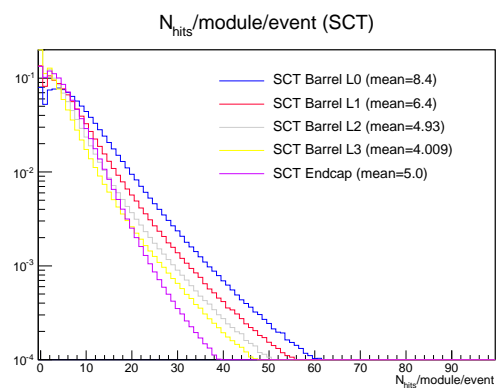

(b)

Figure 52: $N_{\text {hits }}$ per module per event distribution in log scale. Details of this tail component are shown in Tables 22 and 23. LHC operating conditions were $\sqrt{s}=8 \mathrm{TeV},\langle\mu\rangle=30.0$, with a 50 ns bunch spacing.

\begin{tabular}{c||c|c|c|c}
\hline & $N_{\text {hits }} \geq 5$ & $N_{\text {hits }} \geq 10$ & $N_{\text {hits }} \geq 15$ & $N_{\text {hits }} \geq 20$ \\
\hline pixel (Barrel L0) & 87.8 & 76.4 & 62.5 & 48.5 \\
\hline pixel (Barrel L1) & 67.6 & 40.6 & 23.2 & 13.5 \\
\hline pixel (Barrel L2) & 62.9 & 29.6 & 14.8 & 8.1 \\
\hline pixel (Endcap) & 37.8 & 18.7 & 8.7 & 4.3 \\
\hline
\end{tabular}

Table 22: Tail components of the $N_{\text {hits }}$ per module per event distribution for Pixel shown in percentage term. LHC operating conditions were $\sqrt{s}=8 \mathrm{TeV},\langle\mu\rangle=30.0$, with a $50 \mathrm{~ns}$ bunch spacing.

\begin{tabular}{c||c|c|c|c}
\hline & $N_{\text {hits }} \geq 5$ & $N_{\text {hits }} \geq 10$ & $N_{\text {hits }} \geq 15$ & $N_{\text {hits }} \geq 20$ \\
\hline SCT (Barrel L0) & 63.8 & 32.2 & 15.4 & 7.7 \\
\hline SCT (Barrel L1) & 49.5 & 21.0 & 9.4 & 4.6 \\
\hline SCT (Barrel L2) & 37.9 & 14.2 & 6.1 & 3.0 \\
\hline SCT (Barrel L3) & 29.4 & 10.3 & 4.4 & 2.2 \\
\hline SCT (Endcap) & 43.5 & 13.6 & 4.2 & 1.5 \\
\hline
\end{tabular}

Table 23: Tail components of the $N_{\text {hits }}$ per module per event distribution for SCT shown in percentage term. LHC operating conditions were $\sqrt{s}=8 \mathrm{TeV},\langle\mu\rangle=30.0$, with a 50 ns bunch spacing. 


\section{Appendix K Downstream Data Flow}

In Table 24 and Table 25 we summarize the dataflow from the Data Formatter to the 64 individual FTK towers. This analysis was done with real collision data collected from runs taken at $\sqrt{s}=8 \mathrm{TeV},\langle\mu\rangle=30.0$, with a $50 \mathrm{~ns}$ bunch spacing. Please refer the $N_{\text {hits }}$ definition in Section 8.2.2. The numbers are extrapolated into $\sqrt{s}=14 \mathrm{TeV},\langle\mu\rangle=70.0$, and $25 \mathrm{~ns}$ bunch spacing conditions in Table K and Table K.

\begin{tabular}{|c||c|c|c|c||c|c|c|c|c||c|c|}
\hline Tower & P-B-L0 & P-B-L1 & P-B-L2 & P-EC & S-B-L0 & S-B-L1 & S-B-L2 & S-B-L3 & S-EC & $N_{\text {px1 }}$ & $N_{\text {SCT }}$ \\
\hline 0 & 414 & 283 & 199 & 130 & 191 & 96 & 47 & 0 & 1097 & 1026 & 1431 \\
\hline 1 & 486 & 254 & 199 & 114 & 189 & 119 & 47 & 0 & 1101 & 1052 & 1456 \\
\hline 2 & 493 & 255 & 172 & 105 & 187 & 95 & 47 & 0 & 1160 & 1025 & 1489 \\
\hline 3 & 500 & 323 & 166 & 115 & 187 & 119 & 47 & 0 & 1165 & 1104 & 1518 \\
\hline 4 & 482 & 247 & 180 & 124 & 189 & 96 & 47 & 0 & 1102 & 1033 & 1434 \\
\hline 5 & 483 & 313 & 186 & 124 & 189 & 121 & 47 & 0 & 1095 & 1105 & 1452 \\
\hline 6 & 319 & 258 & 175 & 133 & 189 & 96 & 47 & 0 & 1118 & 885 & 1450 \\
\hline 7 & 467 & 257 & 173 & 134 & 190 & 119 & 47 & 0 & 1108 & 1031 & 1465 \\
\hline 8 & 487 & 311 & 207 & 134 & 189 & 96 & 46 & 0 & 1042 & 1139 & 1374 \\
\hline 9 & 474 & 245 & 206 & 132 & 190 & 121 & 46 & 0 & 1052 & 1057 & 1409 \\
\hline 10 & 469 & 255 & 160 & 131 & 193 & 98 & 47 & 0 & 1129 & 1015 & 1466 \\
\hline 11 & 437 & 315 & 151 & 132 & 192 & 118 & 47 & 0 & 1156 & 1036 & 1513 \\
\hline 12 & 451 & 240 & 205 & 131 & 175 & 97 & 47 & 0 & 1097 & 1028 & 1416 \\
\hline 13 & 475 & 299 & 204 & 130 & 175 & 122 & 48 & 0 & 1101 & 1108 & 1446 \\
\hline 14 & 317 & 245 & 136 & 113 & 192 & 96 & 47 & 0 & 1155 & 811 & 1490 \\
\hline 15 & 411 & 238 & 144 & 121 & 192 & 119 & 47 & 0 & 1163 & 914 & 1522 \\
\hline 16 & 346 & 327 & 266 & 0 & 329 & 293 & 331 & 315 & 0 & 939 & 1269 \\
\hline 17 & 416 & 290 & 267 & 0 & 327 & 354 & 331 & 316 & 0 & 974 & 1328 \\
\hline 18 & 419 & 292 & 234 & 0 & 309 & 292 & 331 & 316 & 0 & 945 & 1248 \\
\hline 19 & 426 & 370 & 227 & 0 & 309 & 367 & 330 & 298 & 0 & 1023 & 1304 \\
\hline 20 & 436 & 276 & 225 & 0 & 325 & 295 & 319 & 305 & 0 & 937 & 1245 \\
\hline 21 & 437 & 349 & 225 & 0 & 329 & 371 & 330 & 313 & 0 & 1011 & 1343 \\
\hline 22 & 245 & 299 & 225 & 0 & 328 & 295 & 331 & 315 & 0 & 769 & 1270 \\
\hline 23 & 344 & 297 & 225 & 0 & 325 & 373 & 331 & 317 & 0 & 867 & 1346 \\
\hline 24 & 391 & 357 & 270 & 0 & 328 & 299 & 332 & 317 & 0 & 1017 & 1276 \\
\hline 25 & 406 & 282 & 282 & 0 & 330 & 372 & 329 & 318 & 0 & 969 & 1349 \\
\hline 26 & 400 & 293 & 216 & 0 & 331 & 295 & 329 & 316 & 0 & 909 & 1272 \\
\hline 27 & 367 & 360 & 193 & 0 & 331 & 355 & 331 & 315 & 0 & 920 & 1333 \\
\hline 28 & 383 & 274 & 253 & 0 & 314 & 282 & 332 & 308 & 0 & 910 & 1236 \\
\hline 29 & 410 & 346 & 272 & 0 & 311 & 370 & 333 & 309 & 0 & 1028 & 1323 \\
\hline 30 & 273 & 283 & 199 & 0 & 327 & 295 & 331 & 316 & 0 & 755 & 1269 \\
\hline 31 & 342 & 275 & 213 & 0 & 330 & 366 & 332 & 314 & 0 & 830 & 1341 \\
\hline \hline
\end{tabular}

Table 24: The number of hits in the output stream from DF to AUX or SSB. These numbers are extracted from a run with $\sqrt{s}=8 \mathrm{TeV}$ and $\langle\mu\rangle=30$ (Run:214523, LB:153). This Table references Tower IDs 0 to 31 for the C-Side. Number of SCT hits are counted with "edge mode" emulation. 


\begin{tabular}{|c||c|c|c|c||c|c|c|c|c||c|c|}
\hline Tower & P-B-L0 & P-B-L1 & P-B-L2 & P-EC & S-B-L0 & S-B-L1 & S-B-L2 & S-B-L3 & S-EC & $N_{\text {pxx }}$ & $N_{\text {SCT }}$ \\
\hline 32 & 347 & 336 & 274 & 0 & 329 & 296 & 335 & 314 & 0 & 958 & 1274 \\
\hline 33 & 418 & 290 & 274 & 0 & 328 & 371 & 332 & 315 & 0 & 983 & 1347 \\
\hline 34 & 420 & 292 & 235 & 0 & 327 & 295 & 330 & 316 & 0 & 947 & 1268 \\
\hline 35 & 426 & 369 & 235 & 0 & 327 & 367 & 330 & 305 & 0 & 1030 & 1329 \\
\hline 36 & 436 & 275 & 238 & 0 & 328 & 294 & 330 & 305 & 0 & 948 & 1256 \\
\hline 37 & 413 & 350 & 230 & 0 & 331 & 369 & 331 & 312 & 0 & 993 & 1343 \\
\hline 38 & 222 & 299 & 224 & 0 & 332 & 296 & 332 & 313 & 0 & 745 & 1273 \\
\hline 39 & 367 & 287 & 211 & 0 & 329 & 372 & 332 & 313 & 0 & 864 & 1346 \\
\hline 40 & 414 & 348 & 257 & 0 & 329 & 296 & 332 & 315 & 0 & 1018 & 1271 \\
\hline 41 & 407 & 282 & 282 & 0 & 330 & 370 & 332 & 316 & 0 & 971 & 1347 \\
\hline 42 & 379 & 291 & 221 & 0 & 331 & 296 & 333 & 315 & 0 & 892 & 1276 \\
\hline 43 & 344 & 358 & 199 & 0 & 332 & 366 & 330 & 314 & 0 & 902 & 1342 \\
\hline 44 & 383 & 273 & 254 & 0 & 330 & 292 & 330 & 306 & 0 & 911 & 1257 \\
\hline 45 & 410 & 347 & 273 & 0 & 327 & 366 & 330 & 307 & 0 & 1030 & 1330 \\
\hline 46 & 273 & 284 & 199 & 0 & 328 & 292 & 329 & 305 & 0 & 756 & 1254 \\
\hline 47 & 343 & 286 & 227 & 0 & 330 & 366 & 333 & 305 & 0 & 855 & 1334 \\
\hline 48 & 439 & 312 & 200 & 131 & 191 & 96 & 47 & 0 & 1105 & 1082 & 1440 \\
\hline 49 & 495 & 254 & 200 & 124 & 191 & 121 & 47 & 0 & 1111 & 1072 & 1470 \\
\hline 50 & 500 & 257 & 173 & 132 & 190 & 96 & 47 & 0 & 1162 & 1061 & 1495 \\
\hline 51 & 505 & 322 & 172 & 125 & 190 & 119 & 46 & 0 & 1157 & 1125 & 1512 \\
\hline 52 & 515 & 257 & 194 & 126 & 191 & 95 & 46 & 0 & 1086 & 1093 & 1418 \\
\hline 53 & 492 & 322 & 194 & 134 & 192 & 120 & 47 & 0 & 1094 & 1142 & 1453 \\
\hline 54 & 248 & 259 & 166 & 123 & 191 & 95 & 46 & 0 & 1156 & 796 & 1489 \\
\hline 55 & 418 & 247 & 138 & 123 & 191 & 120 & 47 & 0 & 1167 & 926 & 1526 \\
\hline 56 & 486 & 310 & 174 & 131 & 159 & 95 & 47 & 0 & 1098 & 1101 & 1399 \\
\hline 57 & 478 & 255 & 199 & 124 & 158 & 120 & 47 & 0 & 1095 & 1056 & 1420 \\
\hline 58 & 451 & 253 & 163 & 114 & 191 & 95 & 48 & 0 & 1148 & 981 & 1482 \\
\hline 59 & 419 & 313 & 151 & 121 & 189 & 119 & 46 & 0 & 1146 & 1004 & 1501 \\
\hline 60 & 458 & 240 & 186 & 122 & 190 & 95 & 46 & 0 & 1080 & 1005 & 1410 \\
\hline 61 & 481 & 289 & 198 & 130 & 191 & 119 & 47 & 0 & 1097 & 1099 & 1454 \\
\hline 62 & 319 & 238 & 122 & 129 & 189 & 96 & 47 & 0 & 1164 & 808 & 1496 \\
\hline 63 & 435 & 252 & 165 & 130 & 189 & 120 & 47 & 0 & 1175 & 981 & 1531 \\
\hline \hline
\end{tabular}

Table 25: The number of hits in the output stream from DF to AUX or SSB. These numbers are extracted from a run with $\sqrt{s}=8 \mathrm{TeV}$ and $\langle\mu\rangle=30$ (Run:214523, LB:153). This Table references Tower IDs 32 to 63 for the A-Side. Number of SCT hits are counted with "edge mode" emulation. 


\begin{tabular}{|c|c|c|c|c|c|c|c|c|c|c|c|}
\hline Tower & P-B-L0 & P-B-L1 & P-B-L2 & P-EC & S-B-L0 & S-B-L1 & S-B-L2 & S-B-L3 & S-EC & $N_{\mathrm{pxl}}$ & $N_{\mathrm{SCT}}$ \\
\hline 0 & 1401 & 958 & 672 & 441 & 646 & 326 & 158 & 0 & 3712 & 3472 & 4841 \\
\hline 1 & 1645 & 858 & 673 & 384 & 639 & 404 & 159 & 0 & 3726 & 3559 & 4927 \\
\hline 2 & 1668 & 864 & 582 & 355 & 633 & 322 & 157 & 0 & 3925 & 3469 & 5037 \\
\hline 3 & 1690 & 1092 & 563 & 390 & 634 & 403 & 158 & 0 & 3942 & 3735 & 5137 \\
\hline 4 & 1630 & 837 & 610 & 418 & 640 & 326 & 158 & 0 & 3728 & 3495 & 4851 \\
\hline 5 & 1634 & 1057 & 630 & 419 & 640 & 411 & 158 & 0 & 3705 & 3740 & 4913 \\
\hline 6 & 1081 & 872 & 592 & 450 & 640 & 324 & 160 & 0 & 3782 & 2995 & 4906 \\
\hline 7 & 1580 & 869 & 586 & 453 & 643 & 404 & 159 & 0 & 3749 & 3489 & 4955 \\
\hline 8 & 1647 & 1051 & 701 & 454 & 640 & 326 & 157 & 0 & 3525 & 3853 & 4648 \\
\hline 9 & 1604 & 829 & 698 & 446 & 644 & 408 & 157 & 0 & 3558 & 3576 & 4767 \\
\hline 10 & 1588 & 861 & 541 & 442 & 652 & 331 & 158 & 0 & 3819 & 3433 & 4961 \\
\hline 11 & 1478 & 1067 & 512 & 447 & 648 & 399 & 159 & 0 & 3912 & 3504 & 5118 \\
\hline 12 & 1527 & 811 & 695 & 444 & 593 & 327 & 161 & 0 & 3712 & 3476 & 4792 \\
\hline 13 & 1608 & 1012 & 691 & 439 & 593 & 411 & 162 & 0 & 3726 & 3750 & 4892 \\
\hline 14 & 1072 & 829 & 460 & 382 & 648 & 325 & 160 & 0 & 3907 & 2743 & 5040 \\
\hline 15 & 1391 & 804 & 488 & 410 & 650 & 403 & 159 & 0 & 3936 & 3092 & 5148 \\
\hline 16 & 1170 & 1105 & 902 & 0 & 1114 & 992 & 1121 & 1066 & 0 & 3177 & 4292 \\
\hline 17 & 1408 & 981 & 905 & 0 & 1107 & 1197 & 1121 & 1068 & 0 & 3294 & 4493 \\
\hline 18 & 1418 & 987 & 791 & 0 & 1044 & 989 & 1121 & 1068 & 0 & 3196 & 4222 \\
\hline 19 & 1440 & 1252 & 770 & 0 & 1044 & 1241 & 1117 & 1009 & 0 & 3461 & 4411 \\
\hline 20 & 1476 & 933 & 763 & 0 & 1099 & 999 & 1080 & 1032 & 0 & 3171 & 4211 \\
\hline 21 & 1477 & 1182 & 761 & 0 & 1113 & 1254 & 1116 & 1060 & 0 & 3420 & 4543 \\
\hline 22 & 829 & 1012 & 762 & 0 & 1110 & 999 & 1121 & 1066 & 0 & 2603 & 4297 \\
\hline 23 & 1165 & 1006 & 762 & 0 & 1099 & 1261 & 1121 & 1073 & 0 & 2932 & 4554 \\
\hline 24 & 1322 & 1208 & 912 & 0 & 1111 & 1010 & 1123 & 1073 & 0 & 3442 & 4318 \\
\hline 25 & 1373 & 953 & 953 & 0 & 1115 & 1260 & 1112 & 1076 & 0 & 3279 & 4563 \\
\hline 26 & 1354 & 991 & 730 & 0 & 1121 & 998 & 1114 & 1070 & 0 & 3075 & 4304 \\
\hline 27 & 1243 & 1217 & 654 & 0 & 1121 & 1200 & 1121 & 1067 & 0 & 3113 & 4509 \\
\hline 28 & 1297 & 927 & 856 & 0 & 1061 & 954 & 1125 & 1042 & 0 & 3080 & 4182 \\
\hline 29 & 1386 & 1171 & 920 & 0 & 1052 & 1252 & 1127 & 1045 & 0 & 3477 & 4477 \\
\hline 30 & 924 & 957 & 672 & 0 & 1107 & 998 & 1121 & 1068 & 0 & 2553 & 4294 \\
\hline 31 & 1157 & 930 & 720 & 0 & 1116 & 1238 & 1122 & 1063 & 0 & 2807 & 4539 \\
\hline
\end{tabular}

Table 26: The number of hits in output stream from DF to AUX or SSB. The numbers extracted from a run with $\sqrt{s}=8 \mathrm{TeV}$ and $\langle\mu\rangle=30$ (Run:214523, LB:153) and extrapolated into $14 \mathrm{TeV}$ and $\langle\mu\rangle=70$. This Table references Tower IDs 0 to 31 for the C-Side. Number of SCT hits are counted with "edge mode" emulation. 


\begin{tabular}{|c|c|c|c|c|c|c|c|c|c|c|c|}
\hline Tower & P-B-L0 & P-B-L1 & P-B-L2 & P-EC & S-B-L0 & S-B-L1 & S-B-L2 & S-B-L3 & S-EC & $N_{\mathrm{pxl}}$ & $N_{\mathrm{SCT}}$ \\
\hline 32 & 1093 & 1060 & 864 & 0 & 1038 & 933 & 1054 & 989 & 0 & 3016 & 4014 \\
\hline 33 & 1317 & 914 & 864 & 0 & 1033 & 1169 & 1047 & 993 & 0 & 3096 & 4243 \\
\hline 34 & 1322 & 921 & 741 & 0 & 1030 & 931 & 1040 & 994 & 0 & 2985 & 3995 \\
\hline 35 & 1341 & 1163 & 740 & 0 & 1031 & 1155 & 1039 & 960 & 0 & 3244 & 4185 \\
\hline 36 & 1373 & 865 & 749 & 0 & 1033 & 925 & 1039 & 960 & 0 & 2987 & 3958 \\
\hline 37 & 1302 & 1101 & 725 & 0 & 1043 & 1162 & 1042 & 982 & 0 & 3128 & 4229 \\
\hline 38 & 701 & 941 & 705 & 0 & 1045 & 932 & 1046 & 987 & 0 & 2347 & 4010 \\
\hline 39 & 1156 & 903 & 663 & 0 & 1037 & 1173 & 1045 & 987 & 0 & 2722 & 4241 \\
\hline 40 & 1303 & 1095 & 809 & 0 & 1035 & 932 & 1044 & 992 & 0 & 3207 & 4004 \\
\hline 41 & 1282 & 888 & 888 & 0 & 1039 & 1164 & 1045 & 995 & 0 & 3058 & 4242 \\
\hline 42 & 1193 & 918 & 697 & 0 & 1044 & 932 & 1050 & 993 & 0 & 2808 & 4019 \\
\hline 43 & 1085 & 1127 & 628 & 0 & 1045 & 1154 & 1040 & 988 & 0 & 2840 & 4227 \\
\hline 44 & 1208 & 861 & 799 & 0 & 1041 & 918 & 1039 & 963 & 0 & 2868 & 3961 \\
\hline 45 & 1293 & 1092 & 859 & 0 & 1030 & 1152 & 1041 & 966 & 0 & 3244 & 4188 \\
\hline 46 & 861 & 894 & 626 & 0 & 1032 & 921 & 1035 & 961 & 0 & 2380 & 3950 \\
\hline 47 & 1080 & 899 & 714 & 0 & 1040 & 1154 & 1048 & 962 & 0 & 2693 & 4204 \\
\hline 48 & 1383 & 982 & 630 & 412 & 601 & 303 & 149 & 0 & 3482 & 3407 & 4535 \\
\hline 49 & 1558 & 801 & 629 & 391 & 601 & 382 & 148 & 0 & 3499 & 3378 & 4630 \\
\hline 50 & 1574 & 808 & 544 & 417 & 598 & 303 & 147 & 0 & 3660 & 3343 & 4708 \\
\hline 51 & 1591 & 1016 & 543 & 393 & 599 & 374 & 146 & 0 & 3646 & 3543 & 4764 \\
\hline 52 & 1623 & 810 & 612 & 396 & 602 & 301 & 145 & 0 & 3420 & 3441 & 4468 \\
\hline 53 & 1550 & 1015 & 611 & 421 & 605 & 376 & 148 & 0 & 3447 & 3597 & 4576 \\
\hline 54 & 781 & 815 & 524 & 388 & 603 & 300 & 145 & 0 & 3642 & 2508 & 4690 \\
\hline 55 & 1318 & 777 & 434 & 388 & 602 & 379 & 147 & 0 & 3677 & 2917 & 4805 \\
\hline 56 & 1530 & 977 & 547 & 413 & 500 & 301 & 148 & 0 & 3458 & 3467 & 4407 \\
\hline 57 & 1505 & 804 & 628 & 390 & 498 & 377 & 148 & 0 & 3449 & 3327 & 4471 \\
\hline 58 & 1420 & 796 & 514 & 360 & 601 & 301 & 150 & 0 & 3616 & 3090 & 4667 \\
\hline 59 & 1320 & 987 & 474 & 383 & 596 & 374 & 146 & 0 & 3610 & 3164 & 4727 \\
\hline 60 & 1444 & 755 & 584 & 383 & 598 & 298 & 146 & 0 & 3401 & 3166 & 4442 \\
\hline 61 & 1516 & 910 & 625 & 410 & 602 & 375 & 148 & 0 & 3456 & 3461 & 4581 \\
\hline 62 & 1005 & 751 & 384 & 405 & 596 & 303 & 148 & 0 & 3667 & 2546 & 4714 \\
\hline 63 & 1370 & 793 & 521 & 408 & 596 & 379 & 148 & 0 & 3700 & 3092 & 4822 \\
\hline
\end{tabular}

Table 27: The number of hits in the output stream from DF to AUX or SSB. The numbers extracted from a run with $\sqrt{s}=8 \mathrm{TeV}$ and $\langle\mu\rangle=30$ (Run:214523, LB:153) and extrapolated into $14 \mathrm{TeV}$ and $\langle\mu\rangle=70$. This Table references Tower IDs 32 to 63 for the A-Side. Number of SCT hits are counted with "edge mode" emulation. 


\section{Appendix L Data Flow Model}

Each Data Formatter FPGA must determine where each incoming data packet will go. Figure 53 shows the decision making process, which was introduced in Section 8.2.5.

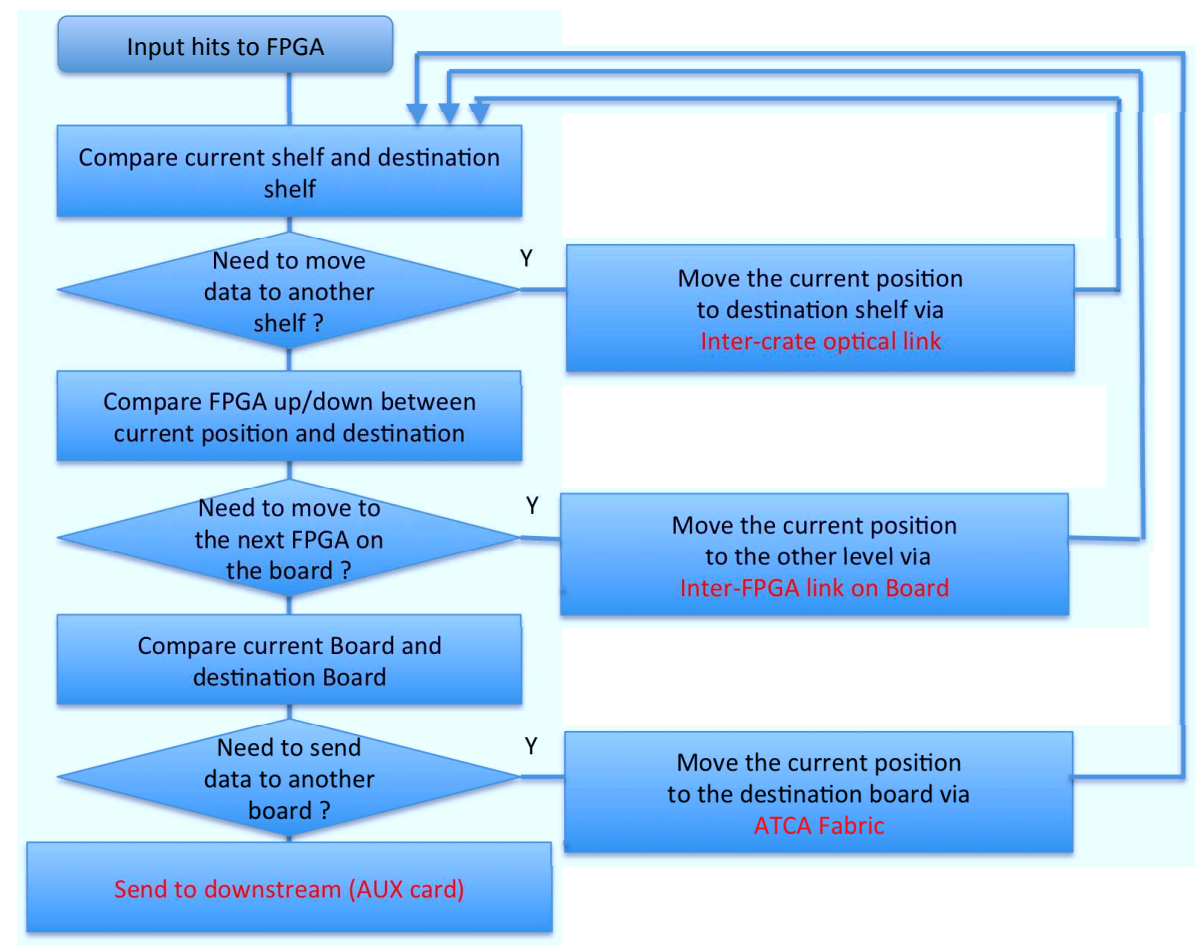

Figure 53: Flowchart of the simulation of data sharing inside the Data Formatter system. 


\section{Appendix M Data Volume Luminosity Dependence}

In this section the data volume is studied as a function of pileup $\langle\mu\rangle$. We use nominal configuration runs and also special high $\langle\mu\rangle$ runs taken in 2012 with $\sqrt{s}=8 \mathrm{TeV}$ are used so that it is possible to measure data volume at high pileup collisions with $\langle\mu\rangle$ up to $\sim 70$. One of the special runs (run 206717) covers $46<\langle\mu\rangle<52$ and the other covers (run 206725) covers $61<\langle\mu\rangle<67$. Only one bunch is filled in run 206717, and two bunches without train structure are filled in run 206725. To compare them without bias due to the operating conditions the following criteria are applied:

- Events are required to be recorded by random trigger.

- Events are required to be corresponding to the first bunch crossings of the train structures (see Figure 56, 57).

- SCT hit timing is required to be "010" or "011" in counting number of SCT raw hits. Hits at both of SCT doublet layers are counted.

The $\langle\mu\rangle$ and data volume have linearity up to $\langle\mu\rangle \sim 70$. Refer the simulation study to see the linearity [3].

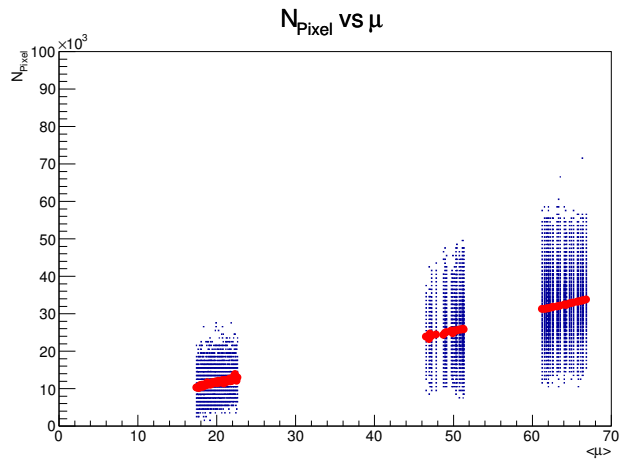

(a)

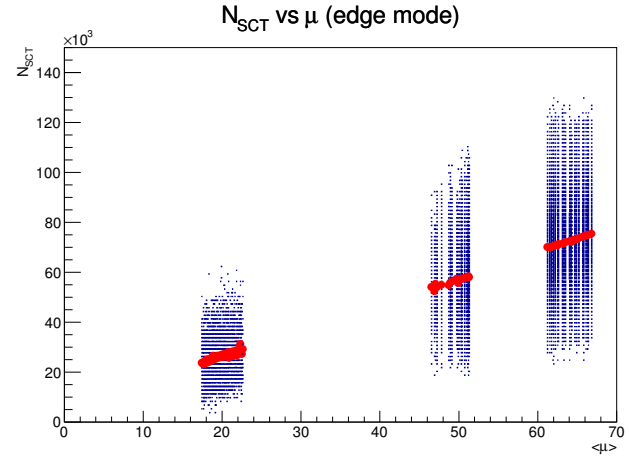

(b)

Figure 54: Data volume dependence on $\langle\mu\rangle$ with $\sqrt{s}=8 \mathrm{TeV}$. (a) Number of Pixel raw hits, and (b) Number of SCT raw hits passing "01X" timing cut (emulating "edge mode" operation). 


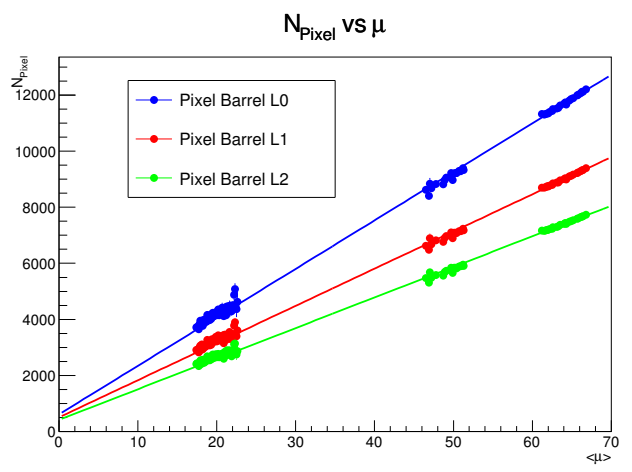

(a)

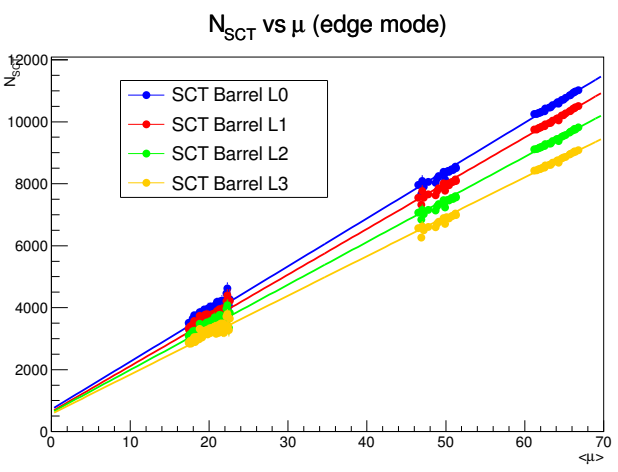

(b)

Figure 55: Data volume dependence on $\langle\mu\rangle$ with $\sqrt{s}=8 \mathrm{TeV}$ at individual Barrel layers. (i.e. Endcap is not included.) (a) Number of Pixel raw hits, and (b) Number of SCT raw hits passing "01X" timing cut (emulating "edge mode" operation).

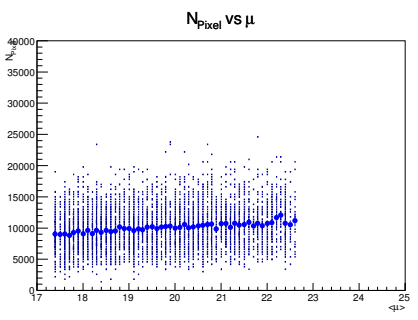

(a)

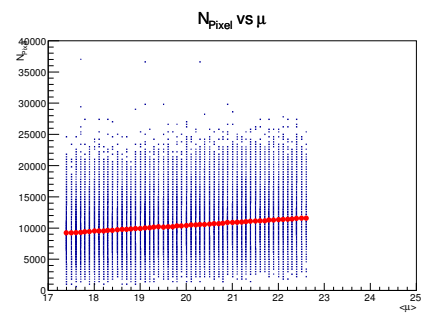

(b)

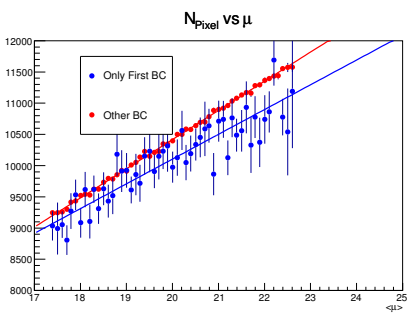

(c)

Figure 56: (a) Number of Pixel hits (barrel) in first collisions of trains and (b) the other collisions. (c) Fitting results with the first polynomial function.

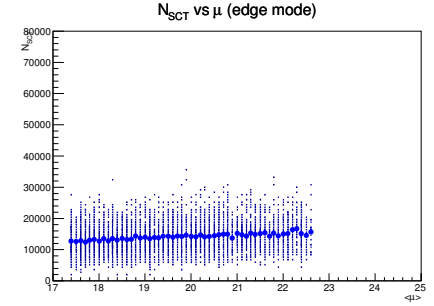

(a)

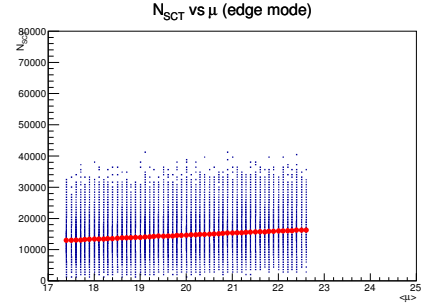

(b)

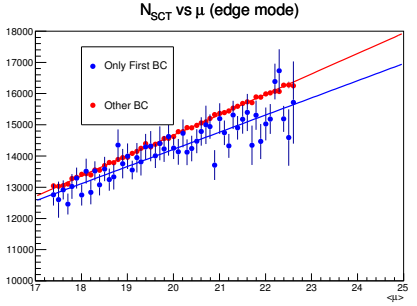

(c)

Figure 57: (a) Number of SCT hits (barrel) in first collisions of trains and (b) the other collisions. (c) Fitting results with the first polynomial function. 


\section{Appendix N Data Volume Monte Carlo Comparison}

We used the following three types of data samples to compare the behavior of $N_{\text {hits }}$ between MC samples and Data.

- Collision data $(\sqrt{s}=8 \mathrm{TeV}, 50 \mathrm{~ns}$ bunch-spacing, $\langle\mu\rangle \sim 20)$, fitted with linear function, shown with closed circles.

- MC samples ( $\sqrt{s}=8 \mathrm{TeV}, 50 \mathrm{~ns}$ bunch-spacing), shown with open circles.

- MC samples ( $\sqrt{s}=14 \mathrm{TeV}, 25 \mathrm{~ns}$ bunch-spacing), shown with closed crosses.

Note that the SCT readout condition is different in the $50 \mathrm{~ns}$ bunch-spacing MC sample $(\sqrt{s}=8 \mathrm{TeV})$ and the $25 \mathrm{~ns}$ bunch-spacing $(\sqrt{s}=14 \mathrm{TeV})$. The readout bunch window configuration is "X1X" for the 50 ns sample, and "01X" (edge modes) for the 25 ns sample. In order to compare the real data and the 50 ns bunch-spacing samples, "X1X" cut is emulated in the offline analysis for the real collision data sample ${ }^{6}$.

Figure 58(a) and Figure 58(b) show the number of detected hits per event as a function of $\langle\mu\rangle$ for pixel hits $\left(N_{\text {Pixel }}\right)$ and SCT hits $\left(N_{\mathrm{SCT}}\right)$, respectively. Figure $59(\mathrm{a})$ and Figure $59(\mathrm{~b})$ show the number of detected hits at individual barrel layers for the pixel and SCT respectively.

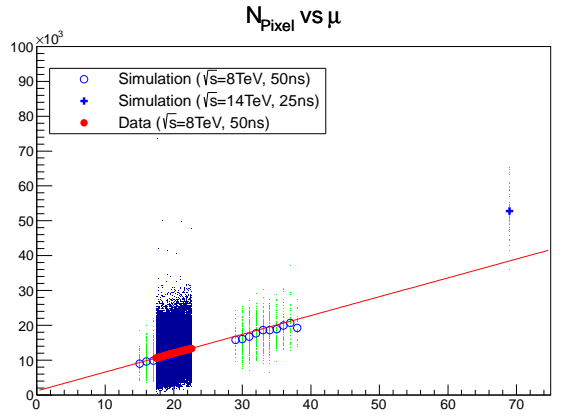

(a)

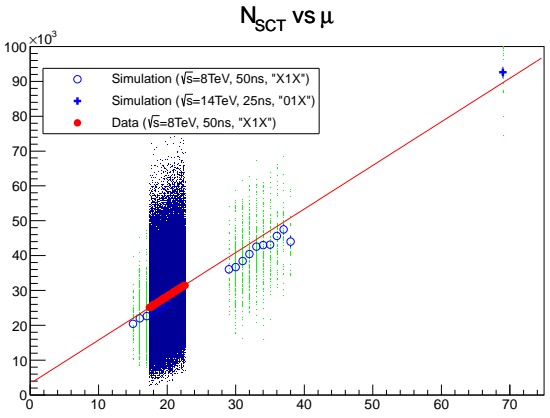

(b)

Figure 58: Number of (a) Pixel and (c) SCT hits as a function of $\langle\mu\rangle$. MC plots are superimposed to compare it with the data.

\footnotetext{
${ }^{6}$ In the MC analysis, it was not possible to emulate certain timing cuts due to technical limitations.
} 


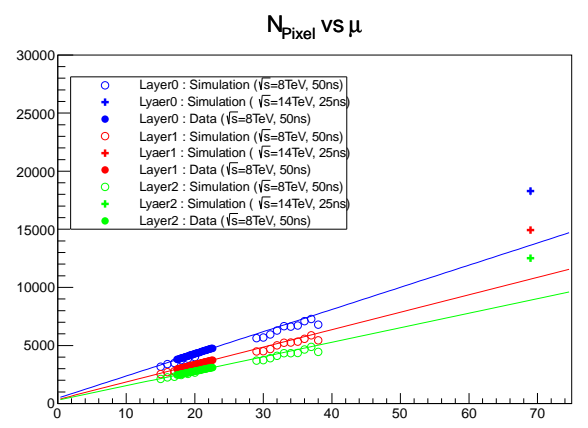

(a)

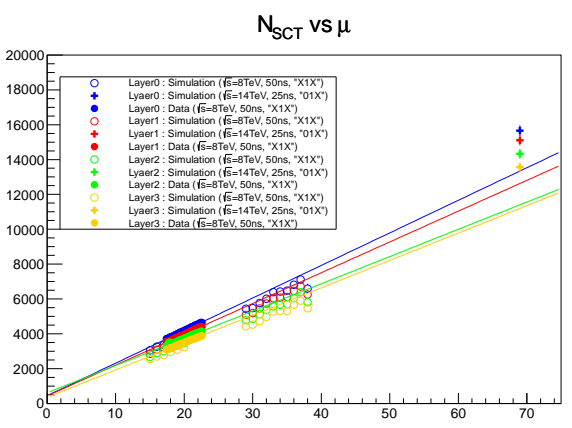

(b)

Figure 59: Number of (a) Pixel and (c) SCT hits as a function of $\langle\mu\rangle$ for individual barrel layers. MC plots are superimposed to compare it with the data. 


\section{Appendix O Clustering}

\section{O.1 Output Data Volume}

Up until this point we have assumed no input data volume reduction due to clustering. In this section we present numbers for data volume and bandwidth requirements assuming that 2D Pixel clustering reduces the hit count by a factor of 3. Likewise we assume that linear clustering algorithms reduce the number of SCT hits by a factor of 2. Table 28 and Table O.1 show output data volume $\left(N_{\text {hits }}\right)$ with the assumption of the data reduction. Note that the numbers are extrapolated into the high luminosity LHC condition of $\sqrt{s}=14 \mathrm{TeV},\langle\mu\rangle=70$, and $25 \mathrm{~ns}$ bunch spacing.

\begin{tabular}{|c||c|c|c|c||c|c|c|c|c||c|c|}
\hline Tower & P-B-L0 & P-B-L1 & P-B-L2 & P-EC & S-B-L0 & S-B-L1 & S-B-L2 & S-B-L3 & S-EC & $N_{\text {pxl }}$ & $N_{\text {SCT }}$ \\
\hline 0 & 447 & 292 & 198 & 130 & 304 & 151 & 73 & 0 & 1684 & 1068 & 2212 \\
\hline 1 & 525 & 262 & 198 & 114 & 301 & 188 & 73 & 0 & 1694 & 1099 & 2255 \\
\hline 2 & 533 & 264 & 172 & 105 & 298 & 150 & 72 & 0 & 1780 & 1073 & 2300 \\
\hline 3 & 540 & 334 & 166 & 115 & 298 & 187 & 72 & 0 & 1787 & 1156 & 2345 \\
\hline 4 & 521 & 256 & 180 & 124 & 301 & 151 & 72 & 0 & 1693 & 1081 & 2218 \\
\hline 5 & 523 & 323 & 186 & 124 & 301 & 191 & 72 & 0 & 1680 & 1156 & 2245 \\
\hline 6 & 346 & 266 & 175 & 133 & 301 & 151 & 74 & 0 & 1713 & 921 & 2239 \\
\hline 7 & 506 & 266 & 173 & 134 & 303 & 188 & 74 & 0 & 1698 & 1079 & 2263 \\
\hline 8 & 527 & 321 & 207 & 135 & 302 & 152 & 73 & 0 & 1597 & 1190 & 2125 \\
\hline 9 & 513 & 253 & 206 & 132 & 304 & 191 & 72 & 0 & 1614 & 1104 & 2181 \\
\hline 10 & 508 & 263 & 160 & 131 & 307 & 154 & 73 & 0 & 1731 & 1061 & 2265 \\
\hline 11 & 472 & 325 & 151 & 132 & 305 & 185 & 73 & 0 & 1772 & 1081 & 2336 \\
\hline 12 & 487 & 247 & 205 & 131 & 280 & 152 & 74 & 0 & 1683 & 1071 & 2189 \\
\hline 13 & 513 & 308 & 203 & 130 & 280 & 191 & 74 & 0 & 1689 & 1155 & 2235 \\
\hline 14 & 342 & 252 & 135 & 113 & 305 & 152 & 73 & 0 & 1770 & 842 & 2301 \\
\hline 15 & 444 & 245 & 144 & 121 & 306 & 188 & 73 & 0 & 1784 & 953 & 2351 \\
\hline 16 & 373 & 334 & 261 & 0 & 525 & 461 & 513 & 483 & 0 & 968 & 1982 \\
\hline 17 & 449 & 297 & 262 & 0 & 521 & 555 & 513 & 483 & 0 & 1008 & 2072 \\
\hline 18 & 453 & 299 & 229 & 0 & 491 & 459 & 513 & 483 & 0 & 981 & 1946 \\
\hline 19 & 460 & 380 & 223 & 0 & 491 & 575 & 511 & 457 & 0 & 1063 & 2034 \\
\hline 20 & 472 & 283 & 222 & 0 & 517 & 463 & 494 & 467 & 0 & 977 & 1941 \\
\hline 21 & 472 & 359 & 221 & 0 & 524 & 582 & 511 & 479 & 0 & 1052 & 2095 \\
\hline 22 & 265 & 308 & 221 & 0 & 523 & 464 & 514 & 482 & 0 & 794 & 1983 \\
\hline 23 & 373 & 305 & 221 & 0 & 517 & 586 & 513 & 485 & 0 & 899 & 2101 \\
\hline 24 & 423 & 366 & 265 & 0 & 524 & 469 & 515 & 485 & 0 & 1054 & 1993 \\
\hline 25 & 439 & 289 & 276 & 0 & 526 & 584 & 508 & 488 & 0 & 1004 & 2107 \\
\hline 26 & 432 & 300 & 212 & 0 & 528 & 464 & 509 & 484 & 0 & 944 & 1985 \\
\hline 27 & 396 & 368 & 190 & 0 & 528 & 557 & 513 & 482 & 0 & 954 & 2080 \\
\hline 28 & 413 & 280 & 248 & 0 & 500 & 443 & 515 & 472 & 0 & 942 & 1929 \\
\hline 29 & 442 & 354 & 266 & 0 & 496 & 582 & 516 & 473 & 0 & 1063 & 2066 \\
\hline 30 & 295 & 289 & 194 & 0 & 522 & 463 & 513 & 482 & 0 & 778 & 1980 \\
\hline 31 & 368 & 281 & 208 & 0 & 526 & 574 & 513 & 481 & 0 & 858 & 2095 \\
\hline \hline & & & & & & & & & & & \\
\hline
\end{tabular}

Table 28: The number of hits in the output stream from DF to AUX or SSB. The numbers extracted from a run with $\sqrt{s}=8 \mathrm{TeV}$ and $\langle\mu\rangle=30$ (Run:214523, LB:153) assuming clustering reductions and extrapolated into $14 \mathrm{TeV}$ and $\langle\mu\rangle=70$. This table summarized Tower ID 0 to 31 for C-Side. Number of SCT hits are counted with "edge mode" emulation. 


\begin{tabular}{|c|c|c|c|c|c|c|c|c|c|c|c|}
\hline Tower & P-B-L0 & P-B-L1 & P-B-L2 & P-EC & S-B-L0 & S-B-L1 & S-B-L2 & S-B-L3 & S-EC & $N_{\mathrm{pxl}}$ & $N_{\mathrm{SCT}}$ \\
\hline 32 & 348 & 321 & 250 & 0 & 489 & 433 & 483 & 448 & 0 & 919 & 1853 \\
\hline 33 & 420 & 277 & 250 & 0 & 487 & 543 & 479 & 449 & 0 & 948 & 1958 \\
\hline 34 & 422 & 279 & 215 & 0 & 485 & 432 & 476 & 449 & 0 & 916 & 1842 \\
\hline 35 & 428 & 353 & 215 & 0 & 486 & 535 & 476 & 435 & 0 & 996 & 1932 \\
\hline 36 & 439 & 262 & 218 & 0 & 487 & 429 & 475 & 434 & 0 & 919 & 1825 \\
\hline 37 & 416 & 334 & 211 & 0 & 491 & 539 & 476 & 444 & 0 & 961 & 1951 \\
\hline 38 & 224 & 286 & 205 & 0 & 492 & 432 & 479 & 447 & 0 & 715 & 1850 \\
\hline 39 & 370 & 274 & 193 & 0 & 488 & 544 & 478 & 447 & 0 & 836 & 1957 \\
\hline 40 & 417 & 332 & 235 & 0 & 488 & 432 & 478 & 450 & 0 & 984 & 1849 \\
\hline 41 & 409 & 269 & 258 & 0 & 490 & 540 & 479 & 451 & 0 & 936 & 1959 \\
\hline 42 & 381 & 278 & 202 & 0 & 492 & 432 & 480 & 449 & 0 & 861 & 1853 \\
\hline 43 & 346 & 341 & 182 & 0 & 492 & 535 & 476 & 447 & 0 & 869 & 1950 \\
\hline 44 & 385 & 261 & 232 & 0 & 490 & 426 & 475 & 435 & 0 & 877 & 1826 \\
\hline 45 & 412 & 330 & 249 & 0 & 485 & 534 & 476 & 436 & 0 & 992 & 1932 \\
\hline 46 & 274 & 270 & 181 & 0 & 487 & 427 & 474 & 434 & 0 & 726 & 1822 \\
\hline 47 & 344 & 272 & 207 & 0 & 490 & 535 & 480 & 435 & 0 & 823 & 1941 \\
\hline 48 & 441 & 299 & 186 & 122 & 283 & 141 & 68 & 0 & 1582 & 1048 & 2075 \\
\hline 49 & 498 & 244 & 186 & 116 & 283 & 178 & 68 & 0 & 1591 & 1044 & 2120 \\
\hline 50 & 503 & 247 & 161 & 124 & 282 & 141 & 68 & 0 & 1664 & 1034 & 2154 \\
\hline 51 & 509 & 310 & 160 & 116 & 282 & 174 & 67 & 0 & 1657 & 1096 & 2180 \\
\hline 52 & 520 & 248 & 181 & 117 & 284 & 140 & 67 & 0 & 1553 & 1066 & 2043 \\
\hline 53 & 496 & 310 & 181 & 125 & 285 & 175 & 68 & 0 & 1563 & 1112 & 2091 \\
\hline 54 & 249 & 249 & 155 & 115 & 284 & 139 & 67 & 0 & 1655 & 768 & 2145 \\
\hline 55 & 421 & 237 & 128 & 115 & 284 & 176 & 67 & 0 & 1671 & 902 & 2198 \\
\hline 56 & 490 & 298 & 161 & 122 & 236 & 140 & 68 & 0 & 1571 & 1072 & 2015 \\
\hline 57 & 481 & 245 & 185 & 116 & 235 & 175 & 68 & 0 & 1570 & 1027 & 2048 \\
\hline 58 & 454 & 243 & 152 & 107 & 283 & 140 & 69 & 0 & 1642 & 955 & 2134 \\
\hline 59 & 422 & 301 & 140 & 113 & 281 & 174 & 67 & 0 & 1639 & 976 & 2161 \\
\hline 60 & 461 & 230 & 173 & 113 & 282 & 139 & 67 & 0 & 1542 & 977 & 2030 \\
\hline 61 & 484 & 277 & 184 & 121 & 283 & 175 & 68 & 0 & 1569 & 1067 & 2096 \\
\hline 62 & 321 & 229 & 113 & 120 & 281 & 141 & 69 & 0 & 1667 & 782 & 2158 \\
\hline 63 & 437 & 242 & 154 & 121 & 281 & 176 & 68 & 0 & 1680 & 953 & 2206 \\
\hline
\end{tabular}

Table 29: The number of hits in the output stream from DF to AUX or SSB. The numbers are extracted from a run with $\sqrt{s}=8 \mathrm{TeV}$ and $\langle\mu\rangle=30$ (Run:214523, LB:153) assuming clustering reductions and extrapolated into $14 \mathrm{TeV}$ and $\langle\mu\rangle=70$. This table summarized Tower ID 32 to 64 for the A-Side. Number of SCT hits are counted with "edge mode" emulation. 


\section{O.2 Bandwidth Requirement}

Table 30 and Table 31 show data volume transferred in the DF system and the corresponding data bandwidth requirements, assumption clustering data reduction. Note that the numbers are extrapolated to high luminosity LHC operating conditions $\sqrt{s}=14 \mathrm{TeV},\langle\mu\rangle=70$, and 25 ns bunch spacing.

\begin{tabular}{|c|c|c|}
\hline & average of $\langle N\rangle$ & maximum of $\langle N\rangle$ \\
\hline Total (output stream) & $3.4 \mathrm{e}+03$ & $3.9 \mathrm{e}+03$ \\
\hline Pixel Total (output stream) & $1.1 \mathrm{e}+03$ & $1.3 \mathrm{e}+03$ \\
SCT Total (output stream) & $2.3 \mathrm{e}+03$ & $2.6 \mathrm{e}+03$ \\
\hline AUX & $2.6 \mathrm{e}+03$ & $2.8 \mathrm{e}+03$ \\
SSB & $8.8 \mathrm{e}+02$ & $1.0 \mathrm{e}+03$ \\
\hline Fabric & $3.8 \mathrm{e}+02$ & $1.1 \mathrm{e}+03$ \\
Local Bus & $1.5 \mathrm{e}+03$ & $2.4 \mathrm{e}+03$ \\
Inter-Crate & $8.7 \mathrm{e}+02$ & $1.9 \mathrm{e}+03$ \\
\hline
\end{tabular}

Table 30: Summary of the expected $N_{\text {hits }}$ with $\sqrt{s}=14 \mathrm{TeV},\langle\mu\rangle=70.0$, and $25 \mathrm{~ns}$ bunch spacing. These figures are extrapolated as described in Section 8.2.8. Clustering is assumed.

\begin{tabular}{|c|c|c|c||c|}
\hline & average of $\langle N\rangle$ & maximum of $\langle N\rangle$ & BW requirement & Reserved BW \\
\hline Total (output stream) & $2.6 \mathrm{e}+03$ & $2.8 \mathrm{e}+03$ & - & - \\
\hline Pixel Total (output stream) & $1.2 \mathrm{e}+03$ & $1.4 \mathrm{e}+03$ & - & - \\
SCT Total (output stream) & $1.4 \mathrm{e}+03$ & $1.5 \mathrm{e}+03$ & - & - \\
\hline AUX & $2.0 \mathrm{e}+03$ & $2.2 \mathrm{e}+03$ & 8.98 & 48 \\
SSB & $5.2 \mathrm{e}+02$ & $5.9 \mathrm{e}+02$ & 2.37 & 6 \\
\hline Fabric & $2.9 \mathrm{e}+02$ & $6.6 \mathrm{e}+02$ & 2.62 & 10 \\
Local Bus & $1.1 \mathrm{e}+03$ & $1.9 \mathrm{e}+03$ & 7.52 & 24 \\
Inter-Crate & $6.6 \mathrm{e}+02$ & $1.4 \mathrm{e}+03$ & 5.58 & 20 \\
\hline
\end{tabular}

Table 31: Summary of the expected $N_{\text {words }}$ with $\sqrt{s}=14 \mathrm{TeV},\langle\mu\rangle=70.0$, and 25 ns bunch spacing. These figures are extrapolated as described in Section 8.2.8. The third column shows the "worst case" bandwidth requirement in Gb/s. The forth column shows the reserved bandwidth (in Gb/s) for the current Data Formatter design. Clustering is assumed. 


\section{Appendix P Unconstrained Data Volume Study}

As previously mentioned the inner detector readout system was not originally designed for a track trigger. Modules were connected to RODs to minimize data rates and balance bandwidth. In this section we consider Data Formatter performance assuming an idealized moduleROD and ROD-DF mapping.

\section{P.1 Data Sharing}

Refer to Figure 15 to compare these idealized results with the "real world" module-ROD cabling constraints.

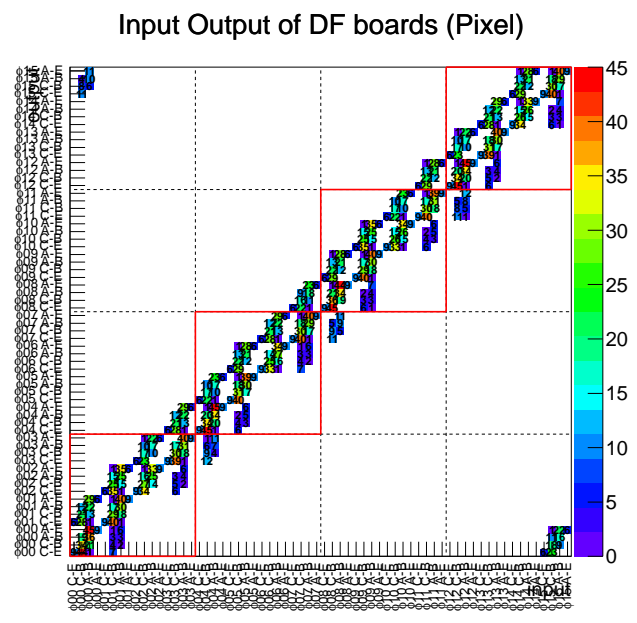

(a)

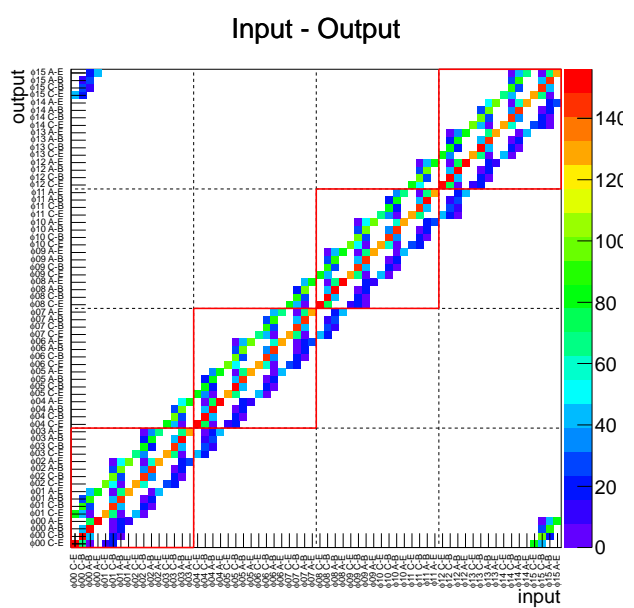

(c)

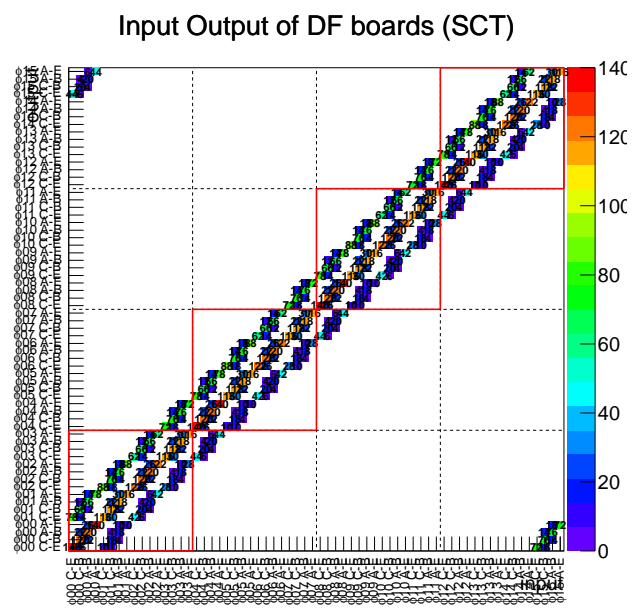

(b)

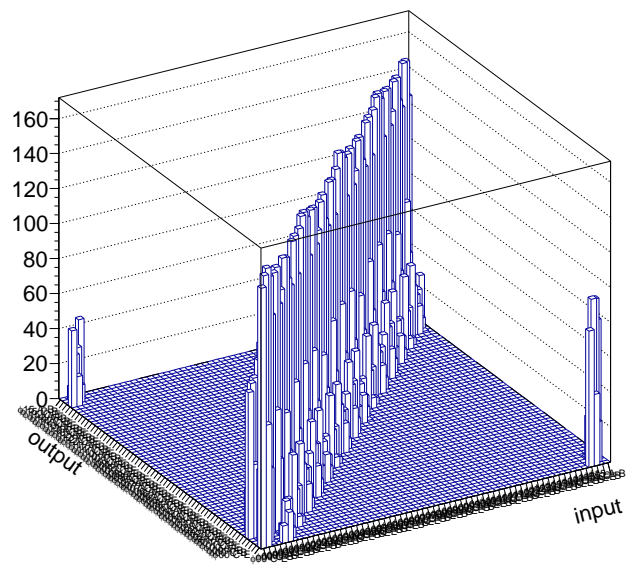

(d)

Figure 60: (a) and (b) show module sharing between two FPGAs for data from Pixel and SCT, respectively. (c) and (d) show the sum of both Pixel and SCT.

The details of the cabling can be found at http://hep.uchicago.edu/ okumura/works/ docs/20120628/idealstudy_input.xlsx. 


\section{P.2 Data Volume Study Results}

After removing the "real world" module-ROD cabling constraints we expect the data volume inside the Data Formatter system to change significantly. Figure 62 and Figure 61 show the $N_{\text {hits }}$ and $N_{\text {words }}$, respectively. These figures assume LHC operating conditions of $\sqrt{s}=8 \mathrm{TeV}$, $\langle\mu\rangle=30.0$, with a 50 ns bunch spacing. Table 32 shows the expected number of words and the required bandwidth for $\sqrt{s}=14 \mathrm{TeV},\langle\mu\rangle=70.0$, and $25 \mathrm{~ns}$ bunch spacing.

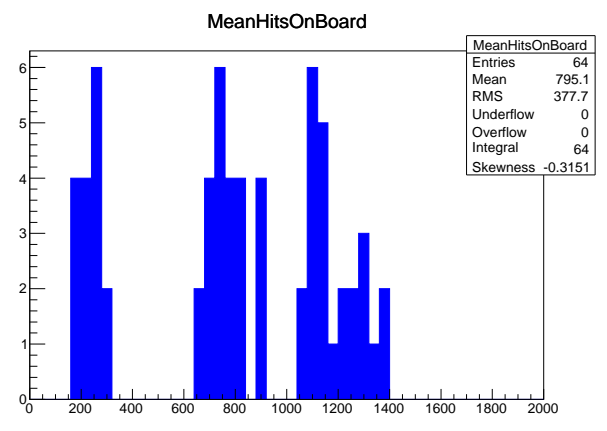

(a)

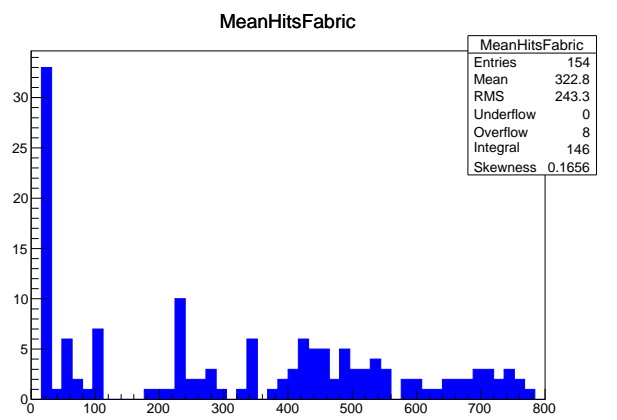

(b)

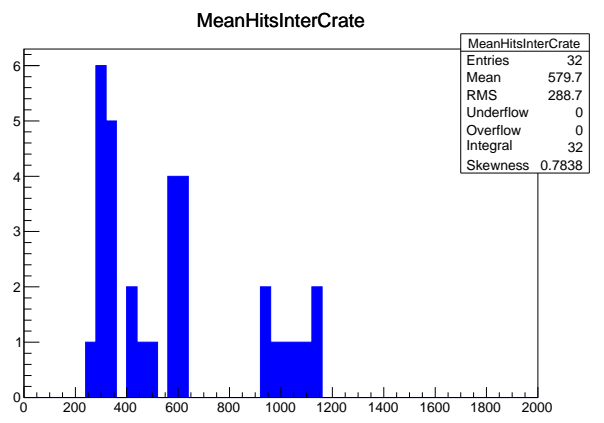

(c)

Figure 61: Summary of sum of $N_{\text {Pixel }}$ and $N_{\mathrm{SCT}}$ transferred in the DF system without module-ROD cabling constraints. These three histograms show the data volume transferred over the (a) local bus, (b) backplane Fabric Interface, and (c) the inter-shelf links. LHC operating conditions are $\sqrt{s}=8 \mathrm{TeV},\langle\mu\rangle=30.0$, with a $50 \mathrm{~ns}$ bunch spacing. Refer Figure 22 for the original study. 


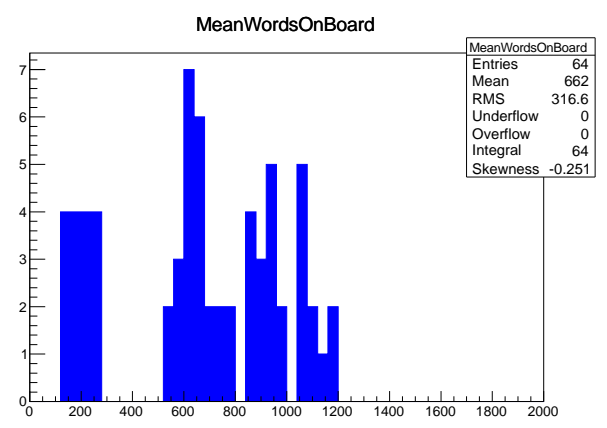

(a)

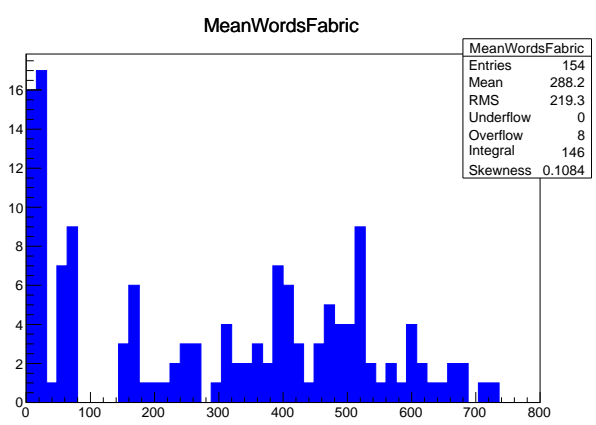

(b)

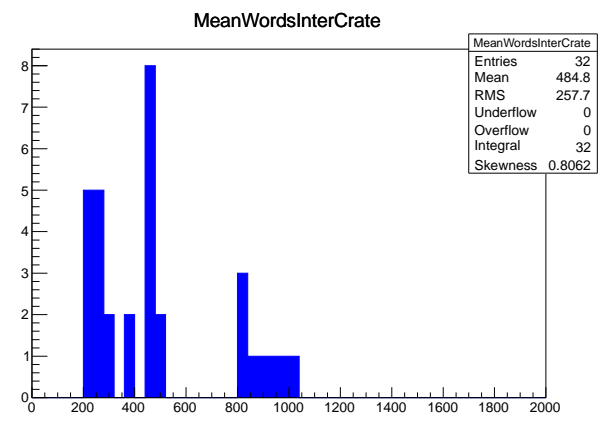

(c)

Figure 62: Summary of $N_{\text {words }}$ transferred in the Data Formatter system, assuming no moduleROD cabling constraints. LHC operating conditions are $\sqrt{s}=8 \mathrm{TeV},\langle\mu\rangle=30.0$, with a $50 \mathrm{~ns}$ bunch spacing. Refer to Figure 25 for the original study. 


\begin{tabular}{|c|c|c|c||c|}
\hline & average of $\langle N\rangle$ & maximum of $\langle N\rangle$ & BW requirement & Reserved BW \\
\hline Total (output stream) & $5.9 \mathrm{e}+03$ & $6.6 \mathrm{e}+03$ & - & - \\
\hline Pixel Total (output stream) & $3.4 \mathrm{e}+03$ & $4.0 \mathrm{e}+03$ & - & - \\
SCT Total (output stream) & $2.6 \mathrm{e}+03$ & $2.9 \mathrm{e}+03$ & - & - \\
\hline AUX & $5.0 \mathrm{e}+03$ & $5.6 \mathrm{e}+03$ & 22.5 & 48 \\
SSB & $9.6 \mathrm{e}+02$ & $1.1 \mathrm{e}+03$ & 4.3 & 10 \\
\hline Fabric & $9.7 \mathrm{e}+02$ & $2.6 \mathrm{e}+03$ & 10.5 & 10 \\
Local Bus & $2.0 \mathrm{e}+03$ & $3.6 \mathrm{e}+03$ & 14.4 & 24 \\
Inter-Crate & $1.5 \mathrm{e}+03$ & $3.2 \mathrm{e}+03$ & 12.7 & 20 \\
\hline
\end{tabular}

Table 32: Summary of the expected $N_{\text {words }}$ and bandwidth requirements with $\sqrt{s}=14 \mathrm{TeV}$, $\langle\mu\rangle=70.0$, and $25 \mathrm{~ns}$ bunch spacing with no module-ROD cabling constraints. The third column shows the "worst case" bandwidth requirement in Gb/s. The forth column shows the system bandwidth in $\mathrm{Gb} / \mathrm{s}$. 


\section{References}

[1] Data Formatter Initial Design Study and Hardware Specification (DRAFT)

J. Olsen, T. Liu, B. Penning, H.L. Li

Fermi National Accelerator Laboratory and The University of Chicago.

Fermilab Technical Publication TM-2546-PPD

[2] J. Olsen et al. "A Data Formatter for the ATLAS Fast Tracker" in IEEE Real Time Systems Symposium, Berkeley, CA, 2012.

[3] FTK: a hardware track finder for the ATLAS trigger Technical Proposal

[4] A Fast General-Purpose Clustering Algorithm

Based on FPGAs for High-Throughput Data Processing

A. Annoiv and M. Beretta

INFN - Laboratori Nazionali di Frascati, via E. Fermi 40, Frascati

[5] PICMG 3.0 AdvancedTCA Base Specification (Shortform)

http://www.picmg.org/v2internal/shortformspecs.htm

[6] PICMG 3.8 AdvancedTCA Rear Transition Module Zone 3A Specification http://www.picmg.org

[7] CERN SLINK Homepage http://hsi.web.cern.ch/hsi/s-link

[8] CERN xTCA Resources Wiki

https://twiki.cern.ch/twiki/bin/view/XTCA/WebHome

[9] FPGA Mezzanine Card Specification (VITA57.1)

http://www.vita.com/fmc.html

[10] Xilinx Kintex KC705 Development Board http://www.xilinx.com/products/boards-and-kits/EK-K7-KC705-G.htm

[11] Xilinx Development Boards http://www.xilinx.com/products/boards_kits/fmc.htm

[12] ATLAS Silicon Readout Driver (ROD) Users Manual http://www-eng.lbl.gov/ jmjoseph/Atlas-SiROD/Manuals/usersManual-v164.pdf

[13] Data Formatter FPGA Interconnections (animation) http://home.fnal.gov/ jamieson/temp/DF.mpg

[14] Xilinx Serial I/O for 7-Series FPGAs UG471 http://www.xilinx.com/support/documentation/user_guides/ug471_7Series_SelectI0. pdf

[15] Samtec VITA 57 FMC SEARAY Connectors http://www. samtec.com/search/vita57fmc.aspx

[16] Owen Boyle, Robert McLaren, Erik van der Bij, The S-LINK Interface Specification, 1997

[17] ATLAS software Pixel Cabling Service https://svnweb.cern.ch/trac/atlasoff/browser/ InnerDetector/InDetDetDescr/PixelCabling/trunk/share/Pixels_Atlas_IdMapping_ May08.dat

[18] ATLAS software SCT Cabling Service https://svnweb.cern.ch/trac/atlasoff/browser/ InnerDetector/InDetDetDescr/SCT_Cabling/trunk/share/SCT_Sept08Cabling_svc.dat 